\title{
Guidance and Visualization for Brain Tumor Surgery
}

Filipe Miguel Maria Marreiros

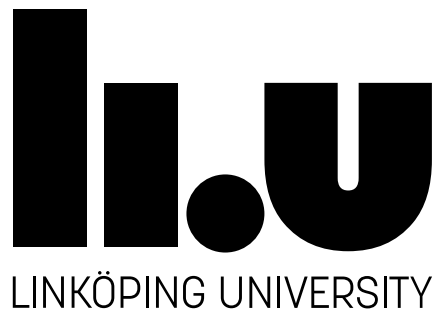

Division of Media and Information Technology (MIT) Department of Science and Technology Linköping University SE-601 74 Norrköping, Sweden

Center for Medical Image Science and Visualization (CMIV) Linköping University/US SE-581 85 Linköping, Sweden 
Copyright (c) 2016 Filipe Miguel Maria Marreiros (unless otherwise noted)

ISBN 978-91-7685-772-4

ISSN $0345-7524$

Printed in Sweden by LiU Tryck, Linköping, 2016 


\section{Abstract}

Image guidance and visualization play an important role in modern surgery to help surgeons perform their surgical procedures. Here, the focus is on neurosurgery applications, in particular brain tumor surgery where a craniotomy (opening of the skull) is performed to access directly the brain region to be treated. In this type of surgery, once the skull is opened the brain can change its shape, and this deformation is known as brain shift. Moreover, the boundaries of many types of tumors are difficult to identify by the naked eye from healthy tissue. The main goal of this work was to study and develop image guidance and visualization methods for tumor surgery in order to overcome the problems faced in this type of surgery.

Due to brain shift the magnetic resonance dataset acquired before the operation (preoperatively) no longer corresponds to the anatomy of the patient during the operation (intraoperatively). For this reason, in this work methods were studied and developed to compensate for this deformation. To guide the deformation methods, information of the superficial vessel centerlines of the brain was used. A method for accurate (approximately $1 \mathrm{~mm}$ ) reconstruction of the vessel centerlines using a multiview camera system was developed. It uses geometrical constraints, relaxation labeling, thin plate spline filtering and finally mean shift to find the correct correspondences between the camera images.

A complete non-rigid deformation pipeline was initially proposed and evaluated with an animal model. From these experiments it was observed that although the traditional non-rigid registration methods (in our case coherent point drift) were able to produce satisfactory vessel correspondences between preoperative and intraoperative vessels, in some specific areas the results were suboptimal. For this reason a new method was proposed that combined the coherent point drift and thin plate spline semilandmarks. This combination resulted in an accurate (below $1 \mathrm{~mm}$ ) non-rigid registration method, evaluated with simulated data where artificial deformations were performed.

Besides the non-rigid registration methods, a new rigid registration method to obtain the rigid transformation between the magnetic resonance dataset and the neuronavigation coordinate systems was also developed.

Once the rigid transformation and the vessel correspondences are known, 
the thin plate spline can be used to perform the brain shift deformation. To do so, we have used two approaches: a direct and an indirect. With the direct approach, an image is created that represents the deformed data, and with the indirect approach, a new volume is first constructed and only after that can the deformed image be created. A comparison of these two approaches, implemented for the graphics processing units, in terms of performance and image quality, was performed. The indirect method was superior in terms of performance if the sampling along the ray is high, in comparison to the voxel grid, while the direct was superior otherwise. The image quality analysis seemed to indicate that the direct method is superior.

Furthermore, visualization studies were performed to understand how different rendering methods and parameters influence the perception of the spatial position of enclosed objects (typical situation of a tumor enclosed in the brain). To test these methods a new single-monitor-mirror stereoscopic display was constructed. Using this display, stereo images simulating a tumor inside the brain were presented to the users with two rendering methods (illustrative rendering and simple alpha blending) and different levels of opacity. For the simple alpha blending method an optimal opacity level was found, while for the illustrative rendering method all the opacity levels used seemed to perform similarly.

In conclusion, this work developed and evaluated 3D reconstruction, registration (rigid and non-rigid) and deformation methods with the purpose of minimizing the brain shift problem. Stereoscopic perception of the spatial position of enclosed objects was also studied using different rendering methods and parameter values. 


\section{Populärvetenskaplig sammanfattning}

Bildstyrning och visualisering spelar en viktig roll i modern kirurgi för att hjälpa kirurger att utföra sina kirurgiska ingrepp. I denna avhandling ligger fokus på neurokirurgi, i synnerhet hjärntumörkirurgi där man öppnar skallen för att få direkt tillgång till hjärnan. I denna typ av operationer kan hjärnan ändra sin form när skallen öppnas. Denna deformation kallas ofta brain shift. En annan svårihet är att många typer av tumörer är svåra att med blotta ögat skilja från frisk vävnad. Det övergripande målet för detta arbete var att studera och utveckla bildstyrnings- och visualiseringsmetoder för hjärntumörkirurgi som kan bidra till att lösa problemen vid dessa operationer.

På grund av hjärnans omformning kommer magnetkamerabilder som tagits före operationen (preoperativt) inte längre att motsvarar patientens anatomi under operationen (intraoperativt). Därför har vi utvecklats metoder för att kompensera för denna deformation. För att styra omformningen av bilden användes information om mittlinjerna i hjärnans ytliga kärl. En metod utvecklades för korrekt (inom ca $1 \mathrm{~mm}$ ) rekonstruktion av kärlmittlinjer med hjälp av ett system av flera sammankopplade kameror. För att hitta motsvarande punkter mellan de olika kamerabilderna använder metoden geometriska begränsningar kombinerade met ett antal olika optimeringsmetoder.

En komplett pipeline för icke-rigid deformering utvecklades och utvärderades med en djurmodell. Där observerades att traditionella metoder för ickerigid registrering (i vårt fall coherent point drift) kunde ge tillfredsställande överensstämmelse mellan kärlen preoperativt och intraoperativt, men att i vissa specifika områden var resultaten inte optimala. Därfor utvecklade vi en ny metod som kombinerar coherent point drift och thin plate spline semilandmarks. Med denna kombination uppnådded korrekt (under $1 \mathrm{~mm}$ ) icke-rigid registrering i en utvärdering där simulerade uppgifter utfördes med artificiella deformationer.

Förutom icke-rigid registringsmetoder utvecklades en ny metod för att uppskatta och korrigera rigida (stelkropps-) rörelser mellan magnetkamerabilderna och det koordinatsystem som används under operationen. 
När den rigida transformation och korrespondensen mellan kärlen väl är kända, kan man använda thin plate splines för att korrigera för hela deformationen. Vi har använt två metoder: en direkt och en indirekt. Den direkta metoden skapar direkt en bild med korrigerad geometri. I den indirekta metoden konstrueras först en ny volym, och först därefter skapas den deformerade bilden. Dessa två angreppssätt implementerades med grafikprocessorer och jämfördes med avseende på hastighet och bildkvalitet. Den indirekta metoden är snabbare om man följer varje stråle med små steg i relation till voxelstorleken, medan den direkta är snabbare annars. Analys av bildkvaliteten talar för att den direkta metoden är överlägsen.

Särskilda studier gjordes för att förstå hur olika renderingsmetoder och -parametrar påverkar uppfattningen av den inbördes positionen hos slutna objekt (en typisk situation är en tumör innesluten i hjärnan). För att testa dessa metoder konstruerades en ny stereoskopisk display bestående av en monitor och en spegel. Med hjälp av denna display presenterades stereobilder som simulerade en tumör i hjärnan för försökspersoner med två olika renderingsmetoder (illustrativ rendering och enkel alfablandning) och olika nivåer av opacitet. För enkel alfablandning fann man en optimal opacitetsnivå, medan illustrativ rendering gav ungefär samma resultat för alla opacitetsnivåer som testades.

Sammanfattningsvis har i detta arbete omfattat utveckling och utvärdering av metoder för 3D rekonstruktion, registrering (rigid och icke-rigid) och deformationsmetoder med syftet att minimera brain shift-problemet. Stereoskopisk uppfattning av den rumsliga placeringen hos slutna föremål studerades också med olika renderingsmetoder och parametervärden. 


\section{Acknowledgements}

I would like to acknowledge the financial support from the Visualization programme of the Swedish Foundation for Strategic Research, the KK Foundation, Vinnova, Invest in Sweden Agency, Vårdalstiftelsen (grant 2009/0079) and the Swedish Childhood Cancer Foundation (grant MT2013-0036).

This work would have not been possible without the contribution of individuals and organizations from our region (Östergötland). These truly multidisciplinary contributions involved engineering and clinical personnel in an extremely effective synergy. This has been facilitated to a great extent by the setup of our Center for Medical Image Science and Visualization (CMIV) at Linköping University, where engineers like myself share the same space with clinical personnel.

First of all, I am grateful to my supervisor Örjan Smedby for the opportunity to integrate the project that produced the papers presented in this work, and for being deeply involved in the writing of the papers, mentoring and allowing me to grow as a scientist.

To my co-supervisors Sandro Rossitti, Karin Wårdel and Anders Ynnerman, for their insights. In particular Sandro for his active participation in most of our papers. Special thanks also to the remaining co-authors and contributors, Chunliang Wang, Per Karlsson, Torbjörn Gustafsson, Per Carleberg and Johan Kihlberg. Chunliang Wang, now at the Royal Institute of Technology in Stockholm (KTH), was my officemate for many years, and during this time we had many interesting discussions which resulted in several methods like our vessel non-rigid registration method. Thank you for your positive attitude and friendship. Per M. Karlsson from the neurosurgery department of the Linköping Hospital enabled us to acquire data in the OR and participated actively in resolving practical research issues. Torbjörn Gustafsson and Per Carleberg from XM Reality AB aided me in the conceptualization and construction of our tracking system. Finally, Johan Kihlberg from CMIV actively participated in the scanning of the animals and patients, proposing MRI protocols adequate to our needs. To all, thank you for making this work possible.

To my colleagues and teachers at CMIV, Department of Biomedical Engineering (IMT), and Media and Information Technology (MIT) in Norrköping.

To Neda Haj-Hosseini for reviewing parts of the "kappa", in particular 
the section regarding fluorescence.

To Örjan Smedby and Fanny Lagerström for reviewing the "Populärvetenskaplig sammanfattning".

To my mentors and colleagues over the years, in the University of Algarve - J. M. Hans du Buf, University of Minho - Victor Alves and Adérito Marcos (now at Universidade Aberta, Lisbon), Innsbruck Medical University - Wolfgang Freysinger, Wolfgang Recheis, Michael Verius and Yann Heuzé (now at the University of Bordeaux). In fact my first contact with the methods that were used in this work, in particular Radial Basis Functions, was in the Vision Laboratory in the University of Algarve. Later, at Innsbruck Medical University, integrated in the EVAN project I was lucky to meet personally Fred Bookstein and Philipp Gunz, authors of some of the most cited papers in the subject.

To my Linköping friends Olivier Cross, Rafael Sanchez, Antonio Pinheiro, Sandra Cabral, and many others (the list is too long) that shared my life during the last few years. So many good memories guys, thank you all.

Last but not least, I would like to thank my family (Swedish and Portuguese) for all the help and support, I love you all. Flora I am so sorry I missed your birth because I was on the other side of the world at a conference but your mum did a great job. Thank you Fanny for all, my love. 


\section{List of Publications}

This dissertation is based on the following papers, which will be referred to in the text by their Roman numerals. The papers are appended at the end of the dissertation.

I F. M. M. Marreiros, S. Rossitti, P. M. Karlsson, C. Wang, T. Gustafsson, P. Carleberg and Ö. Smedby. Superficial vessel reconstruction with a multiview camera system. Journal of Medical Imaging, 3(1), 2016.

II F. M. M. Marreiros, S. Rossitti, C. Wang and Ö. Smedby. Non-rigid Deformation Pipeline for Compensation of Superficial Brain Shift. In the $16^{\text {th }}$ International Conference on Medical Image Computing and Computer Assisted Intervention (MICCAI), 16(Pt 2):141-148, 2013

III F. M. M. Marreiros, C. Wang, S. Rossitti and Ö. Smedby. Non-rigid point set registration of curves: registration of the superficial vessel centerlines of the brain. In Proceedings SPIE 9786, Medical Imaging 2016: Image-Guided Procedures, Robotic Interventions, and Modeling, 2016.

IV F. M. M. Marreiros, P. M. Karlsson, J. Kihlberg, S. Rossitti and Ö. Smedby. Rigid registration for image-guided neurosurgery optimized by the thin plate spline bending energy. Submitted for journal publication, 2016.

V F. M. M. Marreiros and Ö. Smedby. GPU-based ray-casting of non-rigid deformations: a comparison between direct and indirect approaches. In proceeding of SIGRAD 2014, :67-74, 2014.

VI F. M. M. Marreiros and Ö. Smedby. Single-Monitor-Mirror Stereoscopic Display. Journal of Graphics Tools, 17:85-97, 2015.

VII F. M. M. Marreiros and Ö. Smedby. Stereoscopic Static Depth Perception of Enclosed 3D Objects. In proceedings of the ACM Symposium on Applied Perception (SAP), :15-22, 2013. 
In addition, the following papers were published as part of the work related to this dissertation.

- F. M. M. Marreiros and Ö. Smedby. Mirror Stereoscopic Display for Direct Volume Rendering. In proceeding of SIGRAD 2014, :75-82, 2014.

- F. M. M. Marreiros, S. Rossitti, T. Gustafsson, P. Carleberg and Ö. Smedby. Multi-view 3D vessel tracking using near-infrared cameras. Journal of Computer Assisted Radiology and Surgery, 8 (Suppl. 1), Image Processing and Visualization, :165, 2013.

Scientific paper not part of the dissertation project, but related to the dissertation subject.

- F. M. M. Marreiros, Y. Heuzé, M. Verius, C. Unterhofer, W. Freysinger and W. Recheis. Custom implant design for large cranial defects. Journal of Computer Assisted Radiology and Surgery, 2016. 


\section{Contents}

1 Introduction 1

2 Background 4

2.1 Medical Imaging for Brain Tumor Surgery . . . . . . . . . . . 4

2.1.1 Coordinate Systems . . . . . . . . . . . 6

2.2 3D Image Processing and Analysis Methods . . . . . . . . . . 7

2.2.1 Segmentation . . . . . . . . . . . . 7

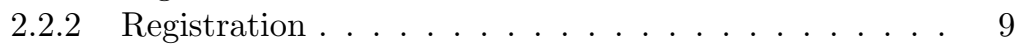

2.2 .3 Deformation . . . . . . . . . . . . . . . 12

2.2.4 Visualization ................ 12

2.3 Image-Guided Navigation Systems for Brain Tumor Surgery • 14

2.3.1 Intraoperative MRI . . . . . . . . . . . . . 15

2.3.2 Intraoperative CT . . . . . . . . . . . . . 15

2.3.3 Intraoperative Ultrasound . . . . . . . . . . . . . . . 15

2.3.4 Optical .................... . . 16

2.4 Stereoscopy and Depth Perception of Semi-Transparent Objects 23

$\begin{array}{llr}3 & \text { Aims } & 27\end{array}$

4 Summary of the Papers $\quad \mathbf{2 8}$

4.1 3D Vessel Centerline Reconstruction . . . . . . . . . . . . . 28

4.2 Brain Shift Compensation . . . . . . . . . . . . . . 29

4.3 Visualization of Enclosed Objects . . . . . . . . . . . . 35

5 Discussion $\quad 39$

5.1 Optical 3D Reconstruction . . . . . . . . . . . . . . 39

5.2 Brain Shift Compensation . . . . . . . . . . . . . 40

5.3 Visualization for Brain Tumor Surgery . . . . . . . . . . . 44

5.4 Reflections.................. . 46

6 Conclusions $\quad 48$

$\begin{array}{ll}\text { Bibliography } & 51\end{array}$ 


\section{Chapter 1}

\section{Introduction}

A brain tumor is a serious health condition, which is characterized by an abnormal growth of tissue. There are over 120 types of brain tumors, and most medical institutions use the World Health Organization (WHO) classification system [133] to identify them. The tumors can be classified as malignant or benign and according to wheather or not they contain cancer cells. Although a benign tumor does not contain cancer cells, it can still be life-threatening because it may cause pressure in certain vital regions of the brain and it may become malignant. Furthermore, the malignancy of the tumors is graded from I to IV with IV being the most malignant grade which grows and spreads faster than the lower grades.

The most current data from the WHO for Europe is from 2012, presented in Figures 1.1 and 1.2. We can observe that Sweden is one of the countries with the highest incidence of cancer of the brain and central nervous system, more than ten persons per one hundred thousand. Cancer of the brain and central nervous system is not the most incident, but it is still rather high in the list of most incident cancers, in Europe it ranks $16^{\text {th }}$ and in Sweden it is more incident ranking eighth.

In brain tumor surgery the surgeon tries to remove as much tumor as possible. If the surgeon takes too little a fast recurrence of the tumor occurs, if he takes too much the patient can be permanently disabled.

In surgeries where craniotomy is performed to access directly the brain region to be treated, the surgeon can view directly the brain surface, but the boundaries of many tumors are difficult to differentiate from healthy tissue with the naked eye. Furthermore, if the tumor is enclosed in the brain, the surgeon cannot directly identify the tumor location.

Image guidance and visualization techniques have been developed to aid in the location of regions of interest, e.g. the tumor. The accuracy of guidance tools is crucial and has a direct clinical outcome. For the highly malignant brain tumor, glioblastoma multiforme, Lacroix et al. [120] and Stummer et al. [191] reported that patients with more complete resections 
have significant survival advantages.

During surgery the brain shape can change, and this deformation is named brain shift, consequently brain shift compensation must be performed on the medical images generated prior to surgery. Several groups measured the brain shift before resection, reporting shifts of 1-2.5 cm [170, 152, 147, $85]$.

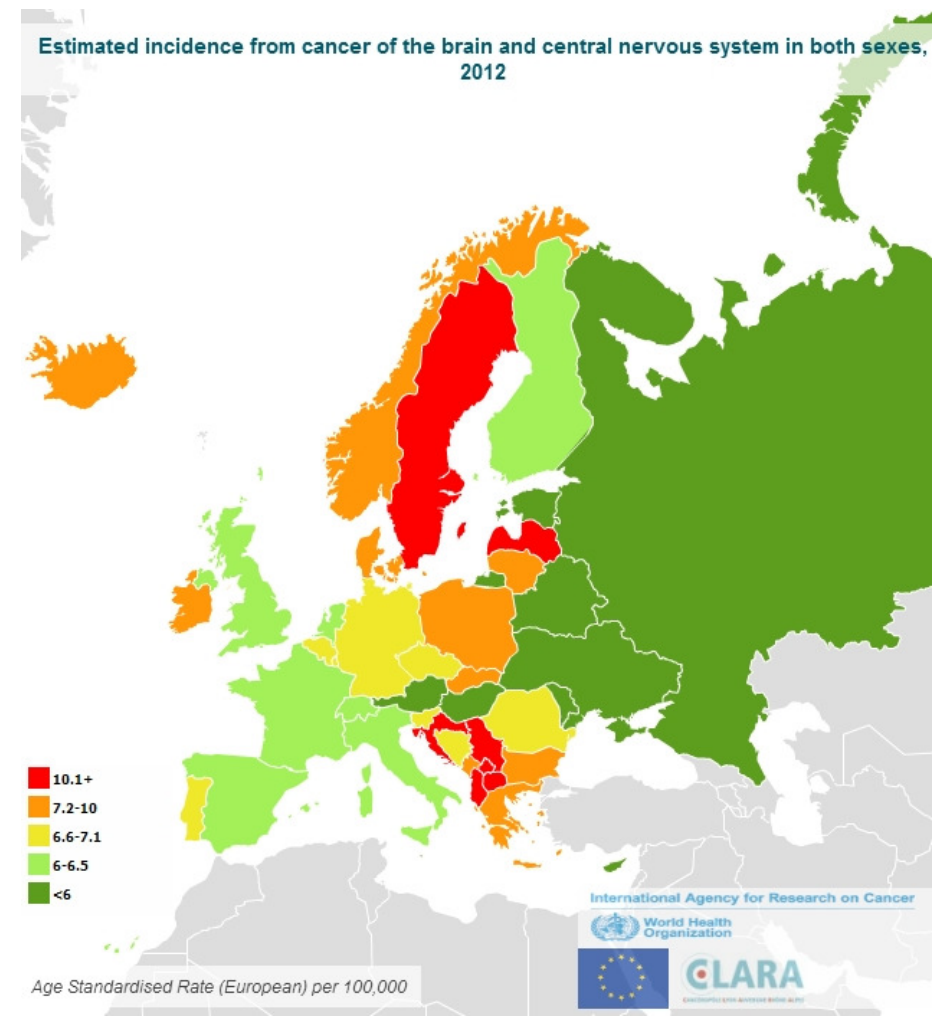

Figure 1.1: European estimated incidence from cancer of the brain and central nervous system in both sexes, 2012. 


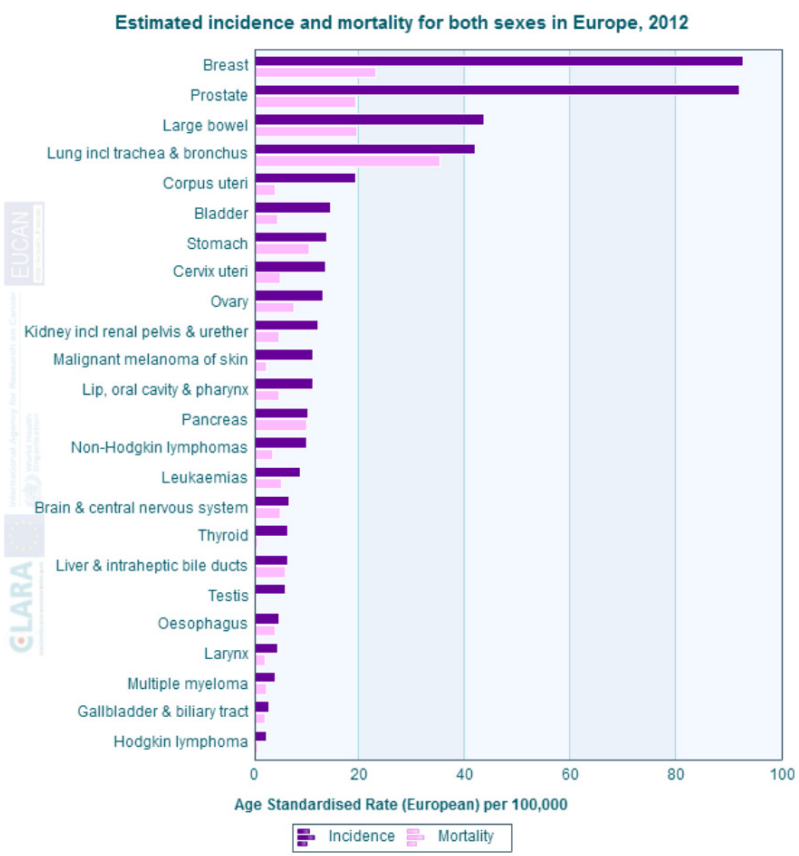

Estimated incidence and mortality for both sexes in Sweden, 2012

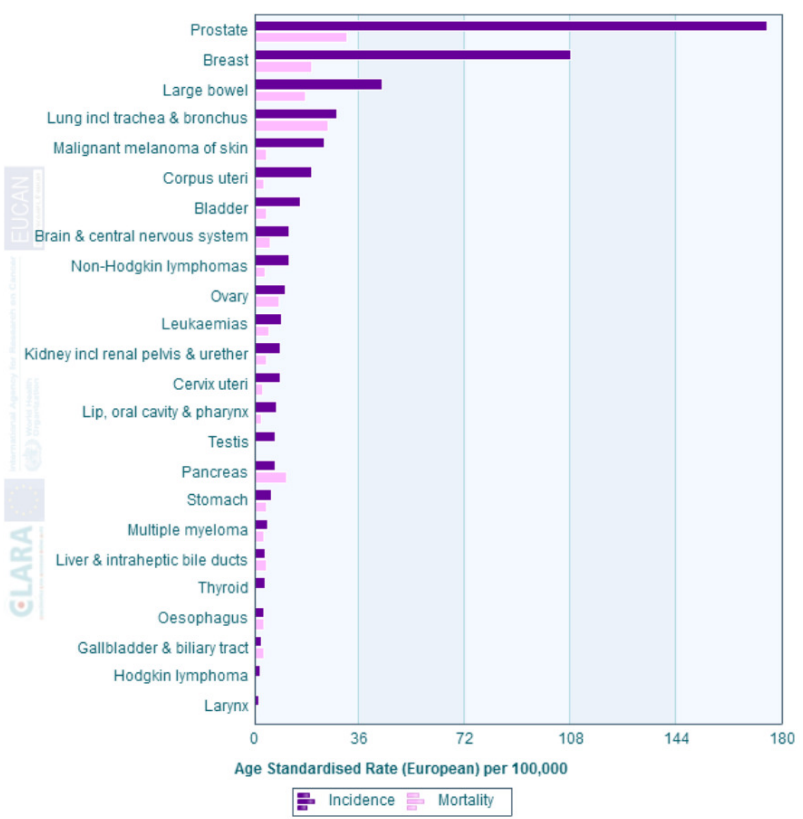

Figure 1.2: Cancer estimated incidence and mortality for both sexes in Europe (top) and Sweden (bottom), 2012. 


\section{Chapter 2}

\section{Background}

\subsection{Medical Imaging for Brain Tumor Surgery}

Imaging is routinely used for diagnosis and therapy. In the case of brain tumors, the two most important image modalities are: Computed Tomography (CT) and Magnetic Resonance Imaging (MRI) [49]. MRI is often preferred due to its better distinction of soft tissues. Both CT and MRI can use contrast agents to obtain a better delineation of the tumor.

In some cases it is necessary to investigate the metabolic or biochemical activity of the body using Positron Emission Tomography (PET) or Magnetic Resonance Spectroscopy (MRS). PET is commonly combined with CT known as PET-CT. Furthermore, the neural activity can be investigated by detecting changes of the blood flow using Functional Magnetic Resonance Imaging (fMRI) or Single-Photon Emission Computed Tomography (SPECT). The fMRI technique is mainly used to detect eloquent areas [73], i.e. areas that the surgeon should avoid, such as areas of motor function, sensitive function, speech, vision, reading, hearing, memory and learning. Tumors can also be visible using ultrasound during surgery (intraoperatively).

When an examination is performed with these modalities typically the Digital Imaging and Communications in Medicine (DICOM) [34] ${ }^{1}$ format is used and the data is stored in a Picture Archiving and Communication System (PACS). Besides specifying the file format, DICOM also specifies the communication standard. The file format typically contains descriptive information (DICOM tags) and the image raw data. These tags are of particular interest because by using some of them the spatial position of the voxels can be calculated.

In this work two MRI techniques are mainly used: T1-Weighted 3D

\footnotetext{
${ }^{1}$ http://dicom.nema.org/
} 


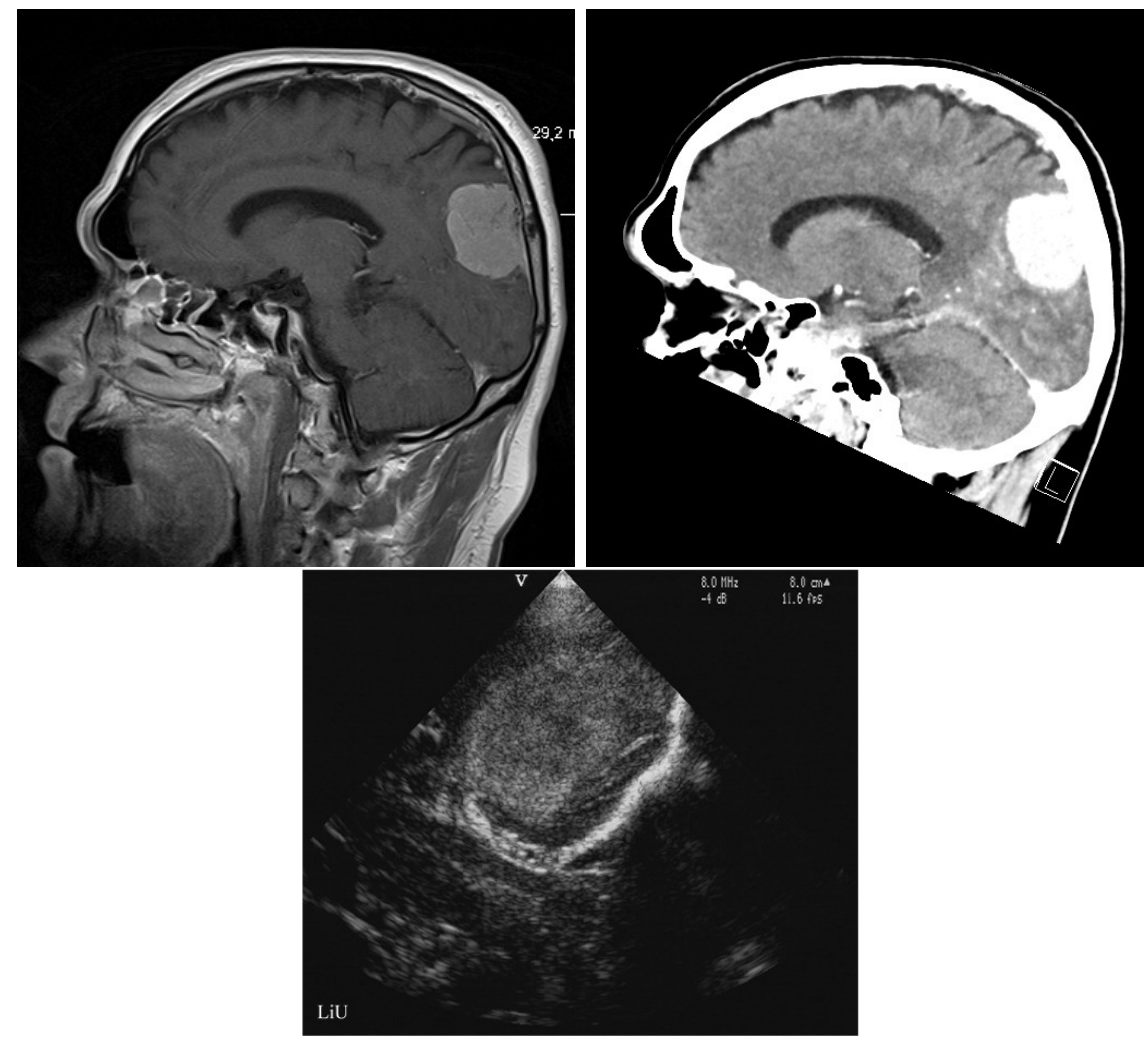

Figure 2.1: Brain tumors visible with T1W MRI(top, left), CT (top, right) and ultrasound (bottom). The $\mathrm{T} 1 \mathrm{~W}$ and $\mathrm{CT}$ datasets are from the same patient and were contrast enhanced in order to improve the visibility of the tumor. (CT and T1W images Courtesy of Inger Eweman, MD, Department of Radiology, Linköping University Hospital, Linköping, Sweden). (Ultrasound image Courtesy of Johan Richter, MD, Department of Neurosurgery, Linköping University, Linköping, Sweden).

Turbo Field Echo (T1W 3D TFE) and Phase Contrast Angiography (PCA) which is a Magnetic Resonance Angiography (MRA) technique. T1W 3D TFE is used to obtain the anatomical structures of the head, and PCA is used to obtain the vessels of the head. With the PCA technique two aligned datasets are generated, the first contains a poor representation of the anatomy and the second encodes flows with a certain velocity range, in our case the brain vessel flows, providing a representation of the vessels. In Figure 2.1 are presented images of patients with brain tumors visible with T1W MRI, CT and ultrasound.

Despite the fact that each modality produces rather different data values, depending on the tissue or function we aim to investigate, the structure of 
the data is similar (except for ultrasound). It consists of a stack of images that can be combined to form a volume. This sort of spatial data structure is named regular grid or uniform grid. A data element of the $3 \mathrm{D}$ grid is referred to as a voxel (Figure 2.2), analogous to a pixel for a $2 \mathrm{D}$ image. The voxel is typically associated with one scalar value.

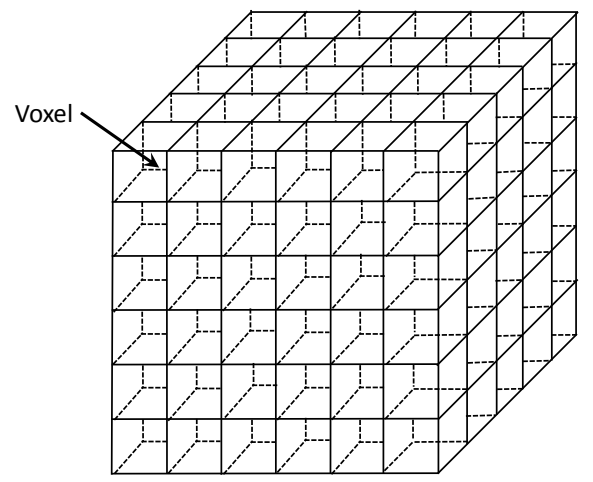

Figure 2.2: $3 \mathrm{D}$ regular grid composed of voxels.

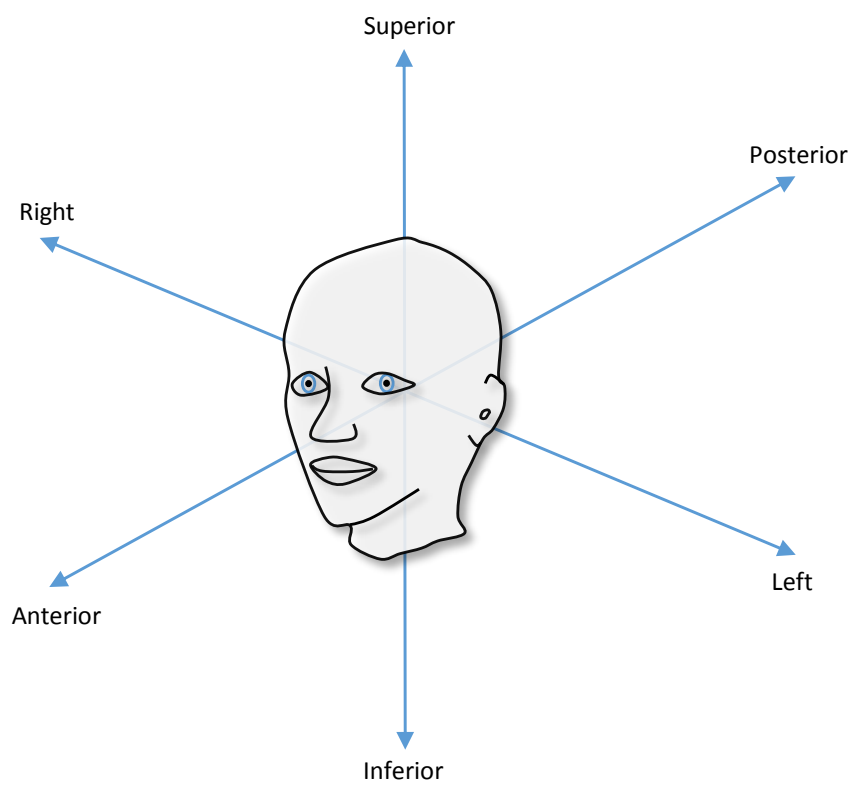

Figure 2.3: Head orientation reference. 


\subsubsection{Coordinate Systems}

For the medical datasets of the head there is a commonly used orientation reference (Right, Left, Anterior, Posterior, Inferior and Superior) as presented in Figure 2.3. A common source of mistakes is the misinterpretation of the Left and Right directions, given in relation to the patient's head. From these directions, axes for the spatial coordinate system are specified. There are two main conventions, the Neurological (Right, Anterior, Superior) and the Radiological (Left, Anterior, Superior) as presented in Figure 2.4. The direction of the axes is important because it determines whether the coordinate system is right- or left-handed. In our case, since the image coordinate system must be aligned (rigid registration) with the coordinate system of our camera system (OpenCV [20] coordinate system) then they must agree or else the alignment is impossible. The MRI datasets used in this work are mainly in DICOM format. This, specifies a Reference Coordinate System (RCS) where the $\mathrm{X}$ direction is from right to left, $\mathrm{Y}$ is from anterior to posterior, and $\mathrm{Z}$ from inferior to superior, as presented in Figure 2.5. In the same figure the OpenCV coordinate system is also presented. Both the DICOM coordinate system and the OpenCV coordinate systems are right-handed coordinate systems.

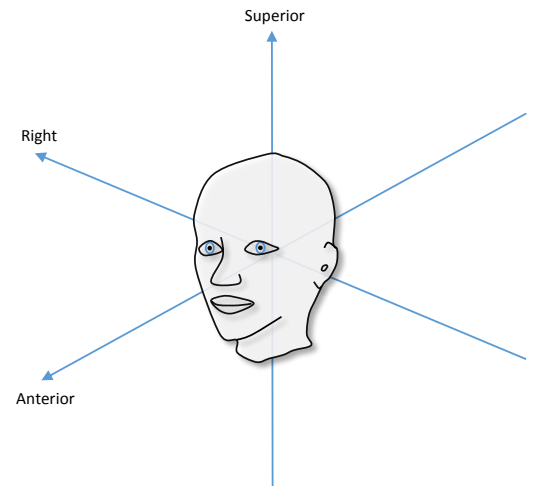

Neurological (Right, Anterior, Superior)

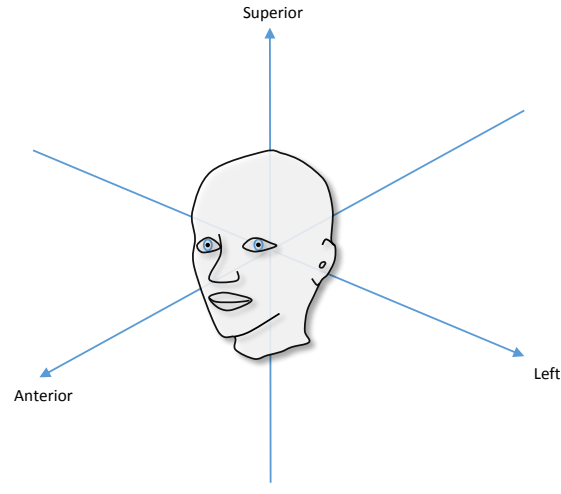

Radiological (Left, Anterior, Superior

Figure 2.4: Coordinate systems for the Neurological and Radiological conventions. 

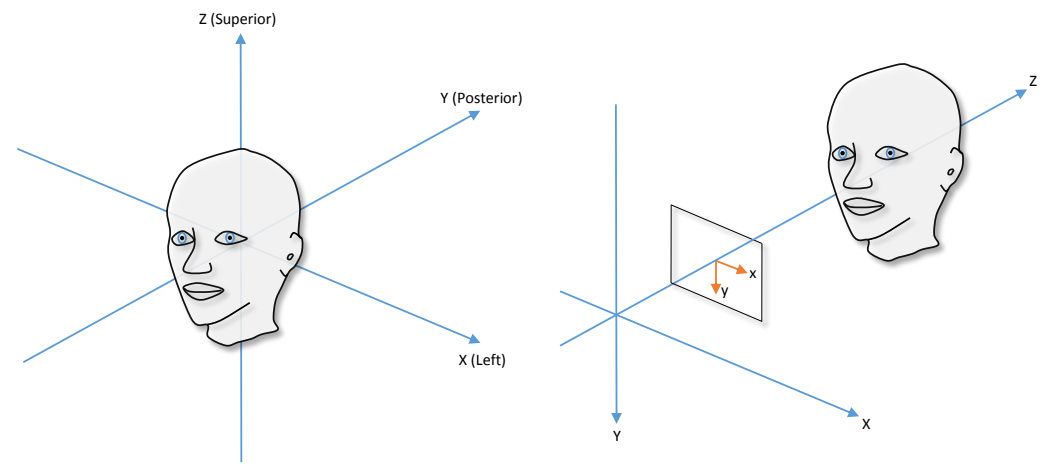

Figure 2.5: DICOM Reference Coordinate System (left) and OpenCV coordinate system (right). In the right it is also presented the OpenCV camera image plane (rectangle) and its coordinate system.

\subsection{D Image Processing and Analysis Methods}

\subsubsection{Segmentation}

In image processing, segmentation refers to the process of partitioning an image into different segments. In medical imaging, particularly in $3 \mathrm{D}$ where a volume is used, the segments often correspond to different organs, pathologies, eloquent areas, vessels and other relevant biological structures. There are innumerable segmentation techniques using different strategies, depending on the data and prior knowledge, among other factors. Good surveys can be found in $[206,54,186]$.

In this work, segmentation is mostly performed using the level set method [175], in particular the implementations by Wang et al. [212, 213] to segment the head, brain and regions of interest of the brain. These methods use seed regions that are placed on the volume and threshold values for the voxels of interest are specified. The methods iteratively propagate a contour until convergence, i.e. the contour difference between iterations is small or null. An interesting property of the level set method is that it allows splitting and merging of contours without additional functions. This makes it possible to segment multiple organs with similar properties in one pass or for example both kidneys. The result of the segmentation is typically provided either in the form of a polygon mesh (the contour surface) or by a binary volume mask ( 0 - outside the contour, 1 - inside the contour). In the case of a volume mask, different objects can be segmented and each assigned a different label for distinction. The resulting polygon mesh of a brain segmentation using the level set method [213] and a T1W 3D TFE MRI, is 
presented in Figure 2.6. The 3D MRA vessels can also be segmented using a PCA dataset and the levelset method [212]; moreover, the centerlines of the vessels can also be obtained. Besides the levelset method a large number of vessel segmentation and centerline extraction methods have been proposed for $3 \mathrm{D}$ and $2 \mathrm{D}$. The most popular rely on the information of the Hessian matrix, typically named vesselness (vessel enhancement) filters $[173,71]$ combined with thinning algorithms for centerline extraction [124]. We do not go into detail about these problems; here are just referenced some recent methods for vessel enhancement by Xiao et al.[222], Yang et al. [225], and Moreno et al. [144, 145] and for centerline extraction by Kumar et al. $[117,118]$, and Sironi et al. [184].

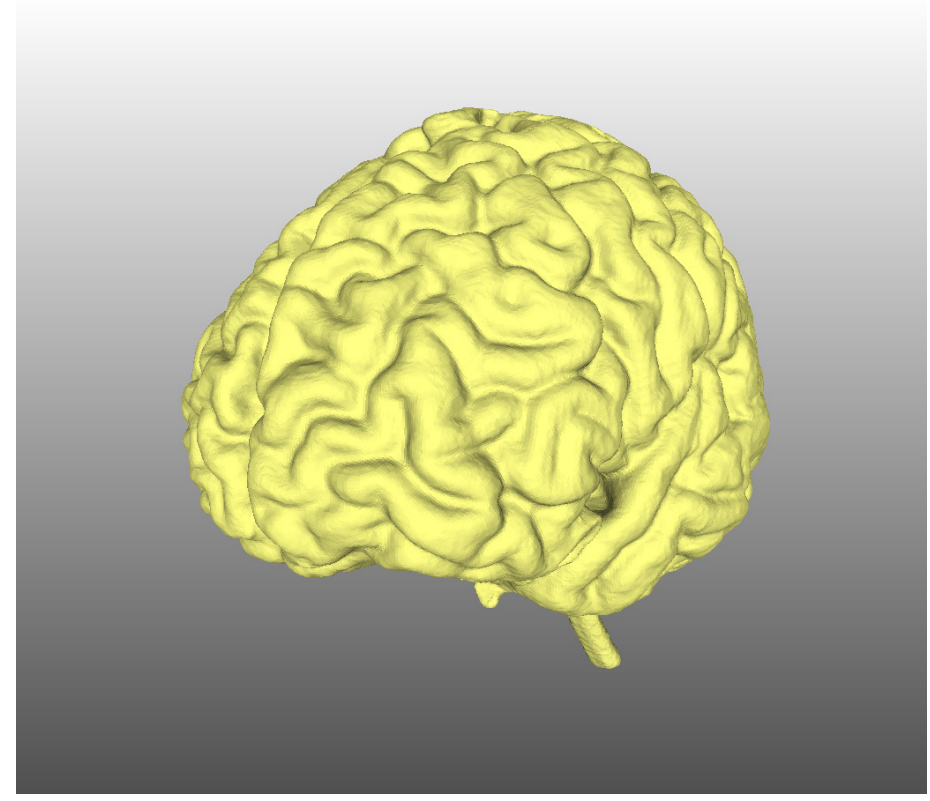

Figure 2.6: Segmented brain.

\subsubsection{Registration}

Registration is a process that searches for the correct alignment of data sets. Typically there exists a source and a target set. The registration process tries to align the sets by moving the source. Once the sets are registered, the transformation necessary to align them can be obtained.

There are many registration algorithms but two main classes exist, intensity-based registration and feature-based registration. Intensity-based registration was not used in this work because this would require information from two stacks of images (preoperative and intraoperative MRI volumes). 
This would require the use of intraoperative MRI, not used in this work. Good surveys covering most of these registration types in medical imaging can be found in $[135,206,4,154]$.

The registration used in this work is point set registration, also known as point matching, a particular type of feature-based registration. With this type of registration, point correspondences are searched to align the point sets. The registration type can also be distinguished by the transformations used, typically in an iterative optimization process. These can be of two types: rigid or non-rigid.

\section{Rigid registration}

In rigid registration the transformations preserve the distances between the points (both source and target geometries are preserved). Only rotations and translations (rigid-body transformations) are allowed.

The registration method used depends if the point correspondences are known. The first method to solve the rigid registration knowing the correspondences (homologous points) was proposed by Schönemann [174], and since then many variations of this method were proposed. In this work a Singular Value Decomposition (SVD) variant presented in [7] is used. If the correspondences are not known then e.g. the Iterative Closest Point (ICP) method $[30,15,227]$ or one of its variants $[172,12,160]$, is used.

\section{Non-rigid registration}

In non-rigid registration the source points do not need to preserve their geometry, particularly if the target geometry is different from the source. Nonrigid transformations can include affine transformations, but in the context of point set registration, they typically involve a nonlinear transformation.

Two non-rigid registration methods have gained considerable attention, the Coherent Point Drift (CPD) [146] and the Thin Plate Spline Robust Point Matching (TPS-RPM) [31, 224]. Both methods produce similar results, and a comparison can be found in [146]. In this work the CPD was extensively used. The method considers the alignment of two point sets as a probability density estimation problem, where one point set represents the Gaussian Mixture Model (GMM) centroids, and the other one represents the data points. The core of the method is to force GMM centroids to move coherently as a group to preserve the topological structure of the point sets.

If correspondences (homologous points) are available it is also possible to relax the point positions to improve the accuracy of the transformation. One such technique named: Thin Plate Spline (TPS) semilandmarks, was introduced in the context of geometric morphometrics in anthropology [185]. This technique allows the comparison of shapes between specimens. It was originally proposed by Bookstein [17] for 2D data and later extended to 3D by Gunz et al. [75, 74]. Semilandmarks are points in one of the point sets, which are free to slide from their initial position to maximize the homology 
between the shapes represented by the points. A semilandmark is able to slide along a line (on a curve), a plane (on a surface) or freely in 3D space. Tangent vectors are used to define the lines (one tangent) and planes (two tangents). An acceleration technique to speed up these calculations was proposed by Halazonetis [83].

\section{Error measures}

In order to evaluate or predict registration errors, different measures have been proposed. The three most common, proposed by Maurer et al. [105, 106] are:

- Fiducial Localization Error (FLE), which is the error in localizing the fiducial points

- Fiducial Registration Error (FRE), which is the error between homologous fiducial points after registration

- Target Registration Error (TRE), which is the error of homologous points, other than the fiducial points, after registration

A fiducial is a marker (homologous point) that is visible in both source and target datasets. Point set registration uses these points to perform the registration.

The FRE uses the Root Mean Square Error (RMSE) of the point distances to provide an overall value. For the TRE some authors use the RMSE of the point distances while others use the mean error (ME). Chai and Draxler [25] present a comparison of both measures. Here both ME and RMSE are typically presented. Fitzpatrick et al. [67, 69, 68, 66] reported that the FRE and TRE are uncorrelated but it is possible to predict the TRE using the FLE, in rigid registration. Furthermore, Shamir et al. [176, 177] studied how to optimize the rigid fiducial positions in order to minimize the TRE. The FLE is a measure of the error to localize the fiducials both in an image and in the physical space, but typically only the errors in the physical space are considered. Recently, a method to estimate the errors in the image space in CT images has been proposed [11].

\subsubsection{Deformation}

The deformation methods are directly related to the registration methods, because they can use the correspondences obtained by the registration to drive the deformation. In some cases there is no clear distinction between deformation and registration methods. The deformations methods are typically non-rigid and many have been proposed. They can be separated into physically- and non-physically-based deformation models. A good survey of physically-based methods can be found in [151]. Most of these methods 
are dependent on a mesh representation, but a subset of mesh-free methods exists.

In this work we use a non-physically-based mesh-free method, the Radial Basis Function (RBF), and one specific RBF: the TPS. It should be noted that the TPS can be used in different contexts such as, surface reconstruction, registration, and deformation, among others and it was used extensively in this work. The TPS for modeling of biological shape change, was proposed by Bookstein [18]. Bookstein formulated the TPS algebra in 2D; later extensions by Lapeer and Prager [121] enabled the use in 3D. A comparison of some of these point-based methods can be found in Fang et al. [58].

In our specific case we only have one preoperative MRI volume and two sets of points (source and target). The correspondences between the sets are obtained in the registration stage. Once these are known then the TPS can be used to construct a new volume (deformed volume - intraoperative virtual MRI).

\subsubsection{Visualization}

The aim of visualization is to produce images to communicate a message. In medical visualization, in particular neurosurgery, it is used to aid in locating regions of interest, such as the tumor, eloquent areas, vessels, among other things, in relation to each other and to the brain. These images can represent data with different dimensions, but here we focus mainly in 3D. It should be noted that the routine to examine the medical data is still in $2 \mathrm{D}$, viewing slice-by-slice. But for image-guided surgical procedures, in particular when tool tracking is performed, $3 \mathrm{D}$ or hybrid methods that combine $2 \mathrm{D}$ and $3 \mathrm{D}$ information are generally used.

As mentioned before, the image modalities produce stacks of slices, i.e. there is one direction in which the scan is performed. To generate images of other views multiplanar reformatting (MPR) is performed. For the head the typical views used are: axial, sagittal and coronal, which represent planes aligned to the three coordinate system axes, i.e. orthogonal planes. If the planes are not orthogonal they are named oblique. In some cases the oblique planes follow a curve or trajectory as used for example in bowel examinations.

In Figure 2.7, there is an example that presents both $2 \mathrm{D}$ and a hybrid 3D method, using the software 3D Slicer [63] ${ }^{2}$. Three 2D images present the axial, sagittal and coronal views and the $3 \mathrm{D}$ view combines two methods, Direct Volume Rendering (DVR) presenting the face and 3D orthogonal planes presenting the orthogonal slices.

DVR was introduced by Tuy et al. [201], Levoy [125] and Drebin et al. [48], it allows the direct generation of an image that represents the 3D scene

\footnotetext{
${ }^{2}$ http://www.slicer.org
} 
(3D image) without performing an initial segmentation to extract a polygonal mesh. To each scalar voxel value a color and opacity (RGBA) value is assigned. This is done with a so-called transfer function (in Figure 2.7 - bottom, left). The most common DVR technique is named ray-casting. In this, from the eye position rays are cast and sampled at fixed intervals. When the opacity level reaches the maximum (opaque) the ray is terminated. The final color is calculated taking into consideration all the samples along the ray. This process is named compositing or alpha-compositing, and it allows the visualization of semi-transparent objects. If only surfaces are desired, the alpha values associated with the scalar values can be either set to full transparency or opacity; this case is named isosurface ray-casting [157, 77] or first-hit ray-casting.

In contrast to direct methods, indirect methods perform a segmentation of the medical volume to obtain polygonal meshes (explicit geometry) of regions of interest, which are subsequently rendered. Alternatively, it is also possible to render the function resulting from the segmentation (contour), known as rendering of implicit surfaces [200, 179].

Furthermore, it is possible to combine direct and indirect volume rendering techniques and generate images containing mixed representations [107, 126].

Most of these techniques along with general concepts of visualization in medicine are well covered in the textbooks $[162,163]$.

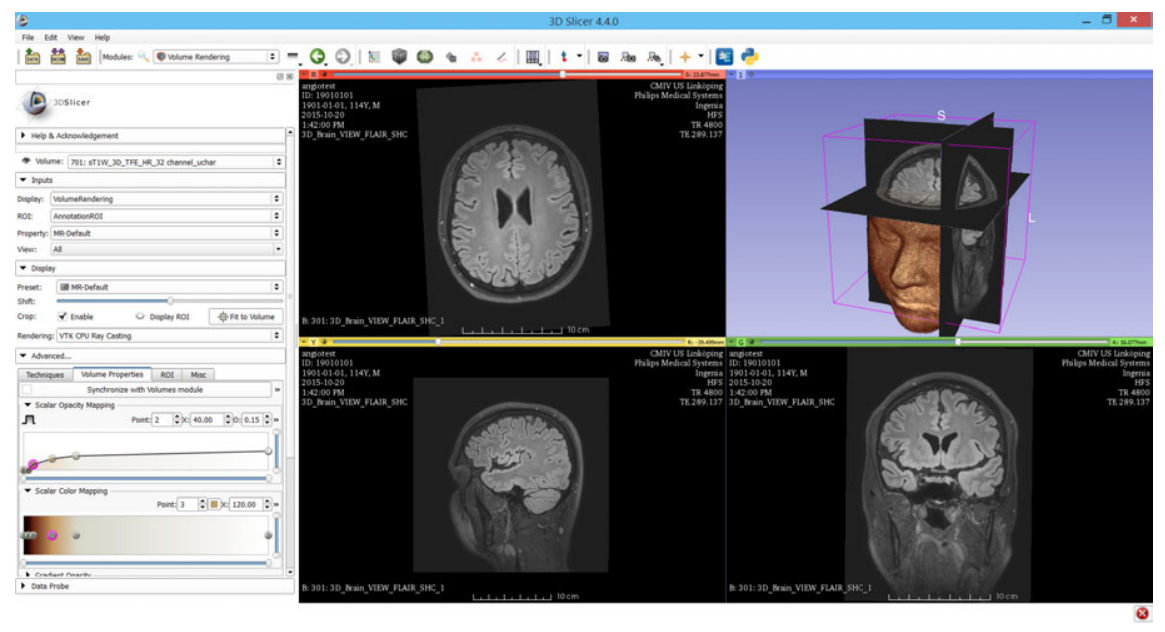

Figure 2.7: Three 2D images present the axial, sagittal and coronal views and the 3D view combines two methods, Direct Volume Rendering (DVR) presenting the face, and 3D orthogonal planes presenting the orthogonal slices. 


\subsection{Image Guided Navigation Systems for Brain Tumor Surgery}

An image-guided surgery uses advanced imaging to localize, target, monitor, and control procedures $[103,101]$. A good survey of intraoperative imaging and image-guided therapy can be found in [64]. Also, an open source development toolkit named: Image-Guided Surgery Toolkit (IGSTK) is available $[56]^{3}$.

There are different system types according to the modality or type of tracking used. In many cases a combination of these types is used. For brain tumor surgery the main types used are:

- Intraoperative MRI (iMRI)

- Intraoperative CT (iCT)

- Intraoperative Ultrasound (iUS)

- Optical

- Electromagnetic

Several commercial systems exist but most do not account for the intraoperative brain shift. Here is presented a review of some works that attempt to deal with this problem. They are organized by their type in the next sections. Tracking of tools is not covered, the focus is on tracking of biological features for brain shift correction. For this reason electromagnetic and optical tool tracking will not be mentioned here.

\subsubsection{Intraoperative MRI}

Using iMRI $[102,142]$ it might not be necessary to correct for brain shift because the dataset is captured intraoperatively and the tumor is visible. But, typically important information e.g. eloquent areas and the tumor, are segmented using the preoperative datasets. This is due to time constrains and typically lower resolution of the intraoperative dataset. If the information contained in the preoperative dataset is required then non-rigid registration is used [47, 215, 32, 6, 84, 62, 132, 207].

Although iMRI allows the visualization of structures of interest directly it has several disadvantages. Cost is for many institutions the main disadvantage since an MRI scanner is a rather expensive machine in comparison to other modalities. Adding to the cost is the fact that a special Operating Room (OR) and tools must be used due to the strong magnetic field. Besides cost, time is also a factor, because it takes considerable time to generate a new MRI dataset. This also makes impractical the acquisition of fMRI data.

\footnotetext{
${ }^{3}$ http: //www .igstk.org/
} 


\subsubsection{Intraoperative CT}

Using iCT many tumors can be seen directly, in particular if contrast agents are administered. It is also possible to use the intraoperative CT data to align it to the preoperative CT or MRI dataset.

This is typically not the preferred method for brain tumor surgery due to the disadvantages of exposure to ionizing radiation, and poor soft tissue delineation, but some groups have explored its use [55, 88]. Haberland et al. [76] also used iCT in combination with iUS.

Although, CT is not used extensively for brain tumor guidance, in other areas of neurosurgery such as vascular surgery Cone Beam Computed Tomography (CBCT), also referred to as C-arm CT, is routinely used [36, 155, 123].

\subsubsection{Intraoperative Ultrasound}

With iUS it is also possible to view directly brain tumors. This is a cost effective and ionizing radiation free modality, which makes it particularly interesting.

The main disadvantage is that compared to MRI it presents many more image artifacts and it requires direct contact to the exposed brain surface. This contact with the brain surface can further increase the brain shift. Furthermore, it might not be possible to capture the full tumor, in particular if the tumor is located on the brain surface.

Several attempts have been made to use the iUS data and align it to preoperative MRI. A good survey of iUS to preoperative MRI registration is provided by Lindseth et al. [130] and a complete system by the same group is presented in [8]. Most of these methods rely on vessel information to perform the registration, but some rely on intensity-based approaches like Mutual Information (MI) [148] or cross-correlation. A summary of the contributions using iUS for brain shift compensation is presented in Table 2.1, organized by: authors of the study, registration method, registration data used to drive the method, perioperative periods and deformation method.

\subsubsection{Optical}

There are several optical techniques applied in tumor surgery, summarized in the next sections. Optical techniques typically do not allow the acquisition of information of the brain interior; instead, the brain surface is captured. Although, with some techniques it is possible to image a few millimeters under the surface, the penetration is limited compared to the previous modalities. An advantage in relation to iUS is that no direct contact with the brain is required. 


\subsection{IMAGE-GUIDED NAVIGATION SYSTEMS FOR BRAIN TUMOR

Table 2.1: Summary of contributions using iUS for brain shift compensation. In front of the authors' information $p$ is used if a phantom object was used in the study, and $i v$ if an in vivo human brain was used.

\begin{tabular}{|c|c|c|c|c|}
\hline Authors & Registration Method & Registration Data & $\begin{array}{l}\text { Perioperative } \\
\text { Periods }\end{array}$ & $\begin{array}{l}\text { Deformation } \\
\text { Method }\end{array}$ \\
\hline $\begin{array}{l}\text { Buchholz et al. } \\
1997[22](i v)\end{array}$ & $\begin{array}{l}\text { 2D spring model placed in a } \\
\text { grid-like fashion + manual se- } \\
\text { lection of homologous points }\end{array}$ & $\begin{array}{l}\text { 2D images points (blood ves- } \\
\text { sels, sulci and ventricles) }\end{array}$ & $\begin{array}{l}\text { preoperative- } \\
\text { intraoperative }\end{array}$ & - \\
\hline $\begin{array}{l}\text { Lunn et al. } \\
2001[134](p, i v)\end{array}$ & SVD & $\begin{array}{l}\text { 3D feature points (blood ves- } \\
\text { sels, sulci and ventricles) }\end{array}$ & $\begin{array}{l}\text { preoperative-- } \\
\text { intraoperative }\end{array}$ & biomechanical \\
\hline $\begin{array}{l}\text { Reinertsen et al. } \\
2007[166](p, i v)\end{array}$ & modified ICP & 3D vessel centerlines & $\begin{array}{l}\text { preoperative- } \\
\text { intraoperative }\end{array}$ & TPS \\
\hline $\begin{array}{l}\text { Reinertsen et al. } \\
2007[168](i v)\end{array}$ & modified ICP & 3D vessel centerlines & $\begin{array}{l}\text { preoperative- } \\
\text { intraoperative }\end{array}$ & TPS \\
\hline $\begin{array}{l}\text { Ji et al. } \\
2008[100] \\
(i v)\end{array}$ & MI & $\begin{array}{l}\text { preprocessed MRI and iUS im- } \\
\text { ages }\end{array}$ & $\begin{array}{l}\text { preoperative- } \\
\text { intraoperative }\end{array}$ & - \\
\hline $\begin{array}{l}\text { Ji et al. } \\
2010[96] \\
(i v)\end{array}$ & B-Spline + MI & $\begin{array}{l}\text { preprocessed MRI and iUS im- } \\
\text { ages }\end{array}$ & $\begin{array}{l}\text { preoperative-- } \\
\text { intraoperative }\end{array}$ & biomechanical \\
\hline $\begin{array}{l}\text { Farnia et al. } \\
2011[60] \\
(i v)\end{array}$ & CPD & $\begin{array}{l}\text { 3D centerlines of plastic tubes } \\
\text { (similar to blood vessels) }\end{array}$ & $\begin{array}{l}\text { preoperative- } \\
\text { intraoperative }\end{array}$ & $\overline{-}$ \\
\hline $\begin{array}{l}\text { Mercier et al. } \\
2012[138](i v)\end{array}$ & $\begin{array}{lll}\begin{array}{l}\text { cross-correlation } \\
\text { ized MI }\end{array} & \text { nor normal- } \\
\end{array}$ & $\begin{array}{l}\text { iUS and pseudo-US (computed } \\
\text { using MRI) images }\end{array}$ & $\begin{array}{l}\text { preoperative- } \\
\text { intraoperative }\end{array}$ & - \\
\hline $\begin{array}{l}\text { Chen et al. } \\
2012[29](p, i v)\end{array}$ & $\begin{array}{l}\text { hybrid feature and intensity- } \\
\text { based (cross-correlation }+ \text { AN- } \\
\text { IMAL [33]) nonlinear registra- } \\
\text { tion }\end{array}$ & 3D blood vessels & $\begin{array}{l}\text { preoperative-- } \\
\text { intraoperative }\end{array}$ & TPS \\
\hline $\begin{array}{l}\text { Reinertsen et al. } \\
2014[167](i v)\end{array}$ & modified ICP & 3D vessel centerlines & $\begin{array}{l}\text { preoperative- } \\
\text { intraoperative }\end{array}$ & - \\
\hline $\begin{array}{l}\text { Farnia et al. } \\
2015[61](p, i v)\end{array}$ & $\begin{array}{l}\text { wavelet based residual com- } \\
\text { plexity }\end{array}$ & $\begin{array}{l}\text { echogenic features such as } \\
\text { sulci, boundary of tumors and } \\
\text { lesions }\end{array}$ & $\begin{array}{l}\text { preoperative- } \\
\text { intraoperative }\end{array}$ & - \\
\hline
\end{tabular}

\section{Laser range scanners}

Roberts et al. [170] presented one of the first solutions using lasers to measure brain surface displacement. A pair of laser beams within the optics of the surgical microscope was used. These beams, converge at the microscope's focal point, allowing the reconstruction of single points.

Solutions where a laser line or point is projected onto an object quickly sweeping it are named Laser Range Scanner (LRS). A camera is used to capture the image of the surface and triangulation is performed to obtain a 3D surface point cloud. LRS systems have been used in surgery to capture the brain surface and to register it to preoperative and intraoperative datasets. A summary of the contributions using LRS systems for brain shift compensation is presented in Table 2.2, organized by: authors of the study, registration method, registration data used to drive the method, perioperative periods and deformation method.

A new generation of LRS was recently presented and evaluated by Pheiffer et al. [159].

\section{Single-view}

Using just one camera, Nakajima et al. [149] registered 2D intraoperative images with $2 \mathrm{D}$ projections of the preoperative MRI dataset. In this case, only a 2D registration using corresponding blood vessels was attempted. Berkels et al. [13] also used a similar registration approach but using sulci 


\subsection{IMAGE-GUIDED NAVIGATION SYSTEMS FOR BRAIN TUMOR SURGERY

Table 2.2: Summary of contributions using LRS systems for brain shift compensation. In front of the authors' information, $p$ is used if a phantom object was used in the study, $i v$ if an in vivo human brain was used and $e v$ if an ex vivo human brain was used.

\begin{tabular}{|c|c|c|c|c|}
\hline Authors & Registration Method & Registration Data & $\begin{array}{l}\text { Perioperative } \\
\text { Periods }\end{array}$ & $\begin{array}{l}\text { Deformation } \\
\text { Method }\end{array}$ \\
\hline $\begin{array}{l}\text { Miga et al. } \\
2001[139] \\
(p, e v)\end{array}$ & $\mathrm{ICP}$ & 3D surface points & $\begin{array}{l}\text { preoperative- } \\
\text { intraoperative }\end{array}$ & biomechanical \\
\hline $\begin{array}{l}\text { Sinha et al. } \\
2002[181] \\
(p, i v)\end{array}$ & surface MI & textured 3D surface points & $\begin{array}{l}\text { preoperative- } \\
\text { intraoperative }\end{array}$ & - \\
\hline $\begin{array}{l}\text { Audette et al. } \\
2003[9] \\
(p, i v)\end{array}$ & surface spline ICP & 3D surface points & $\begin{array}{l}\text { preoperative- } \\
\text { intraoperative }\end{array}$ & biomechanical \\
\hline $\begin{array}{l}\text { Miga et al. } \\
2003[140] \\
(p, i v)\end{array}$ & surface MI & textured 3D surface points & $\begin{array}{l}\text { preoperative- } \\
\text { intraoperative }\end{array}$ & - \\
\hline $\begin{array}{l}\text { Sinha et al. } \\
2003[182] \\
(p, i v)\end{array}$ & $2 \mathrm{D} \mathrm{MI}$ & $2 \mathrm{D}$ images $+3 \mathrm{D}$ surface points & $\begin{array}{l}\text { intraoperative- } \\
\text { intraoperative }\end{array}$ & - \\
\hline $\begin{array}{l}\text { Sinha et al. } \\
2003[183] \\
(p, i v)\end{array}$ & surface MI & textured 3D surface points & $\begin{array}{l}\text { preoperative- } \\
\text { intraoperative }\end{array}$ & - \\
\hline $\begin{array}{l}\text { Duay et al. } \\
2003[50] \\
(p, i v)\end{array}$ & $\begin{array}{l}\text { 2D MI + RBF with finite sup- } \\
\text { port }\end{array}$ & $2 \mathrm{D}$ images & $\begin{array}{l}\text { intraoperative- } \\
\text { intraoperative }\end{array}$ & - \\
\hline $\begin{array}{l}\text { Sinha et al. } \\
2005[180] \\
(p, i v)\end{array}$ & $\begin{array}{l}\text { 2D MI + RBF with finite sup- } \\
\text { port or surface MI }\end{array}$ & $\begin{array}{l}2 \mathrm{D} \text { images }+3 \mathrm{D} \text { surface points } \\
\text { or textured } 3 \mathrm{D} \text { surface points }\end{array}$ & $\begin{array}{l}\text { intraoperative- } \\
\text { intraoperative }\end{array}$ & - \\
\hline $\begin{array}{l}\text { Cao et al. } \\
2007 \text { [23] } \\
\text { (iv) }\end{array}$ & $\begin{array}{l}\text { feature PBR or vessel ICP or } \\
\text { SurfaceMI }\end{array}$ & $\begin{array}{l}3 \mathrm{D} \text { feature points or } 3 \mathrm{D} \text { blood } \\
\text { vessel centerlines or textured } \\
3 \mathrm{D} \text { surface points }\end{array}$ & $\begin{array}{l}\text { preoperative- } \\
\text { intraoperative }\end{array}$ & - \\
\hline $\begin{array}{l}\text { Ding et al. } \\
2007 \text { [45] } \\
(i v)\end{array}$ & 2D TPS-RPM or 3D TPS-RPM & $\begin{array}{l}2 \mathrm{D} \text { blood vessel centerlines }+ \\
3 \mathrm{D} \text { surface points or } 3 \mathrm{D} \text { blood } \\
\text { vessel centerlines }\end{array}$ & $\begin{array}{l}\text { intraoperative- } \\
\text { intraoperative }\end{array}$ & TPS \\
\hline $\begin{array}{l}\text { Cao et al. } \\
2008[24] \\
(i v)\end{array}$ & $\begin{array}{l}\text { feature PBR or vessel ICP or } \\
\text { SurfaceMI or constrained Sur- } \\
\text { faceMI }\end{array}$ & $\begin{array}{l}\text { 3D feature points or 3D blood } \\
\text { vessel centerlines or textured } \\
\text { 3D surface points }\end{array}$ & $\begin{array}{l}\text { preoperative- } \\
\text { intraoperative }\end{array}$ & - \\
\hline $\begin{array}{l}\text { Ding et al. } \\
2009[46] \\
(i v)\end{array}$ & - & 2D blood vessel centerlines & $\begin{array}{l}\text { intraoperative- } \\
\text { intraoperative }\end{array}$ & - \\
\hline $\begin{array}{l}\text { Chen et al. } \\
2011[28] \\
(i v)\end{array}$ & $\begin{array}{l}\text { atlas based + constrained Sur- } \\
\text { faceMI }\end{array}$ & textured 3D surface points & $\begin{array}{l}\text { preooperative- } \\
\text { intraoperative }\end{array}$ & biomechanical \\
\hline $\begin{array}{l}\text { Sun et al. } \\
2014[195] \\
\text { (iv) }\end{array}$ & $\begin{array}{l}\text { atlas based }+ \text { manual selection } \\
\text { of homologous points }\end{array}$ & $\begin{array}{l}\text { 3D blood vessel bifurcation } \\
\text { points }\end{array}$ & $\begin{array}{l}\text { preooperative- } \\
\text { intraoperative }\end{array}$ & biomechanical \\
\hline $\begin{array}{l}\text { Miga et al. } \\
2015[141] \\
(i v)\end{array}$ & $\begin{array}{l}\text { atlas based }+ \text { manual selection } \\
\text { of homologous points }\end{array}$ & $\begin{array}{l}\text { 3D blood vessel bifurcation } \\
\text { points }\end{array}$ & $\begin{array}{l}\text { preooperative- } \\
\text { intraoperative }\end{array}$ & biomechanical \\
\hline
\end{tabular}

information instead of vessels.

Instead of using preoperative MRI projections, Ding et al. [44, 42, 43] proposed to register continuous video sequences acquired by a camera integrated in the surgical microscope to either images previously acquired by the microscope or images acquired by the LRS at some previous time points (the interval can be large). Vessels were also used as registration features.

Recently, Jiang et al. [95] used a single camera system in combination with a small projector that was used to project a phase-shift image on the brain surface (tested with a porcine phantom). The image was used to aid in the surface reconstruction process. Furthermore, for brain shift compensation the registration used reconstructed surface points and vessel/sulci feature points. 


\section{Two-view}

Similar to the LRS, 3D points on the brain surface can also be obtained using two images. These can be acquired with camera systems that in some cases are integrated in the surgical microscope (Figure 2.8). The cameras can either be rigidly attached to the microscope, capturing images different from the microscope optics, or use beam splitters capturing images as seen by the microscope optics. One important distinction between these systems and the LRS is the acquisition time. Typically camera systems can capture multiple images in one second but a LRS takes at least several seconds to acquire the data due to the scanning procedure. This can cause errors because the brain is not an immobile object; instead, it pulsates, mainly due to breathing and heart rate [59]. The average human has one heart beat per second thus the brain surface will change during the LRS acquisition.

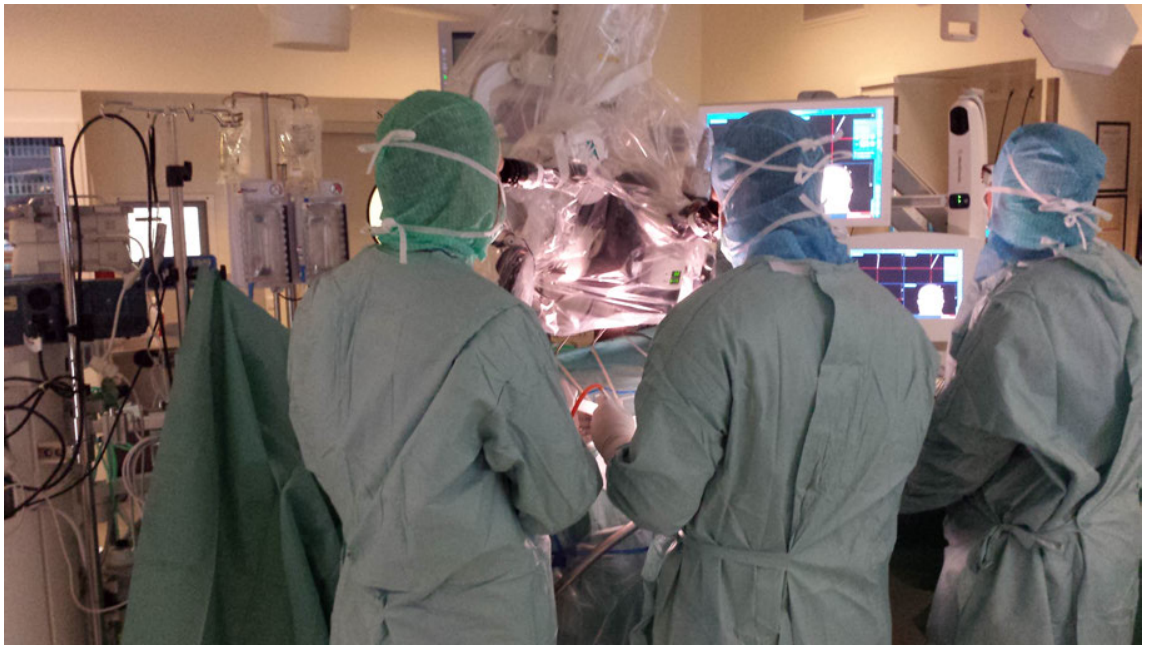

Figure 2.8: Surgical Microscope wrapped in a sterile cover. (Image Courtesy of Per M. Karlsson, MD, Department of Neurosurgery, Linköping University, Linköping, Sweden)

Computer vision techniques are used to reconstruct points on the brain surface. Many techniques exist but they are mainly of two types:

- feature-based

- intensity-based

A feature-based approach first extracts features in the pair of images and then finds the correspondences between the homologous feature positions, known as the correspondence problem. Once this procedure is complete, triangulation is performed to obtain the $3 \mathrm{D}$ point/feature positions. Typical 


\subsection{IMAGE-GUIDED NAVIGATION SYSTEMS FOR BRAIN TUMOR SURGERY

features used for brain shift compensation using two-view systems are: sulci, vessels and vessel bifurcations.

To identify the homologous points, the intensity-based approach uses image intensity values and similarity measures are performed to identify the best candidates. DeLorenzo et al. [40,41] combined these two approaches using the sulci as features.

The registration typically uses the intraoperative surface points and/or

Table 2.3: Summary of contributions using two-view systems for brain shift compensation. In front of the authors' information, $p$ is used if a phantom object was used in the study, $i v$ if an in vivo human brain was used, ivA if an in vivo animal brain was used, $v$ if a virtual human brain was used and SM if the cameras were integrated in a surgical microscope.

\begin{tabular}{|c|c|c|c|c|}
\hline Authors & Registration Method & Registration Data & $\begin{array}{l}\text { Perioperative } \\
\text { Periods }\end{array}$ & $\begin{array}{l}\text { Deformation } \\
\text { Method }\end{array}$ \\
\hline $\begin{array}{l}\text { Skrinjar et al. } \\
2001[211] \\
(i v, v)\end{array}$ & 2D normalized MI & $2 \mathrm{D}$ images & $\begin{array}{l}\text { preoperative- } \\
\text { intraoperative }\end{array}$ & biomechanical \\
\hline $\begin{array}{l}\text { Skrinjar et al. } \\
2002[210] \\
(i v, v)\end{array}$ & $\begin{array}{l}\text { 2D normalized } \\
\text { correlation }\end{array}$ & $2 \mathrm{D}$ images & $\begin{array}{l}\text { preoperative- } \\
\text { intraoperative }\end{array}$ & biomechanical \\
\hline $\begin{array}{l}\text { Sun et al. } \\
2003[193] \\
(p, i v, \mathrm{SM})\end{array}$ & $\mathrm{ICP}$ & $\begin{array}{l}3 \mathrm{D} \text { blood vessel centerlines } \\
\text { (initial) }+3 \mathrm{D} \text { surface points }\end{array}$ & $\begin{array}{l}\text { preoperative- } \\
\text { intraoperative }\end{array}$ & biomechanical \\
\hline $\begin{array}{l}\text { Sun et al. } \\
2003[192] \\
(i v, \text { SM })\end{array}$ & ICP or gravitational sagging & 3D surface points & $\begin{array}{l}\text { preoperative- } \\
\text { intraoperative }\end{array}$ & biomechanical \\
\hline $\begin{array}{l}\text { Sun et al. } \\
2005[194] \\
(p, i v, \mathrm{SM}) \\
\end{array}$ & ICP & 3D surface points & $\begin{array}{l}\text { preoperative- } \\
\text { intraoperative }\end{array}$ & biomechanical \\
\hline $\begin{array}{l}\text { DeLorenzo et al. } \\
2006[40] \\
(p, i v)\end{array}$ & Bayesian analysis & $\begin{array}{l}2 \mathrm{D} \text { images }+2 \mathrm{D} \text { sulci }+3 \mathrm{D} \\
\text { sulci }\end{array}$ & $\begin{array}{l}\text { preoperative- } \\
\text { intraoperative }\end{array}$ & biomechanical \\
\hline $\begin{array}{l}\text { DeLorenzo et al. } \\
2006[41] \\
(i v)\end{array}$ & Bayesian analysis & $\begin{array}{l}2 \mathrm{D} \text { images }+2 \mathrm{D} \text { sulci }+3 \mathrm{D} \\
\text { sulci }\end{array}$ & $\begin{array}{l}\text { preoperative- } \\
\text { intraoperative }\end{array}$ & biomechanical \\
\hline $\begin{array}{l}\text { Paul et al. } \\
2009[158] \\
(p, i v, \mathrm{SM})\end{array}$ & $\begin{array}{l}\text { hidden Markov process }(2 \mathrm{D} \\
\text { point registration); } \\
\text { (initialization) and intensity- } \\
\text { based optimization (surface } \\
\text { registration) }\end{array}$ & $\begin{array}{l}\text { 2D points }+3 \mathrm{D} \text { surface points } \\
+2 \mathrm{D} \text { images }\end{array}$ & $\begin{array}{l}\text { intraoperative- } \\
\text { intraoperative }\end{array}$ & - \\
\hline $\begin{array}{l}\text { DeLorenzo et al. } \\
2010[39] \\
(i v)\end{array}$ & game theory & $\begin{array}{l}2 \mathrm{D} \text { images }+2 \mathrm{D} \text { sulci }+3 \mathrm{D} \\
\text { sulci }\end{array}$ & $\begin{array}{l}\text { preoperative- } \\
\text { intraoperative }\end{array}$ & - \\
\hline $\begin{array}{l}\text { Ji et al. } \\
2010[96] \\
(i v, \text { SM }) \\
\end{array}$ & rigid & fiducial markers & $\begin{array}{l}\text { preoperative- } \\
\text { intraoperative }\end{array}$ & biomechanical \\
\hline $\begin{array}{l}\text { Ji et al. } \\
2011[98] \\
(i v, \text { SM })\end{array}$ & optical flow & $2 \mathrm{D}$ images & $\begin{array}{l}\text { intraoperative- } \\
\text { intraoperative }\end{array}$ & - \\
\hline $\begin{array}{l}\text { Vivanti et al. } \\
2013[208] \\
(p)\end{array}$ & video tracking & $2 \mathrm{D}$ images $+2 \mathrm{D}$ points & $\begin{array}{l}\text { intraoperative- } \\
\text { intraoperative }\end{array}$ & - \\
\hline $\begin{array}{l}\text { Ahmadian et al. } \\
2013[2] \\
(p, i v A)\end{array}$ & modified ICP & 3D surface points & $\begin{array}{l}\text { intraoperative- } \\
\text { intraoperative }\end{array}$ & - \\
\hline $\begin{array}{l}\text { Faria et al. } \\
2014[59] \\
(p, i v)\end{array}$ & video tracking & $2 \mathrm{D}$ images $+2 \mathrm{D}$ points & $\begin{array}{l}\text { intraoperative- } \\
\text { intraoperative }\end{array}$ & - \\
\hline $\begin{array}{l}\text { Ji et al. } \\
2014[99] \\
(p, i v, \mathrm{SM}) \\
\end{array}$ & optical flow & $2 \mathrm{D}$ images & $\begin{array}{l}\text { intraoperative- } \\
\text { intraoperative }\end{array}$ & - \\
\hline $\begin{array}{l}\text { Ji et al. } \\
2014[97] \\
(p, i v, \mathrm{SM})\end{array}$ & optical flow & $2 \mathrm{D}$ images & $\begin{array}{l}\text { intraoperative- } \\
\text { intraoperative }\end{array}$ & $\overline{-}$ \\
\hline $\begin{array}{l}\text { Fan et al. } \\
2014[57] \\
(i v, \mathrm{SM})\end{array}$ & $\begin{array}{l}\text { sum-of-squared-differences } \\
\text { rigid }+2 \mathrm{D} \text { MI }\end{array}$ & $2 \mathrm{D}$ images & $\begin{array}{l}\text { preoperative- } \\
\text { intraoperative }\end{array}$ & biomechanical \\
\hline $\begin{array}{l}\text { Kumar et al. } \\
2014[114] \\
(i v, \mathrm{SM})\end{array}$ & rigid + TPS & $3 \mathrm{D}$ points & $\begin{array}{l}\text { intraoperative- } \\
\text { intraoperative }\end{array}$ & - \\
\hline
\end{tabular}


features points from vessels or sulci to align them to the correspondent preoperative MRI data or intraoperative data. A summary of the contributions using two-view systems for brain shift compensation is presented in Table 2.3, organized by: authors of the study, registration method, registration data used to drive the method, perioperative periods and deformation method.

A comparison between the accuracy of the LRS and the two-view (stereo) system was presented by Kumar et al. [116]. Hybrid systems combining two-view systems and iUS have also been proposed by Ji et al. [96] and Mohammadi et al. [143].

Solutions where a light pattern is projected onto the brain to aid the surface reconstruction were studied by Ahmadian et al. [2] and Mohammadi et al. [143]. This type of technique is known as structured light.

Recently, Yang et al. [226] developed a craniotomy simulation system for evaluation of stereo-pair reconstruction fidelity and tracking.

Regarding solutions where cameras are integrated in the surgical microscope, Kumar et al. [115] recently explored the possibility of persistently and automatically reconstructing intraoperative surfaces using dynamic magnifications of an operating microscope.

\section{Three-view}

Three-view systems are not as common as two-view systems. A three-view system was develop in this work, and an image of our system is presented in Figure 2.9. Besides our work, D'Apuzzo and Verius [37] also used three cameras to reconstruct points on the brain surface.

\section{Fluorescence}

With fluorescence techniques it is also possible to view directly some tumor types. Typically a drug (photosensitizer) is administered that makes the tissue of interest emit light at a certain frequency.

There are two main types of systems:

- fluorescence microscopy

- fiber-optic based fluorescence spectroscopy

In our context, fluorescence microscopy refers to systems where images of the brain are acquired with a surgical microscope. In these images it is possible to view the tissue of interest emitting light of a specific color by administering a photosensitizer. The most common photosensitizer used for tumor visualization is the 5-Aminolevulinic Acid (5-ALA) [188, 189, 161, 190, 191, 219]. There are commercially available solutions from several vendors such as: Carl Zeiss (Oberkochen, Germany) ${ }^{4}$ and Leica

\footnotetext{
${ }^{4}$ http://www.zeiss.com/meditec/en_de/products---solutions/neurosurgery/ intraoperative-fluorescence/tumor/blue-400.html
} 


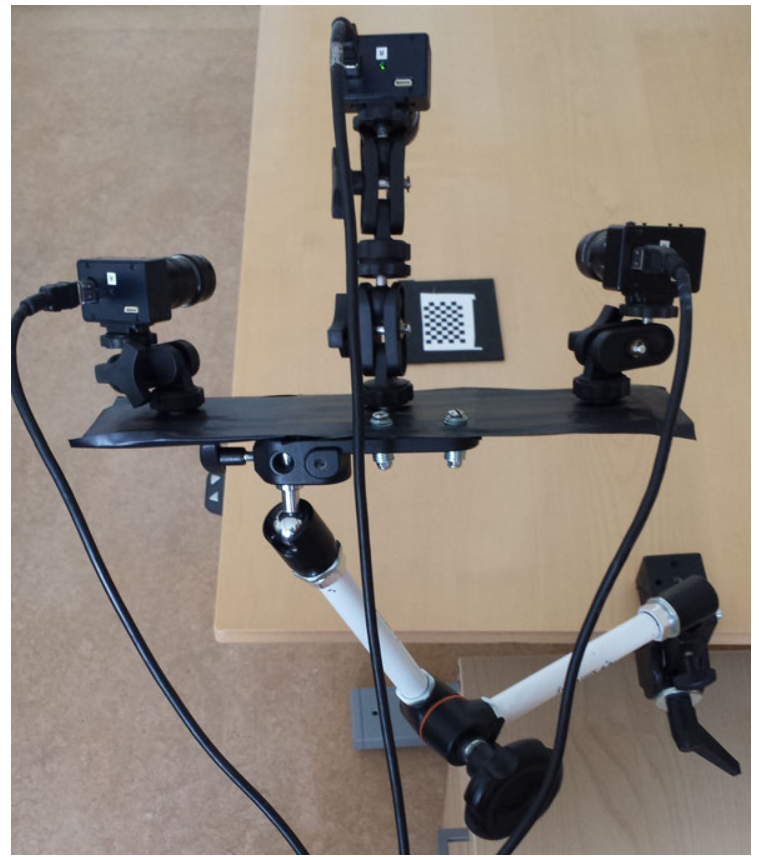

Figure 2.9: Our three-view camera system.

Microsystems (Wetzlar, Germany) ${ }^{5}$ surgical microscopes. In Figure 2.10 it is possible to see the brain surface under white light and under blue (ultraviolet) light with ALA. Under blue light the tumor appears pink or red. This technique has been beneficial to identify highly malignant gliomas; however, the low grade gliomas and a percentage of some other tumor types do not take up ALA sufficiently to be visualized with a microscope.

Similar to ALA it is also possible to use sodium fluorescein [127]. In this case the tumor will be visible in yellow (Figure 2.11) due to the filter used. A commercial solution is provided by Carl Zeiss ${ }^{6}$.

Another popular fluorescence microscopy technique is Indocyanine Green (ICG) $[119,164,165,38,196,5,203]$ used mainly to image blood vessels, which are seen as bright white (Figure 2.12). This technique requires nearinfrared (NIR) imaging and is integrated in commercial surgical microscopes from several vendors such as Carl Zeiss ${ }^{7}$ and Leica ${ }^{8}$.

\footnotetext{
${ }^{5}$ http://www.leica-microsystems.com/science-lab/surgical-microscopy/ if-it-glows-its-tumor-5-ala-fluorecence-visualization/

${ }^{6}$ http://www.zeiss.com/meditec/en_de/products---solutions/neurosurgery/ intraoperative-fluorescence/tumor/yellow-560.html

${ }^{7}$ http://www.zeiss.com/meditec/en_de/products---solutions/neurosurgery/ intraoperative-fluorescence/vascular/infrared-800.html

${ }^{8}$ http://www.leica-microsystems.com/products/surgical-microscopes/ neurosurgery-spine/details/product/leica-fl800/
} 

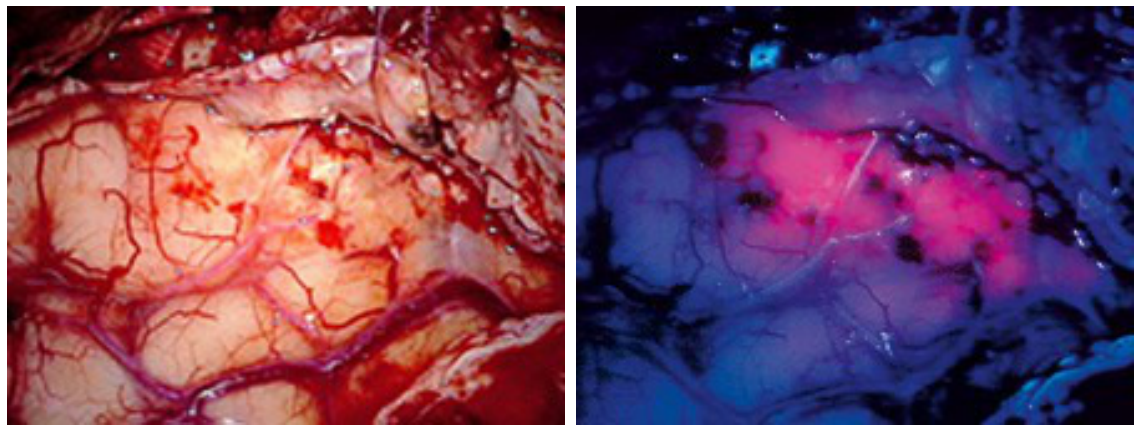

Figure 2.10: Brain surface under white light (left) and under blue light with ALA (right). Under blue light the tumor is visible in pink. (Image Courtesy of Walter Stummer, MD, PhD, Department of Neurosurgery, University Hospital Muenster, Muenster, Germany).

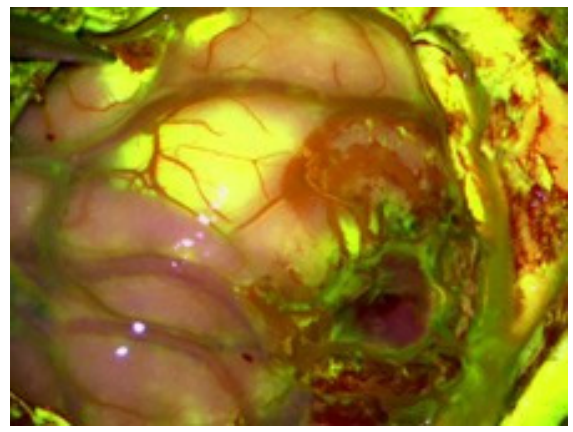

Figure 2.11: Brain surface with sodium fluorescein, the tumor is visible in yellow. (Image Courtesy of Aaron A. Cohen-Gadol, MD, MSc, Department of Neurosurgery, Goodman Campbell Brain and Spine, Indianapolis, USA).

Recently an augmented reality system using ICG was proposed by Watson et al. [216], where vessels visible with ICG are segmented and merged with white light microscope images.

Fiber-optic based fluorescence spectroscopy, as the name indicates uses a handheld fiber-optic probe to transmit the light to and from a small area of the brain. For brain tumor demarcation Li et al. [129, 128] created one of the first systems. Lately, fiber-optic systems for detection of ALA have been developed [202, 92, 223, 81, 205, 169, 79, 78, 82], including attempts to quantify and correct for photo-bleaching problems and tissue attenuation $[80,21,204]$. 


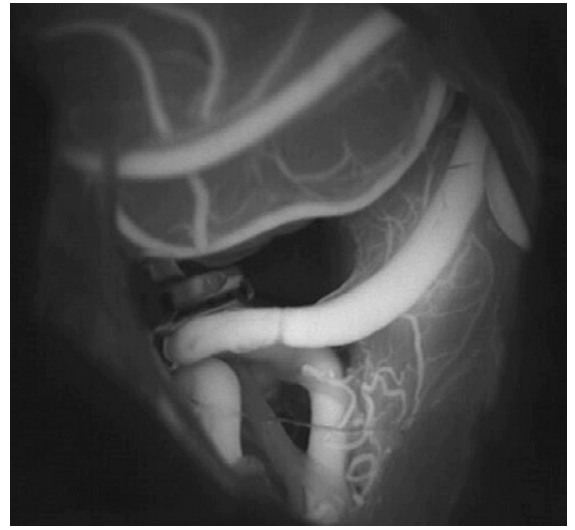

Figure 2.12: Brain surface with ICG, the vessels are visible as bright white. (Image Courtesy Prof. Dr. Andreas Raabe, Department of Neurosurgery, Bern University Hospital, Bern, Switzerland).

\subsection{Stereoscopy and Depth Perception of Semi- Transparent Objects}

Stereoscopy is a technique for creating or enhancing the illusion of depth by means of stereopsis or binocular vision. It requires the generation of a pair of images (stereoscopic images), and each image is viewed individually by each eye. To produce and display these images there exist a number of stereoscopic display systems. The differences between stereoscopic technologies lie in how the images are presented to the viewer.

In this work, only systems with relatively large screens, such as typical monitors, are considered. Head-mounted displays and similar systems that make use of small displays are excluded.

The most common active stereo display technique is shutter glasses, where the display is synchronized with the opening and closing of the shutters of the glasses, so that the viewer observes just one image at a time $[153]^{9},[10]^{10}$. The drawback is the synchronization process, which might not work perfectly, producing ghost artifacts (crosstalk) [220], and there is a potential risk of triggering epileptic seizures in the case of prolonged usage due to flickering. Active shutter glasses may also produce ghosting due to their liquid cristal shutters response times, imperfect masking, etc. Other passive systems include:

- anaglyphs, that use glasses with color filters to filter the images, limiting the usage of color. Ghosting can occur as the color filters are not perfect;

\footnotetext{
${ }^{9}$ http://www.nvidia.com/object/3d-vision-main.html

${ }^{10}$ http://www.stereo3d.com/Barco_Stereoscopic_proj.pdf
} 
- polarized, where special screens are used to display images with different light polarizations and special glasses are required. They suffer from ghosting due to light polarization crosstalk;

- wavelength multiplex imaging [104], where light is separated into three spectral ranges and special glasses are used with filters for specific spectral ranges. It also suffers from ghosting due to spectral range overlapping.

There are also autostereoscopic displays from several brands in the market. Two examples of this are parallax barrier and lenticular autostereoscopic displays $[14,94]$. In fact, the principles of this technology are rather old, but were made available by the new LCD screen technology. More complete surveys of stereo display systems can be found in $[86,113,86,72]$.

Dual-monitor systems $[113,65,131]$ can also be used for stereoscopic viewing. The Planar StereoMirror 3D Monitor ${ }^{11}$ is an example that uses a semi-transparent polarized mirror.

Most similar to the system developed in this work is the dual-monitor single-mirror system originally proposed by Hart ${ }^{12}$, [221] and the stereoscopic system with a single-monitor ${ }^{13}$ also used by Hart.

Stereopsis or binocular disparity is one of the most important binocular depth cues for close range visualization (personal space or action space) [35]. In this work a new stereoscopic display was used to study the depth perception of semi-transparent virtual objects and their spatial layout. The correct visualization of the object's spatial position is crucial in many applications. Medical applications are a good example of where accurate visualization is required, in particular if surgical procedures are dependent on the visualization. Sielhorst et al. [178] and Bianchi [16] present several surgical augmented reality visualization systems. The da Vinci (Intuitive Surgical Inc., Mountain View, CA, USA) ${ }^{14}$ system is another example of a surgical stereoscopic system, also with augmented reality possibilities [209]. Recently, an augmented reality training system for planning tumor resection was presented by Abhari et al. [1].

Extensive literature regarding depth perception and shape perception exists. For depth perception the various depth cues, their strengths and relationships are explored by Cutting and Vishton [35]. The depth cues identified by Cutting and Vishton are:

- Occlusion

- Height in the visual field

- Motion perspective

\footnotetext{
${ }^{11}$ http://www.planar.com/support/products/3d-stereoscopic/

${ }^{12}$ http://www . hart3d.com/pages/3d-viewing/stereoformat.html

${ }^{13} \mathrm{http}: / /$ stereo.jpn.org/eng/stphmkr/mirror/mirrorview.htm

${ }^{14} \mathrm{http}: / /$ www. davincisurgery.com/
} 
- Binocular disparities

- Relative size

- Relative density

- Convergence and accommodation

- Aerial perspective

In general, these cues are used with opaque objects, in particular occlusion, which is the strongest depth cue. Regarding shape perception, we can also find specific cues [197] and some of them, such as, motion are common in both cases. There is a clear interdependency between depth and shape.

Perception of transparent 3D objects has been addressed in some studies. Boucheny et al. [19] present a perceptive evaluation of volume rendering techniques. They used DVR techniques, which are commonly used for the visualization of semi-transparent medical data where different tissue types are blended together. Two semi-transparent cylinders were organized in depth, with different widths and luminance values, and a small intersection region on the rendered image. The participants had poor results for the relative size and luminosity cues. Interrante et al. [93] addressed the transparency of enclosed objects to convey 3D shape via texture. Nakayama et al. [150] analyze transparency in relation to depth, subjective contours, luminance, and neon color spreading. Kersten et al. [108] explore the enhancement of depth perception in translucent volumes. A perception-based transparency optimization for DVR is presented by Chan et al. [26] and perceptually based depth-ordering enhancement for DVR by Zheng et al. [228].

Perception of transparency using stereoscopic systems can be found in [3] where the transparency of overlapping surfaces using random-dot stereograms is investigated and in [199], which investigates the constraints on the perception of multiple surfaces also using dot stereograms. Maurer et al. [137] used stereoscopic augmented reality systems to visualize brain structures inside a head phantom with different rendering methods. Since the user can move around the phantom, kinetic cues are also present. Sielhorst et al. [178] developed another augmented reality system where surgeons are asked to locate points on the vertebral column using a pointing tool inside a torso phantom. Multiple rendering methods were also used in this study. In both previous studies it was reported that the results were considerably different for the various rendering methods. 


\section{Chapter 3}

\section{Aims}

The main goal of this work was to create methods and tools to aid surgeons in the safe resection of brain tumors. In particular, the focus is on brain shift compensation, including vessel reconstruction, registration and volume deformation. Aspects of visualization, in particular how the position of a tumor is perceived when enclosed in the brain, were also studied. More specifically, the aims of the dissertation were:

- To develop a method for 3D superficial vessel reconstruction using a multi-view camera system, and evaluate its accuracy;

- To study and develop registration methods (rigid and non-rigid) between preoperative and intraoperative data;

- To implement and evaluate methods for fast volume deformation to compensate for brain shift, using the TPS (direct and indirect approaches) and the correspondences found by registration methods;

- To study how the position of a tumor is perceived in relation to the brain, when the tumor is enclosed in the brain. Using a stereoscopic display and different rendering methods, object positions and color blending (opacity) values. 


\section{Chapter 4}

\section{Summary of the Papers}

\subsection{D Vessel Centerline Reconstruction}

\section{Paper I}

\section{Methods}

A method for reconstruction of 3D brain superficial vessel centerlines using a multi-view (three-view) camera system was developed and evaluated. Vessel centerlines were manually selected in the three images (Figure 4.1 presents one of the three images of a phantom brain). Using the properties of the Hessian matrix, the centerline points are assigned direction information. For correspondence matching, a combination of methods was used. The process starts with epipolar and spatial coherence constraints (geometrical constraints), followed by relaxation labeling and an iterative filtering where the $3 \mathrm{D}$ points are compared to surfaces obtained using the TPS with a decreasing relaxation parameter. Finally, the points are shifted to their local centroid position (mean shift).

The vessel reconstruction evaluation was performed for the various steps of the vessel reconstruction pipeline as presented in Figure 4.2 with three different depth ranges ( $\mathrm{Z}$ coordinate). The depth ranges were used to evaluate the influence of depth on system error. Three different cases were studied, using phantom and virtual datasets:

- RL - used relaxation labeling without TPS filtering and mean shift

- RL+TPS - used relaxation labeling and TPS filtering without mean shift

- RL+TPS+MS - used relaxation labeling, TPS filtering and mean shift

The comparison metrics used the distance from the centerline points of our method to the closest MRI/CT centerline point obtained by Wang's et al. [212] method (aligned by a rigid transformation, if applicable). 


\subsection{D VESSEL CENTERLINE RECONSTRUCTION}

A patient dataset was also used for qualitative visual inspection.

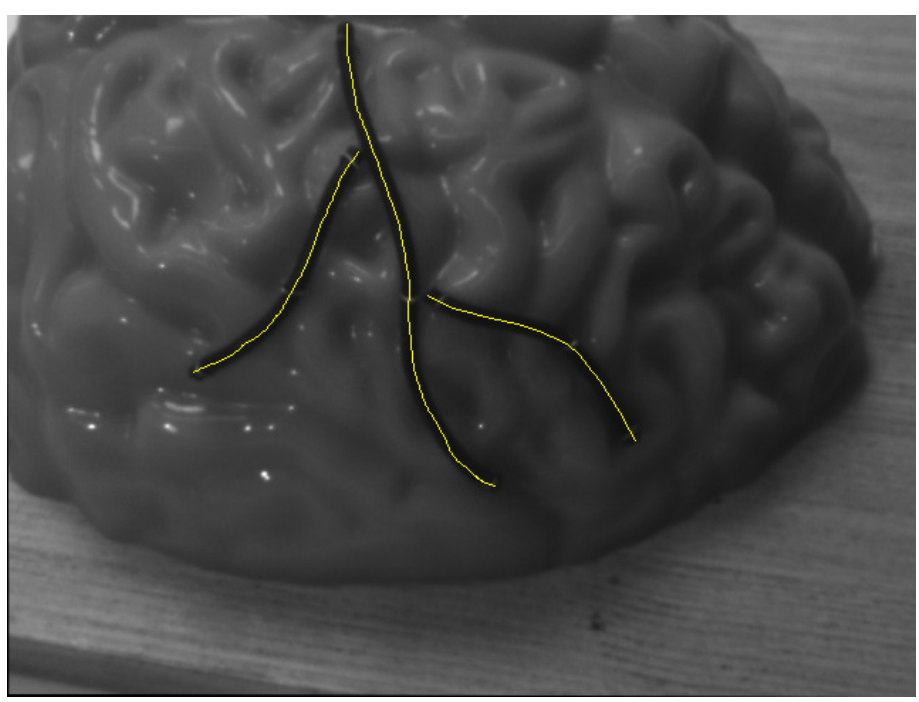

Figure 4.1: One view of the phantom brain. The vessel centerlines manually selected for evaluation are highlighted (yellow).

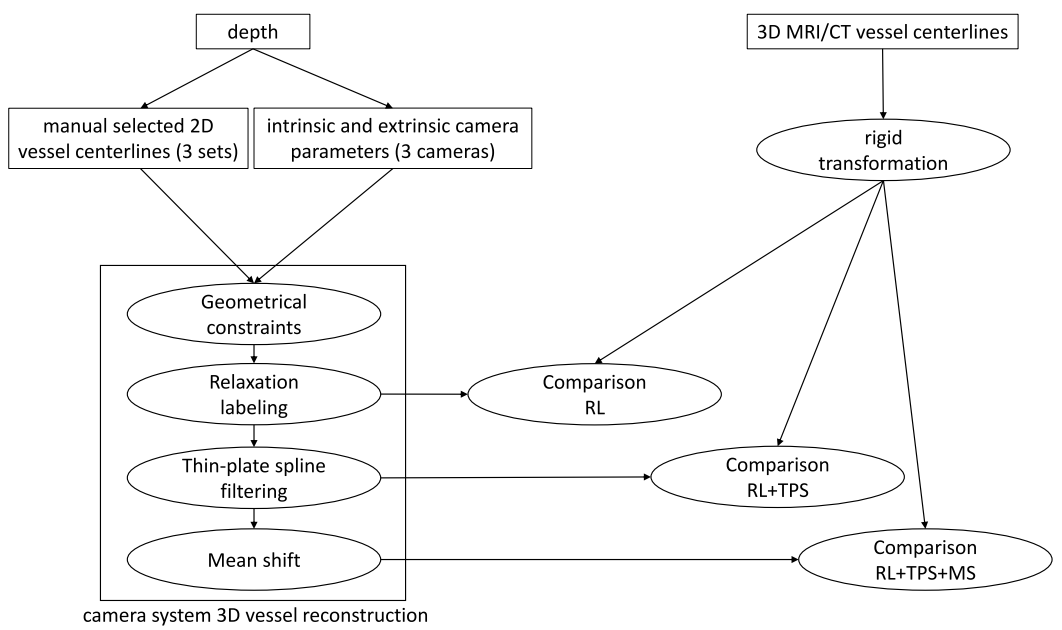

Figure 4.2: Diagram of the vessel centerline reconstruction evaluation. 


\section{Results}

In both experiments (phantom, and virtual datasets) and all depths considered, the accuracy increased when using more pipeline blocks. When using all the pipeline blocks (RL+TPS+ MS), the results were similar for the depths considered, and in all cases, both RMSE and ME were approximately $1 \mathrm{~mm}$. In Figure 4.3, the results for one of the distances tested, using the phantom dataset, are presented.

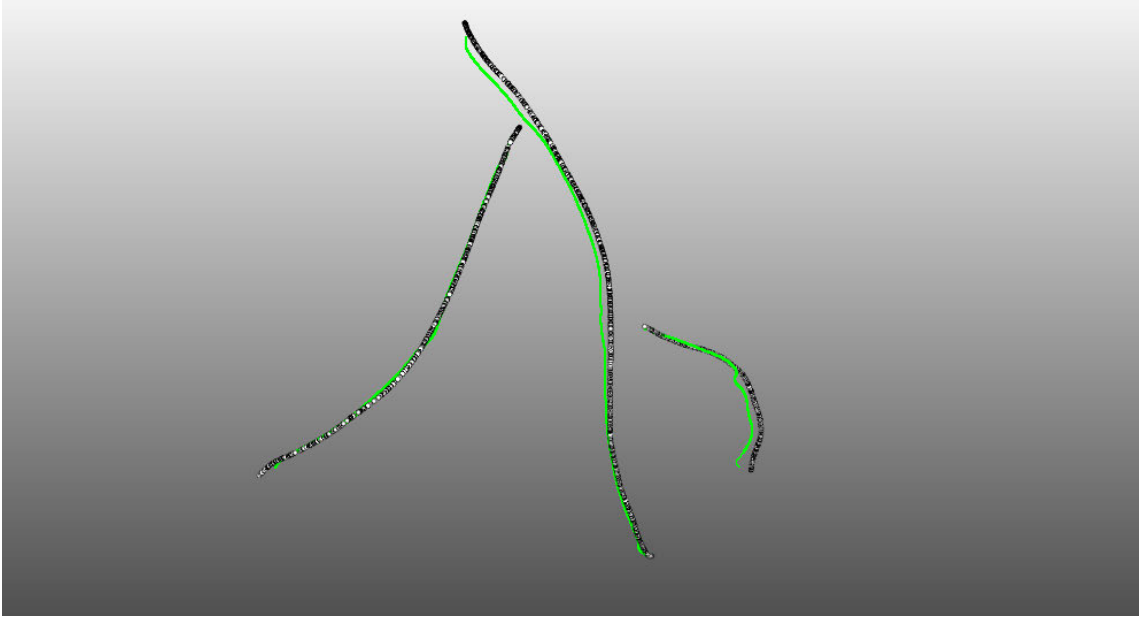

Figure 4.3: 3D phantom centerlines. The CT centerlines are presented in green, and the centerline points obtained using the cameras are presented as white spheres.

A visual evaluation was also performed for a patient dataset; it was possible to reconstruct the complete selected centerlines, and the shape seemed to correspond to the shape of the patient's vessels.

\subsection{Brain Shift Compensation}

\section{Paper II}

\section{Methods}

This work was the first attempt to develop our own complete system for brain shift compensation. The non-rigid registration method uses preoperative and intraoperative superficial blood vessel centerlines of the brain. The preoperative vessel centerlines are segmented from a preoperative MRI [212] and the intraoperative vessel centerlines obtained with a predecessor method to the reconstruction method presented in paper I [136]. Before the non- 
rigid registration a manual rigid registration was performed to transform the points from the cameras coordinate system to the MRI coordinate system. Followed by a non-rigid point set registration method. The non-rigid registration method used was the CPD, which provided the correspondences between the two vessel centerline point sets. Finally, the generation of a new deformed volume was achieved with the TPS method using as control points the correspondences in the MRI coordinate system found in the previous step and extra homologous points with the same position in regions where no deformation was expected in order to constrain the transformation.

The method was tested in a rabbit brain exposed via craniotomy, where deformations were produced by a balloon inserted into the brain.

\section{Results}

A good correlation was found between the real state of the brain and the deformed volume obtained using the pipeline. Maximum displacements were approximately $4.0 \mathrm{~mm}$ for the exposed brain alone, and $6.7 \mathrm{~mm}$ after balloon inflation.

\section{Paper III}

\section{Methods}

Although the results of our previous paper (paper II) were satisfactory, we have found that only using the CPD is not sufficient, mainly because of incomplete vessel regions from the intraoperative datasets. To overcome this problem, a method was developed that consists of a combination of the CPD and the TPS semilandmarks. The CPD is used to perform the initial matching of centerline $3 \mathrm{D}$ points, while the semilandmarks method iteratively relaxes/slides the points.

For the evaluation, a MRA dataset was used. Deformations were applied to the extracted vessel centerlines to simulate brain bulging and sinking, using a TPS deformation where a few control points were manipulated to obtain the desired transformation $\left(T^{1}\right)$. Once the correspondences were known, the corresponding points were used to define a new TPS deformation $\left(T^{2}\right)$. The errors were measured in the deformed space, by transforming the original points using $T^{1}$ and $T^{2}$ and measuring the distance between them. To simulate cases where the deformed vessel data is incomplete, parts of the reference vessels were cut and then deformed. Furthermore, anisotropic normally distributed noise was added.

\section{Results}

Visually the results of our method for the bulging incomplete data case with added noise can be seen in Figure 4.4. For the deformations without noise, similar results were obtained for all cases (sink-complete, bulge-complete, 
sink-incomplete, and bulge-incomplete,). When noise was added, the accuracy was worse in all cases. There seemed to be no clear preference between complete and incomplete data. Even with the presence of noise and incomplete data, the RMSE and ME were well below $1 \mathrm{~mm}$.

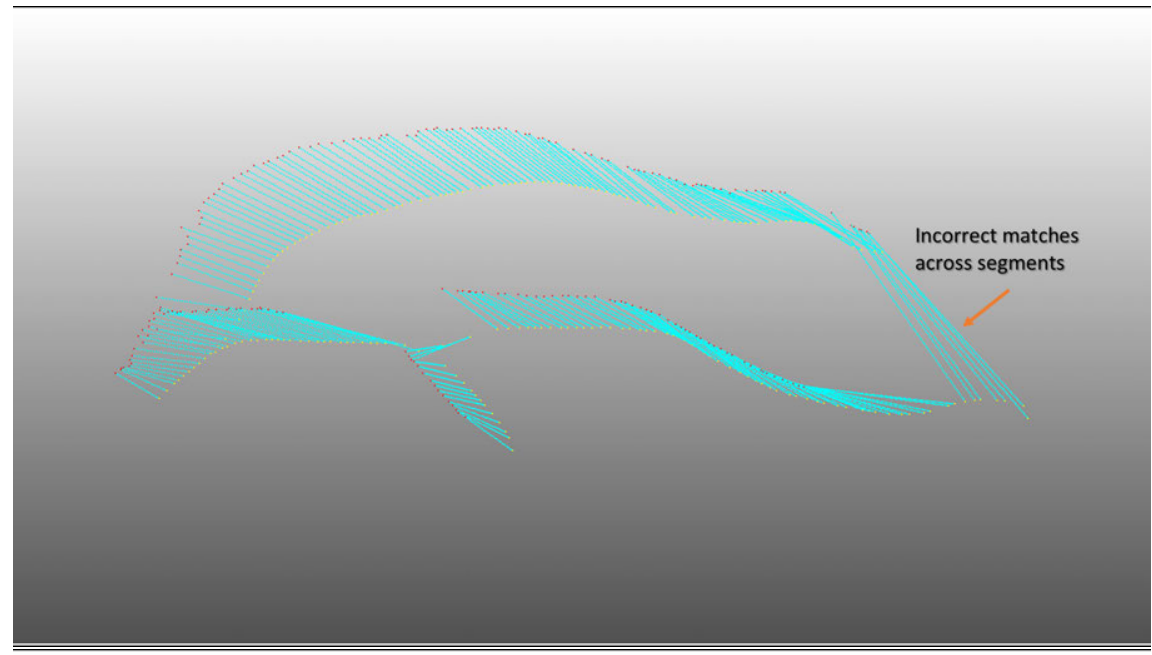

Figure 4.4: Correspondences for the bulging incomplete data case with added noise. At the top are the correspondences only using the CPD and at the bottom the results of the entire method. In the initial case it is possible to see incorrect matches across segments. 


\section{Paper IV}

\section{Methods}
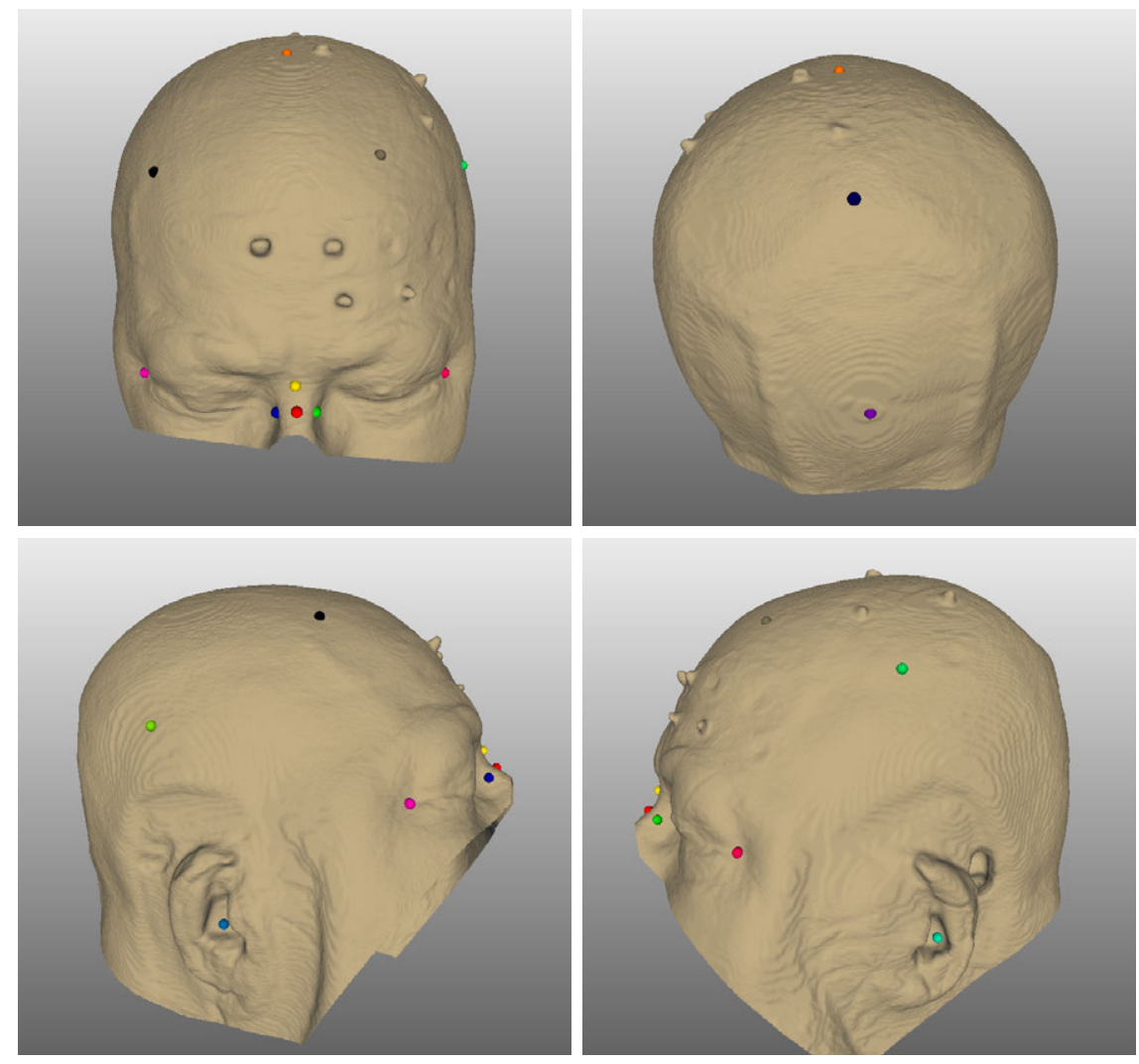

Figure 4.5: Head landmark positions. Each landmark is color coded with a different color.

In this paper we introduced a method for head rigid registration to obtain the rigid transformation between a preoperative dataset and the intraoperative patient position. Fiducial markers are used to align intraoperatively the position of the patient to the preoperative dataset. For the head two main types are used: skin-attached and skull-attached markers. By identifying the marker positions intraoperatively and in the preoperative volume, it is possible to obtain a rigid transformation for alignment. Unfortunately, skin markers suffer from skin shift, which introduces an error in the transformation. Our method aimed at reducing this problem by iteratively searching for a transformation that minimizes the TPS bending energy. It used the fiducial locations (intraoperative and preoperative), points detected on 
the surface of the head (intraoperative - head landmarks, the configuration used in this work is presented in Figure 4.5) plus a segmented MRI head surface and extra points to control the transformation. For evaluation, simulated data was used, where the intraoperative fiducial locations were shifted and points on the head surface were added (targets). Different levels of noise/error were added to the landmarks and their effect on accuracy evaluated.

\section{Results}

The results of our experiment show that our method using an appropriate landmark configuration was able to find the rigid registration solution with a precision of approximately $1 \mathrm{~mm}$ (similar to results of rigidly attached fiducials) if the noise is low (standard deviation $\leq 1 \mathrm{~mm}$ ). For the higher noise level studied (standard deviation $=5 / 3 \mathrm{~mm}$ ) the ME was $2.02 \mathrm{~mm}$ and RMSE $2.46 \mathrm{~mm}$.

\section{Paper V}

\section{Methods}

Once the correspondences between two point sets (landmarks) are known, using methods like the ones presented previously, it is possible to use the TPS to deform a volume dataset and generate a new dataset (Figure 4.6 presents deformation examples). In fact, it is not necessary to generate a new dataset if only rendering is required, wich is named the direct approach. If a new dataset is generated and then rendered it is named the indirect approach.
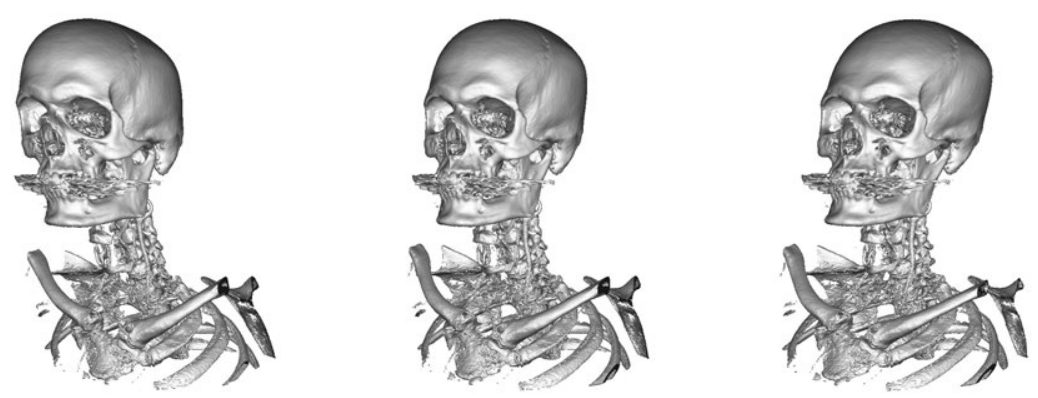

Figure 4.6: Examples of a TPS non-rigid deformation, using a CT of a head and partial chest $(512 \times 512 \times 512)$ dataset. The images on the left and right are deformed and the center one is not deformed.

The aim of this study was to compare the two approaches in terms of performance (speed) and accuracy (image quality). The direct and the indirect approach were carefully implemented to benefit from the massive 
Graphics Processing Unit (GPU) parallel power, using NVIDIA's Compute Unified Device Architecture (CUDA) ${ }^{1}$. They were then tested with CT datasets of varying sizes and with a synthetic image, the Marschner-Lobb function.

\section{Results}

The results show that the direct approach is dependent on the ray sampling steps, number of landmarks and image resolution. The indirect approach is mainly affected by the number of landmarks, if the volume dataset is large enough. These results exclude extreme cases, i.e. in case the sampling steps are much smaller than the voxel size and the image resolution is much higher than the image resolutions used in this study. For a volume of size $512 \times 512 \times 512$, using 100 landmarks and image resolution of $1280 \times 960$, the direct method performs better if the ray sampling steps are approximately above 1 voxel. Regarding accuracy, the direct method provides better results for multiple frequencies using the Marschner-Lobb function.

\subsection{Visualization of Enclosed Objects}

\section{Paper VI}

\section{Methods}

In order to study stereoscopic depth perception of virtual semi-transparent enclosed objects, a stereoscopic display is required. To avoid confounding factors, display types without ghosting artifacts and flickering are preferable. Systems that do not present ghosting and flickering are the dual-monitor single-mirror system originally proposed by Hart [221], and the stereoscopic system with a single monitor also used by Hart. We have developed our own single-monitor-mirror stereoscopic display (Figure 4.7). The stereoscopic display system is composed of one monitor and one acrylic first-surface mirror. The mirror reflects one image for one of the eyes. The geometrical transformations to compute correctly the stereo pair were derived and presented in the paper. System considerations such as mirror placement and implications were also discussed.

In contrast to the similar solutions that use fixed configurations, we tried to optimize the display area by controlling the mirror placement. Consequently, one of the images needed to be skewed. We also developed the rendering engine for DVR of volumetric datasets mostly for medical imaging visualization and using OpenGL for polygonal datasets and stereoscopic digital photography. The skewing process in this case was integrated into the ray-casting of DVR. Using geometrical transformations, we could compute precisely the directions of the rays, producing accurate stereo pairs. A

\footnotetext{
${ }^{1}$ http://www.nvidia.com/object/cuda_home_new.html
} 


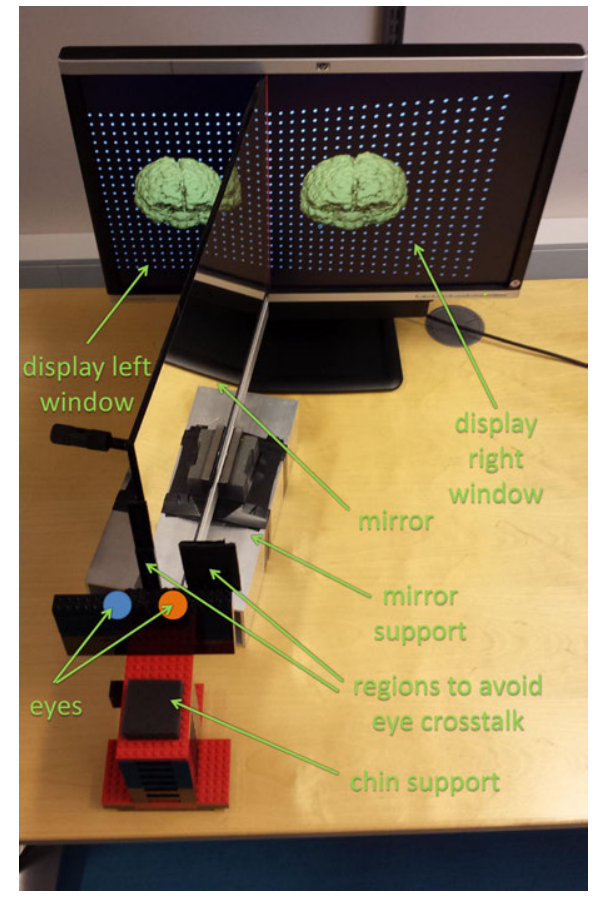

Figure 4.7: Photograph of the stereo system prototype. Included are annotations of the system components and the eye positions.

similar operation was also performed using OpenGL.

\section{Results}

Models that describe the interdependency of the system parameters, when the display area is optimized, were obtained using linear regression. For the data range selected the horizontal left viewing window size increases with the horizontal display and mirror horizontal sizes. The ratio of the horizontal left viewing window size to horizontal display was higher for smaller displays, i.e., a more effective usage of the display is achieved. For larger mirrors, the horizontal left viewing area will approximate half the horizontal display size. The angles of the system field of view in front of the viewing window increase with horizontal display size and decrease with horizontal mirror size. For the field of view behind the viewing window the relation is more complex: the angles increase with horizontal display size, but there seems to be a maximum of the horizontal mirror size for each horizontal display size. Finally, the angles between the horizontal left and reflected right windows increase with horizontal display size and decrease with horizontal mirror size. 


\section{Paper VII}

\section{Methods}

Using the mirror stereoscopic display, presented previously, depth perception experiments were performed. In particular, we investigated how a virtual semi-transparent object (simulating a tumor) is perceived when enclosed in the brain.

Different stereoscopic rendering methods were used to analyze their impact on depth perception accuracy of the enclosed 3D object. The first rendering method used was simple alpha blending with the Blinn-Phong shading model, where a segmented brain (exterior object) and a synthetic tumor (enclosed object) were blended. The second rendering method also used Blinn-Phong, but the shading was modified to preserve silhouettes and to provide an illustrative rendering. Comparing both rendering methods, the brighter regions of the first rendering method will become more transparent in the illustrative rendering method, thus preserving the silhouette areas.

Three depth positions (back, center, front) of the enclosed object were used and the participants had to say where they perceived the object. Figure 4.8 presents lateral views of the enclosed object positions and the brain, using two rendering methods and several opacity values.
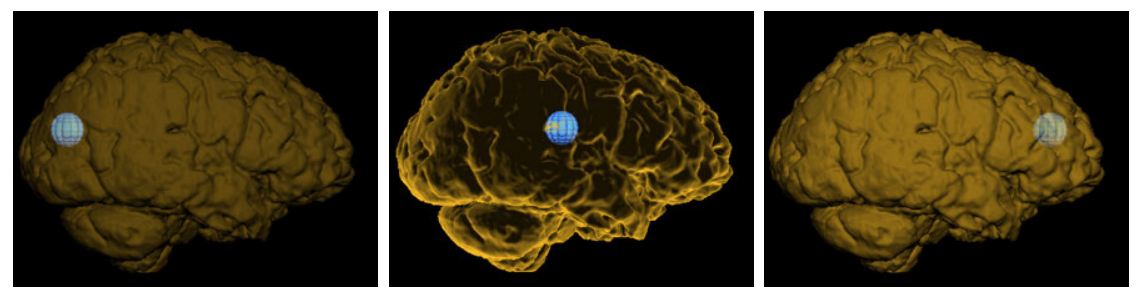

Figure 4.8: Lateral view of the positions where the enclosed object (simulated tumor) is located within the exterior object (brain). Back position (left), center position (middle), front position (right). Two rendering methods were used with different opacity values.

Two experiments were performed: the first tested the hypotheses that depth perception is dependent on the color blending of objects (opacity alpha) for each rendering method and that one of the two rendering methods used is superior. The second experiment was performed to corroborate the results of the first experiment and to test an extra hypothesis: is depth perception improved if an auxiliary object (Figure 4.9) that provides a relationship between the enclosed object and the exterior is used? 


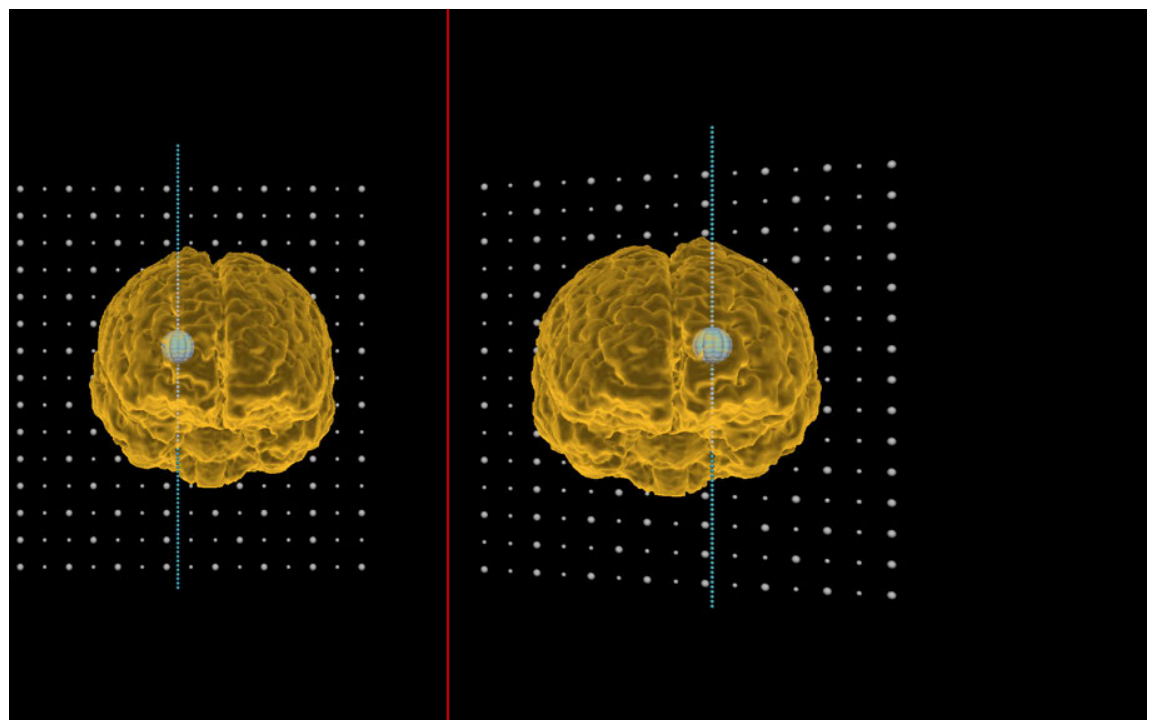

Figure 4.9: Stereo pair used in experiment 2. Illustrative rendering method using the auxiliary object (dotted line).

\section{Results}

The results showed that depth perception accuracy of an enclosed object rendered with a stereoscopic system is dependent on opacity for some rendering methods (simple alpha blending), but this effect is less pronounced than the dependence on object position in relation to the exterior object. The illustrative rendering method is less dependent on opacity. The different rendering methods also performed slightly differently; the illustrative rendering method was superior and the use of an auxiliary object seemed to facilitate depth perception. 


\section{Chapter 5}

\section{Discussion}

\subsection{Optical 3D Reconstruction}

Several optical 3D reconstruction methods have been proposed to capture points on the brain surface (dense or sparse), blood vessels and sulci. The results of our $3 \mathrm{D}$ blood vessel centerlines reconstruction system are similar to other studies using LRS and two-view systems as presented in Table 5.1. The error estimates are approximately $1 \mathrm{~mm}$, which is reasonable for the surgical applications we are considering.

Table 5.1: Summary of optical 3D reconstruction systems accuracy.

\begin{tabular}{|c|c|c|c|}
\hline Study & RMSE & ME & Reconstructed object \\
\hline Jiang et al. 2016[95] & $\begin{array}{l}0.1 \mathrm{~mm}, \\
(\mathrm{TRE}=0.9 \mathrm{~mm})\end{array}$ & - & $\begin{array}{l}\text { phantom surface points (dense). The } \\
\text { TRE was calculated with sparse data. }\end{array}$ \\
\hline Our method (worse case) 2016 & $0.78 \mathrm{~mm}$ & $0.64 \mathrm{~mm}$ & $\begin{array}{l}\text { virtual human brain } 3 \mathrm{D} \text { vessel centeline } \\
\text { points. }\end{array}$ \\
\hline Our method (worse case) 2016 & $1.45 \mathrm{~mm}$ & $1.32 \mathrm{~mm}$ & $\begin{array}{l}\text { phantom } 3 \mathrm{D} \text { vessel centeline points (with } \\
\text { rigid registration). }\end{array}$ \\
\hline Ji et al. 2014[99] & - & $1.05 \mathrm{~mm}$ & phantom surface points (dense). \\
\hline Ji et al. 2014[99] & - & $1.26 \mathrm{~mm}$ & in vivo surface points (dense). \\
\hline Faria et al. 2014[59] & - & $0.6 \mathrm{~mm}$ & phantom surface points (sparse) \\
\hline Pheiffer et al. 2012[159] new LRS & $0.47 \mathrm{~mm}$ & $0.25 \mathrm{~mm}$ & phantom surface points (dense). \\
\hline DeLorenzo et al. 2006[40] & - & $0.78 \mathrm{~mm}$ & $\begin{array}{l}\text { phantom surface points (dense) but the } \\
\text { results were calculated with sparse data. }\end{array}$ \\
\hline DeLorenzo et al. 2006[41] & - & $1.01 \mathrm{~mm}$ & $\begin{array}{l}\text { in vivo surface points (dense) but the re- } \\
\text { sults were calculated with sparse data. } \\
\text { The ground truth points were acquired } \\
\text { with a optically tracked optical pointer. }\end{array}$ \\
\hline Sun et al. 2005[194] & - & $1.16 \mathrm{~mm}$ & in vivo surface points (dense). \\
\hline Sun et al. 2005[194] & - & $0.93 \mathrm{~mm}$ & phantom surface points (dense). \\
\hline Sun et al. 2003[193] & - & $1.6 \mathrm{~mm}$ & in vivo surface points (dense). \\
\hline
\end{tabular}

In some studies, the $3 \mathrm{D}$ superficial blood vessels of the brain were also reconstructed. These either reconstructed first a textured surface using the LRS and then segmented the vessels to obtain the $3 \mathrm{D}$ positions $[23,24]$ or captured an image with a camera, segmented the vessels, registered the camera image to the LRS image and then obtained the 3D positions [45]. Again, the results should be similar to our method.

One important distinction between camera systems and LRS is the acquisition time. Typically camera systems can capture multiple images in one 
second but LRS systems take at least several seconds to acquire the data due to the scanning procedure. This can cause errors because the brain is not an immobile object; instead it pulsates, mainly due to breathing and heart rate. Typically, this is not problematic because the maximum displacement is approximately $1 \mathrm{~mm}$ [59]. Furthermore, the vessel points obtained are not centerline points, but rather points on the outer surface of the vessel. If the vessel is small enough $(\leq 1 \mathrm{~mm})$, then this error can be neglected.

\section{Limitations and Future Research}

Specular highlights and the brain blood vessel topology make the automatic segmentation and extraction of vessel centerlines an extremely difficult problem, although efforts have been made by Ding et al.[46] to produce an automatic method. Moreover, several methods for detection of vessel centerlines exist for the eye (fundus images)[187]. Fundus images are rather different and do not suffer from specular highlights. One possibility to produce images similar to fundus images might be to use ICG fluorescence and near-infrared cameras [196]. This approach should be tested in future studies.

Manual selection of vessels of interest, which proved very effective in our case, has to be further tested in a clinical setting to assess its viability. A benefit of manual segmentation is that a human can easily interpolate small regions of specular highlights, thus reducing this problem.

\subsection{Brain Shift Compensation}

In paper II we have presented our first system/pipeline for non-rigid brain shift compensation. It is comparable to works that also use blood vessel centerlines for registration, such as the work of Reinertsen et al. [166, 168, 167] and Farnia et al.[60] using iUS and Ding et al. [42] using a LRS. Farnia et al. used the same non-rigid registration method as us, the CPD, but with iUS data. The remaining studies used non-rigid registration methods that seemed to produce similar results to the CPD. A comparison between the method used by Ding et al., the TPS-RPM, and the CPD can be found in the article by Myronenko et al. [146].

Although we found a good correlation between the current form of the brain and the virtual deformed volume in our animal study, the CPD and other non-rigid registration methods like the TPS-RPM have some limitations. The main limitation is that the points of the source and target centerlines should be complete. If parts of the vessels are incomplete the method's alignment accuracy can be considerably reduced. Furthermore, in some cases we can observe some incorrect matches across vessel segments that are particularly problematic.

To overcome the previous problems we have developed the method presented in paper III, which combines the CPD with TPS semilandmarks. It has proven to be a robust point-based registration method, with errors below 
$1 \mathrm{~mm}$, even in the presence of noise and incomplete data. These results are better than the CPD alone, in particular when incomplete data is present. To our knowledge, only the method presented by $\mathrm{Hu}$ et al. [89], who used the CPD with landmarks, presents accuracy estimates in the same range as our method, thus landmark information is required to constrain the CPD. One advantage of our method is that the rigid landmarks can be specified beforehand (preoperatively), while Hu's method introduces landmarks e.g. in vessel bifurcation areas during surgery (intraoperatively). Although automatic approaches were suggested to obtain the landmarks, manual selection was performed in their study, suggesting that this procedure is non-trivial. Compared to registration methods that use surface points instead of blood vessels centerlines, the surface to surface registration is less constrained because the problem has more degrees of freedom, thus vessel registration is more robust.

In paper IV our rigid registration method is presented. The results show that by using dense sampling of the search space and the TPS bending energy as the cost function of the optimization method, accurate rigid registration results can be achieved using skin-attached fiducials and landmarks on the surface of the head, with errors similar to those obtained using rigidly attached fiducials if system errors are small.

Once the correspondences between the homologous points are found, then the deformation to compensate for brain shift can be performed, using the TPS in our case. There are two approaches (direct and indirect), studied in paper $\mathrm{V}$. They are compared in terms of performance (speed) and accuracy (image quality).

The advantage of the direct approach is that it does not require the computation of an intermediate volume which is posteriorly rendered; instead, the rendering is performed directly. Fang et al. [58], Westermann et al. [217] and Chen et al. [27] also have implementations that not require the intermediate volume and can use points to control the deformation. Although these seem conceptually similar the main difference is that they use intermediate data structures for acceleration purposes, whereas we rely on brute force GPU acceleration. A better deformed image quality is expected because the previous methods use intermediate data structures for approximation purposes to trade image quality for speed. The most similar method to ours is the free-form method by Chen et al. [27], but a key difference is that the free-form method deforms the rays in the original volume space and then traverses them. In our approach the rays are straight and traversed in the deformed volume space; the sampling positions are backward mapped to obtain the intensity values in the original volume space.

Comparing the two approaches in terms of performance, we note that the indirect method is less sensitive to changes in the ray-sampling steps and to image resolution, while both seem to be similarly affected by the number of landmarks. For larger volumes and greater sampling steps, the performance of the indirect method is worse than the direct method. There 
are also other drawbacks of the indirect method, for instance the inability to trade image quality for frame rate, because the main parameter used for this purpose is the ray-sampling steps and for large volumes the indirect method presents almost no change (constant). In contrast, the direct method is very sensitive to the ray-sampling steps, thus allowing easy control of the frame rate. Moreover, the indirect method requires that two volumes are loaded simultaneously to texture memory, which for larger volumes can be a serious limitation.

To measure the accuracy of both approaches the image quality metrics Peak Signal-to-Noise Ratio (PSNR) and the Structural Similarity index (SSIM) [214] were used. The direct method gave superior results, in particular when an implicit function was used. The worse results of the indirect method can be explained by the double trilinear interpolation: first at the evaluation of each voxel value (backward mapping - generation of intermediate volume) and second in the reconstruction (at each sampling position). Furthermore, the results of the indirect method also depend on the resolution with which the intermediate volume is sampled. The direct implicit method is also less sensitive to frequency, because no volume grid sampling is performed to obtain the voxel intensities. In this case, the sources of errors reside in the sampling positions along the ray and in numerical rounding errors in the TPS calculation. However, these are rather small errors.

\section{Limitations and Future Research}

In some cases it is not possible to identify large vessels in some regions of the exposed brain surface because they are not present or are occluded. To overcome this problem, in the future we would like to combine our method with methods that use surface points.

The method presented in paper III is generic in the sense that it does not require that the vessel centerlines lie on a surface. Such data is often generated by ultrasound, thus our method can also be applied to this type of data. This opens the possibility to combine information about the brain surface and the interior. Considerable effort was made to correct for incorrect vessel correspondences (particularly, incorrect matches/correspondences across segments), yet it is still unclear the maximum amount of initial incorrect correspondences tolerable.

Besides the errors analyzed for the rigid registration in paper IV, there are other sources of errors that impact the method. The first is the resolution of the preoperative MRI dataset. This will affect the quality of the segmented surface and the size of each triangle that belongs to the surface. Since our method projects the landmark points to the closest triangle surface, this step introduces an error that depends on the triangle size. In our case, the resolution of the MRI dataset is $0.5 \times 0.5 \times 0.6 \mathrm{~mm}$, producing triangles with edges approximately the same size, and thus the minimum error is also in this range. The second source of error is related to the optimization. Even though we use dense sampling of the search space (many 
particles/transformations) it is still possible for the optimization to get stuck in local minima, particularly when the search is near the optimum. This was the motivation for using a dense sampling space instead of optimization techniques such as simulated annealing [112], which in our experiments always got stuck in a local minimum far from the global solution. Finally, we used fixed sampling steps for the neighbors in the optimization process. This can also introduce a small error.

The rigid registration method relies on the assumption that the shape of the head does not change considerably. For the areas where we have placed the fiducials, this is typically the case. But in certain areas such as the region around the ears which is compressed by the MRI headset a deformation will happen. This deformation should not be larger than the maximum scalp thickness, which for humans is approximately $5 \mathrm{~mm}$ [87]. For this reason the maximum error level selected in this study has a standard deviation of $5 / 3 \mathrm{~mm}$, considering that according to the three standard deviation rule the maximum error is around $5 \mathrm{~mm}(99.7 \%)$.

As the results show, in these cases the accuracy of the method decreases, but still it is possible to achieve a solution that minimizes the bending energy. The decision on whether it is a valid solution will depend on visual inspection relating to the available anatomical landmarks. Anatomical landmarks on the nose and near the ear canal are rather important because the skin thickness is much lower than in the remaining locations, minimizing the risk of shape change.

In the rigid registration study only one landmark configuration was used, but other configurations are possible. Our recommendation is to use at least three anatomical landmarks in order to visually inspect incorrect solutions. The main reason the method fails to produce a correct solution is the close to spherical shape of the head, which produces many solutions with low bending energy. If an incorrect solution is found, in order to disambiguate and remove it from the space of all possible solutions more landmarks must be selected on the head or a better landmark configuration must be provided.

In paper $\mathrm{V}$ we did not explore faster deformation techniques, as we were interested in preserving image quality and keeping the method as general as possible. Fast RBF alternatives that trade image quality for speed, are presented by Rowland [171] and Lapeer et al. [122]. It is also possible to use the locally bounded Hardy method [58], and RBF with compact support [70]. The choice of the method to be used depends on the data and the purpose. Our implementation can easily be changed to use some of these methods, if necessary.

In the future we would like to combine our vessel registration method with surface registration. Furthermore, we would like to combine information of the brain surface (using a camera system) with information of the brain interior (using iUS). Finally, we would also like to compare and validate the results of the TPS deformations against biomechanical models and iMRI. 


\subsection{Visualization for Brain Tumor Surgery}

Papers VI and VII present our stereoscopic single-monitor display system and the perception study conducted using it. The goal of the study was to test how humans perceive the enclosed object position in relation to an exterior object, by varying rendering methods, opacity values and the enclosed object depth position. The study was designed for neurosurgery applications, as it is common that the tumor is enclosed in the brain.

The previous reported single-monitor system ${ }^{1}$ was not designed for DVR and polygonal rendering. It is technically possible to compute the correct stereo pairs for this fixed configuration, but in this case, the position of the eyes must be controlled, thus increasing the system implementation difficulty, because the eyes are constrained by the fixed mirror position. We have focused our attention on producing accurate stereo pairs. But there are situations when it is enough to have an approximation as with the previous system.

Evaluations of medical applications using stereoscopic systems have been performed. Some even go back as far as 1990, e.g. Owczarczyk et al. [156], whereas a more recent study performed by radiologists comparing different $3 \mathrm{D}$ systems can be found in [198]. Several works in the visualization literature suggest that by using stereopsis, we greatly increase the understanding of depth [91, 90], in particular for medical datasets [108]. However, it should, be pointed out that the usage of stereopsis is very task dependent, as shown by Wickens et al. [218].

The results of our perception study showed that the illustrative rendering method was superior to the simple alpha blending rendering method for depth perception position accuracy of enclosed objects visualized using our stereoscopic system. Although both methods use the Blinn-Phong shading model, the shading of the illustrative rendering method was modified to preserve the silhouette areas (shape information) and deemphasize the remaining areas. These modifications impacted significantly on the performance of the rendering method.

For the simple alpha blending rendering method, depth perception depends on opacity, with a stronger dependence if the tumor is further away (increasing depth) from the brain. The results suggest that the tumor position has a stronger influence than opacity in depth perception. Considering all positions, the optimal opacity value for the tumor seems to be around 0.6 , found by fitting a quadratic model to the data. It should be pointed out that a quadratic model was not expected a priori, but since the appearance of several of the graphs indicated that there is an optimum at an intermediate opacity value rather than at one of the extremes, we wanted to apply a model that allowed for such an optimum.

For the illustrative rendering method, the results indicate that there is no relation between depth perception and opacity in the opacity range tested.

\footnotetext{
${ }^{1}$ http://stereo.jpn.org/eng/stphmkr/mirror/mirrorview.htm
} 
The closest position of the tumor in relation to the observer is significantly better than the remaining positions. But it is not clear whether there is a linear dependency of depth perception accuracy and the position of the enclosed object.

A significant difference in accuracy for both rendering methods was found when using normalized opacity values. The original opacities (alpha) generate non-comparable representations for both rendering methods due to the extra transformations of the illustrative rendering method. For this reason the normalization was required in order to study the same range of values.

The motivation behind the use of the selected rendering methods is their simplicity, allowing iterative frame rates and their ability to preserve to some extent important shape features that may relate to anatomical locations (considering the brain - gyri and sulci) used by surgeons for navigation purposes. These important features may be deemphasized if other visualization methods using points or wireframe are considered.

The results also indicate that the use of an auxiliary object increases depth perception accuracy. When the auxiliary object is used with both rendering methods the differences are significant, with the illustrative rendering method providing better accuracy. These relationships are clearer for positions further away. If the enclosed object is placed near the surface of the exterior object then there is no need to use the auxiliary object and either of the rendering methods is appropriate.

A previous study by Maurer et al. [137] comparing rendering methods, reported that if the enclosed object was rendered with wire-frame or texturemapped dot patterns, the depth perception accuracy increased. These illustrative rendering methods are closely related to our illustrative rendering method, which also provided better accuracy in our experiments. The difference is that in our case the object rendered with the illustrative method was the exterior object, not the enclosed object. It seems that illustrative rendering methods provide added value in depth perception accuracy, and similar results were found for shape perception where the exterior object was enhanced with texture [93].

\section{Limitations and Future Research}

Although our analysis was designed to focus only on the relationships between the factors under study and depth perception accuracy, there might still be confounding factors not accounted for. Shape may be one of them, although an attempt was made to provide complex shapes for the analysis. The correct head position of the participant may also slightly influence the results. Considering the strong dependencies found, we believe that these confounding factors, if present, are minimal.

With the simple alpha blending rendering method, it was noticed that the optimal opacity value was higher for the enclosed object (0.62) than for the exterior object $(1-0.62)$. Since we are using the same values of the base color luminance, this implies that the luminance of each surface is 
mainly dependent on opacity (shading also plays a role). In the optimal case, the luminance value is higher for the enclosed object than for the exterior surface. Traditionally for monoscopic rendering using perception models, the reverse relation is used to aid in depth ordering, i.e. the closer surface to the observer has a higher luminance [228]. This is a clearly ambiguous phenomenon that should be further studied.

In the future, we want to study depth perception accuracy with more than two objects (or two surface intersections) and not only limited to one enclosed object. This relates to the more general situation found in DVR. Ultimately we want to find guidelines for stereoscopic DVR in order to obtain high depth perception accuracy.

Although our stereoscopic display system and the perception study have interesting features, they are probably not suitable for use in the OR, mainly due to the large size of the display. Instead, we envision the stereoscopic display use as a preoperative planning or training tool, where it would be used to help in understanding the position of the objects of interest in relation to each other and perhaps to plan the path to access the tumor.

To visualize the tumor position intraoperatively a number of solutions are possible, for example as we have used in our perception study with a segmented 3D brain and tumor (virtual), other solutions combine 3D information with $2 \mathrm{D}$ and it is also possible to use augmented reality solutions. For neurosurgery there is even an augmented reality system, known as MAGI $[51,53,52]$, which fuses virtual objects with microscope images. The virtual images are overlaid directly with the surgical microscope view. But it is still unclear what is the best visualization solution (virtual or augmented reality), rendering method and display technology (microscope, mono or stereoscopic displays) to aid the surgeon during the operation. If augmented reality solutions are used it is also unclear what is the best rendering method to blend the virtual and real objects, and still perceive the positions between these objects correctly. A possible scenario is that we want to blend two images: a virtual tumor image (segmented from the MRI) and an intraoperative brain surface image captured by a camera. Considering this scenario, what parameters values and rendering methods should be used for the blending to be effective? Such questions should be addressed in the future, and open possibilities for interesting visualization research.

\subsection{Reflections}

Considering that all the registration, segmentation and visualization problems have been solved and that the TPS or a biomechanical model are appropriate to deform the preoperative dataset to mimic the brain movement; then it will be possible to present an accurate representation of the anatomy. This information could be used in combination with fluorescence techniques as a navigation aid. In cases where it is not possible to use fluorescence (such as low grade gliomas) then our technique could be used to identify the 
tumor area and eloquent areas. Besides our methods, the main technical limitation is related to image resolution, particularly if fMRI, which has a rather poor image resolution, is used to identify the eloquent areas, causing segmentation errors. Typically, neurosurgeons resect the brain tumor with a margin to compensate for these errors, unless they are close to an eloquent area. Even if the eloquent area segmentation is poor it provides information about the approximate region, and further tests might be performed in this region, such as: navigated transcranial magnetic stimulation, intraoperative neurophysiology and clinical neurological testing in awake patients. One drawback of our method is that the deformation mainly uses brain surface data. Considering only the eloquent areas, most of these are related to the brain cortex. The accuracy at the brain surface is higher, thus at least a good spatial representation of the regions to preserve can be achieved.

Although we have focused on the application of our methods for brain tumor resection, they probably have applications for other neurosurgical pathologies such as vascular and epilepsy neurosurgery. Regarding epilepsy, DeLorenzo et al. [39] have already proposed the use of a similar method to ours to address this problem. Epilepsy surgery procedures such as cortical excision and hemispherectomy that involve resection of the brain cortex should be of particular interest due to the reasons discussed previously. For vascular surgery there have been also interesting developments using augmented reality, as reported by Kersten-Oertel et al. [109, 110, 111]. 


\section{Chapter 6}

\section{Conclusions}

In this work we have created methods and tools for brain tumor resection surgery. The focus was on brain shift compensation, including vessel reconstruction, registration and volume deformation. Aspects of visualization, in particular how the position of a tumor is perceived when enclosed in the brain, were also studied. The following conclusions can be drawn:

- In paper I was developed and evaluated a new method for 3D superficial vessel centerline reconstruction using a multi-view camera system and methods that use the geometrical properties of the vessel structures. The error estimates were approximately $1 \mathrm{~mm}$, which is reasonable for the surgical applications we are considering;

- In papers II and III were developed/applied and evaluated non-rigid registration methods to find the correspondences between vessel centerlines of preoperative and intraoperative data. Our novel method presented in paper III combining the CPD with TPS semilandmarks produced errors well below $1 \mathrm{~mm}$, even in the presence of noise and incomplete data. Again, these values are reasonable for the surgical applications we are considering, i.e. brain shift compensation for brain tumor removal;

- In paper IV was developed and evaluated a rigid registration method to align the intraoperative patient position with the preoperative MRI dataset. The results showed that our rigid registration method using skin-attached fiducials and head landmarks was precise to approximately $1 \mathrm{~mm}$ (similar to results of rigidly attached fiducials), given that the localization errors are low (standard deviation $\leq 1 \mathrm{~mm}$ ). The accuracy decreases linearly with the increase of the localization error. In extreme cases it is necessary to include more head landmarks to find an appropriate registration solution;

- In paper $\mathrm{V}$ were developed and evaluated methods for fast volume deformation to compensate for brain shift, using the TPS (direct and 
indirect approaches) and the correspondences found by registration methods. The comparisons of both approaches were made in terms of performance and accuracy. Regarding performance, the indirect method is superior if the sampling along the rays is high, in comparison to the voxel grid, while the direct method is superior otherwise. The accuracy analysis seemed to indicate that the direct method is superior, in particular when the implicit function is used;

- In papers VI and VII was presented a new stereoscopic display and conducted perception studies with this display. Comparing our stereoscopic display to the most similar systems, the dual and single-monitor systems, the main difference is its flexible configuration, which can be used to exploit effectively the display area. We also developed rendering engines for DVR of volumetric datasets, mostly for medical imaging visualization, polygonal datasets using OpenGL, and digital photographs, in a geometrically correct way. In spite of certain geometrical constraints, the system might prove useful for medical imaging applications.

The perception study investigated how the position of a tumor is perceived in relation to the brain, when the tumor is enclosed in the brain. In this study, the stereoscopic display and different rendering methods, object positions and color blending (opacity) values, were used. The study showed that depth perception accuracy is dependent on opacity for some rendering methods (simple alpha blending), but this effect is less pronounced than the dependence on object position in relation to the exterior object. The different rendering methods also performed slightly differently; an illustrative rendering method was superior and the use of an auxiliary object seemed to facilitate depth perception. 


\section{Bibliography}

[1] K. Abhari, J. S. H. Baxter, E. C. S. Chen, A. R. Khan, T. M. Peters, S. de Ribaupierre, and R. Eagleson. Training for Planning Tumour Resection: Augmented Reality and Human Factors. IEEE Transactions on Biomedical Engineering, 62(6):1466-1477, 2015.

[2] A. Ahmadian, N. S. Dadashi, S. Karimifard, and P. Farnia. An efficient method for estimation of soft tissue deformation based on intraoperative stereo image features and point-based registration. International Journal of Imaging Systems and Technology, 23(4):294-303, 2013.

[3] R. A. Akerstrom and J. T. Todd. The perception of stereoscopic transparency. Perception \& Psychophysics, 44(5):421-432, 1988.

[4] U. E. Aladl and T.Peters. Multi Modality State-of-the-Art Medical Image Segmentation and Registration Methodologies, volume 2, chapter Medical Image Registration. Springer, 2011.

[5] J. T. Alander, I. Kaartinen, A. Laakso, T. Pätilä, T. Spillmann, V. V. Tuchin, M. Venermo, and P. Välisuo. A review of indocyanine green fluorescent imaging in surgery. International Journal of Biomedical Imaging, 2012:1-26, 2012.

[6] N. Archip, O. Clatz, S. Whalen, D. Kacher, A. Fedorov, A. Kot, N. Chrisochoides, F. Jolesz, A. Golby, P. M. Black, and S. K. Warfield. Non-rigid alignment of pre-operative MRI, fMRI, and DT-MRI with intra-operative MRI for enhanced visualization and navigation in image-guided neurosurgery. NeuroImage, 35(2):609-624, 2007.

[7] K. S. Arun, T. S. Huang, and S. D. Blostein. Least-squares fitting of two 3-D point sets. IEEE Transactions on Pattern Analysis and Machine Intelligence, 9:698-700, 1987.

[8] C. Askeland, O. V. Solberg, J. B. L. Bakeng, I. Reinertsen, G. A. Tangen, E. F. Hofstad, D. H. Iversen, C. Våpenstad, T. Selbekk, T. Langø, T. A. N. Hernes, H. O. Leira, G. Unsgård, and F. Lindseth. Custusx: 
an open-source research platform for image-guided therapy. International Journal of Computer Assisted Radiology and Surgery, pages $1-15,2015$.

[9] M. A. Audette, K. Siddiqi, F. P. Ferrie, and T. M. Peters. An integrated range-sensing, segmentation and registration framework for the characterization of intra-surgical brain deformations in image-guided surgery. Computer Vision and Image Understanding, 89:226-251.

[10] Barco. Stereoscopic projection: 3d projection technology, 2003.

[11] Z. Bardosi and W. Freysinger. Estimating FLE $E_{\text {image }}$ distributions of manual fiducial localization in CT images. International Journal of Computer Assisted Radiology and Surgery, 2016.

[12] B. Bellekens, V. Spruyt, and M. Weyn. A Survey of Rigid 3D Pointcloud Registration Algorithms. In AMBIENT 2014, The Fourth International Conference on Ambient Computing, Applications, Services and Technologies, pages 8-13. IARIA, 2014.

[13] B. Berkels, I. Cabrilo, S. Haller, M. Rumpf, and K. Schaller. Coregistration of intra-operative brain surface photographs and preoperative mr images. International Journal of Computer Assisted Radiology and Surgery, 9(3):387-400, 2014.

[14] A. Berthier. Images stéréoscopiques de grand format (in French). Cosmos, 34(590 and 591):205-210 and 227-233, May 1896.

[15] P. J. Besl and N. D. Mckay. A Method for Registration of 3-D Shapes. IEEE Transactions on Pattern Analysis and machine Intelligence, 14(2):239-256, 1992.

[16] G. Bianchi. Exploration of Augmented Reality Technology for Surgical Training Simulators. PhD thesis, Swiss Federal Institute of Technology Zurich, 2006.

[17] F. L. Bookstein. Landmark methods for forms without landmarks: Morphometrics of group differences in outline shape. Medical Image Analysis, 1(3):225-243, 1997.

[18] F.L. Bookstein. Principal Warps: Thin-Plate Splines and the Decomposition of Deformations. IEEE Transactions on Pattern Analysis and Machine Intelligence, 11:567-585, 1989.

[19] C. Boucheny, G. Bonneau, J. Droulez, G. Thibault, and S. Ploix. A Perceptive Evaluation of Volume Rendering Techniques. ACM Transactions on Applied Perception (TAP), 5(4), January 2009.

[20] G. Bradski. The OpenCV Library. Dr. Dobb's Journal of Software Tools, 2000. 
[21] M. Brydegaard, N. Haj-Hosseini, K. Wårdell, and S. AnderssonEngels. Photobleaching-Insensitive Fluorescence Diagnostics in Skin and Brain Tissue. IEEE Photonics Journal, 3(3):407-421, 2011.

[22] R. D. Buchholz, D. D. Yeh, J. W. Trobaugh, L. L. McDurmont, C. D. Sturm, C. Baumann, J. M. Henderson, A. Levy, and P. Kessman. The correction of stereotactic inaccuracy caused by brain shift using an intraoperative ultrasound device. In First Joint Conference Computer Vision, Virtual Reality and Robotics in Medicine and Medial Robotics and Computer-Assisted Surgery, pages 19-22, 1997.

[23] A. Cao, M. I. Miga, P. Dumpuri, S. Ding, B. M. Dawant, and R. C. Thompson. Target error for image-to-physical space registration: preliminary clinical results using laser range scanning. In Medical Imaging 200\%: Visualization and Image-Guided Procedures, volume 6509. SPIE, 2007.

[24] A. Cao, R. C. Thompson, P. Dumpuri, B. M. Dawant, R. L. Galloway, S. Ding, and M. I. Miga. Laser range scanning for image-guided neurosurgery: Investigation of image-to-physical space registrations. Medical Physics, 35(4):1593-1605, 2008.

[25] T. Chai and R. R. Draxler. Root mean square error (RMSE) or mean absolute error (MAE)? - Arguments against avoiding RMSE in the literature. Geoscientific Model Development, 7:1247-1250, 2014.

[26] M. Chan, Y. Wu, W. Mak, W. Chen, and H. Qu. PerceptionBased Transparency Optimization for Direct Volume Rendering. IEEE Transactions on Visualization and Computer Graphics, 15(6):12831290, November/December 2009.

[27] H. Chen, J. Hesser, and R. Manner. Raycasting free-form deformedvolume objects. The Journal of Visualization and Computer Animation, 14:61-72, 2003.

[28] I. Chen, A. M. Coffey, S. Ding, P. Dumpuri, B. M. Dawant, R. C Thompson, and M. I. Miga. Intraoperative brain shift compensation: Accounting for dural septa. IEEE Transactions on Biomedical Engineering, 58(3).

[29] S. J. Chen, I. Reinertsen, P. Coupé, C. X. Yan, L. Mercier, D. R. Del Maestro, and D. L. Collins. Validation of a hybrid doppler ultrasound vessel-based registration algorithm for neurosurgery. International Journal of Computer Assisted Radiology and Surgery, 7(5):667-685, 2012.

[30] Y. Chen and G. Medioni. Object Modeling by Registration of Multiple Range Images. In 1991 IEEE International Conference on Robotics and Automation, pages 2724-2729, 1991. 
[31] H. Chui and A. Rangarajan. A new point matching algorithm for non-rigid registration. Journal Computer Vision and Image Understanding, 89(2-3):114-141, 2003.

[32] O. Clatz, H. Delingette, I. F. Talos, A. J. Golby, R. Kikinis, F. A. Jolesz, N. Ayache, and S. K. Warfield. Robust Nonrigid Registration to Capture Brain Shift From Intraoperative MRI. IEEE Transactions on Medical Imaging, 24(11):1417-1427, 2005.

[33] D. Collins and A. Evans. ANIMAL: Validation and application of nonlinear registration-based segmentation. In IEEE International Symposium on Biomedical Imaging: From Nano to Macro, volume 11, 1997.

[34] ACR-NEMA Commitee. Digital Imaging and Communications, 1985. ACR-NEMA Standards Publication 300-1985 Washinton, DC: National Electrical Manufactures Association.

[35] J. E. Cutting and P. M. Vishton. Perceiving layout and knowing distances: The integration, relative potency, and contextual use of different information about depth. "W. Epstein and S. Rogers (eds.), Handbook of perception and cognition", 1995.

[36] M. J. Daly, J.H. Siewerdsen, D.J. Moseley, D.A. Jaffray, and J.C. Irish. Intraoperative cone-beam CT for guidance of head and neck surgery: Assessment of dose and image quality using a C-arm prototype. Medical Physics, 33(10):3767-3780, 2006.

[37] N. D'Apuzzo and M. Verius. 3D Monitoring of the Intraoperative Brainshift by Means of Photogrammetry. In Proceedings SPIE: ThreeDimensional Image Capture and Applications 2008, 6805, 2008.

[38] R. Dashti, J. Hernesniemi, and M. Niemela. The role of intra operative Indocyanine green video angiography in cerebrovascular surgery. European Neurological Review, 2:11-12, 2007.

[39] C. DeLorenzo, X. Papademetris, L. H. Staib, K. P. Vives, D. D. Spencer, and J. S. Duncan. Image-Guided Intraoperative Cortical Deformation Recovery Using Game Theory: Application to Neocortical Epilepsy Surgery. IEEE Transactions on Medical Imaging, 29(2):322338, 2010.

[40] C. DeLorenzo, X. Papademetris, K. Wu, K. P. Vives, D. Spencer, and J. S. Duncan. Combined feature/intensity-based brain shift compensation using stereo guidance. In IEEE International Symposium on Biomedical Imaging: From Nano to Macro, pages 335-338. IEEE, 2006.

[41] C. DeLorenzo, X. Papademetris, K. Wu, K. P. Vives, D. Spencer, and J. S. Duncan. Nonrigid 3D brain registration using intensity/feature 
information. In Medical Image Computing and Computer-Assisted Intervention - MICCAI 2016, Lecture Notes in Computer Science, 4190, pages 932-939, 2006.

[42] S. Ding, M. I. Miga, J. H. Noble, A. Cao, P. Dumpuri, R. C. Thompson, and B. M. Dawant. Semiautomatic Registration of Pre- and Postbrain Tumor Resection Laser Range Data: Method and Validation. IEEE Transactions on Biomedical Engineering, 56(3):770-780, 2009.

[43] S. Ding, M. I. Miga, T. S. Pheiffer, A. L. Simpson, R. C. Thompson, and B. M. Dawant. Tracking of Vessels in Intra-Operative Microscope Video Sequences for Cortical Displacement Estimation. IEEE Transactions on Biomedical Engineering, 58(7):1985-1993, 2011.

[44] S. Ding, M. I. Miga, R. C. Thompson, and B. M. Dawant. Robust vessel registration and tracking of microscope video images in tumor resection neurosurgery. In IEEE International Symposium on Biomedical Imaging: From Nano to Macro, 2009., pages 1043-1046. IEEE, 2009.

[45] S. Ding, M. I. Miga, R. C. Thompson, P. Dumpuri, A. Cao, and B. M. Dawant. Estimation of intra-operative brain shift using a tracked laser range scanner. In 29th Annual International Conference of the IEEE. Engineering in Medicine and Biology Society, 2007., pages 848-851. IEEE, 2007.

[46] S. Ding, M. I. Miga, R. C. Thompson, I. Garg, and B. M. Dawant. Automatic segmentation of cortical vessels in pre- and post-tumor resection laser range scan images. In Medical Imaging 2009: Visualization, Image-Guided Procedures, and Modeling, volume 7261. SPIE, 2009.

[47] F. Drakopoulosa and N. P. Chrisochoidesa. Accurate and fast deformable medical image registration for brain tumor resection using image-guided neurosurgery. Computer Methods in Biomechanics and Biomedical Engineering: Imaging \& Visualization, 4(2):112-126, 2016.

[48] R. A. Drebin, L. Carpenter, and P. Hanrahan. Volume rendering. ACM SIGGRAPH Computer Graphics, 22(4):65-74, 1988.

[49] A. Drevelegas and N. Papanikolaou. Imaging of Brain Tumors with Histological Correlations, chapter Imaging Modalities in Brain Tumors. Springer, 2011.

[50] V. Duay, T. K. Sinha, P. D'Haese, M. I. Miga, and B. M. Dawant. Non-rigid Registration of Serial Intra-operative Images for Automatic Brain Shift Estimation. In Biomedical Image Registration, Lecture Notes in Computer Science, 2717, pages 61-70, 2003. 
[51] P. J. Edwards, D. J. Hawkes, D. L. G. Hill, D. Jewell, R. Spink, A. J. Strong, and M. J. Gleeson. Augmentation of Reality Using an Operation Microscope for Otolaryngology and Neurosurgical Guidance. Journal of Image Guided Surgery, 1(3):172-178, 1995.

[52] P. J. Edwards, L. G. Johnson, D. J. Hawkes, M. R. Fenlon, A. J. Strong, and M. J. Gleeson. Clinical Experience and Perception in Stereo Augmented Reality Surgical Navigation. In Medical Image and Augmented Reality - MIAR 2004, Lecture Notes in Computer Science, 3150, pages 369-376, 2004.

[53] P. J. Edwards, A. P. King, C. R. Maurer Jr., D. A. de Cunha, D. J. Hawkes, D. L. G. Hill, R. P. Gaston, M. R. Fenlon, S. Chandra, A. J. Strong, C. L. Chandler, A. Richards, and M. J. Gleeson. Design and Evaluation of a System for Microscope-Assisted Guided Interventions (MAGI). IEEE Transactions on Medical Imaging, 19(1):1082-1093, 2000 .

[54] A. Elnakib, G. Gimel'farb, J. S. Suri, and A. El-Baz. Multi Modality State-of-the-Art Medical Image Segmentation and Registration Methodologies, volume 2, chapter Medical Image Segmentation: A Brief Survey. Springer, 2011.

[55] D. J. Engle and L. D. Lunsford. Brain tumor resection guided by intraoperative computed tomography. Journal of Neuro-Oncology, 4(4):361-370, 1987.

[56] A. Enquobahrie, P. Cheng, K. Gary, L. Ibanez, D. Gobbi, F. Lindseth, Z. Yaniv, S. Aylward, J. Jomier, and K. Cleary. The Image-Guided Surgery Toolkit IGSTK: An Open Source C++ Software Toolkit. Journal of Digital Imaging, 20(1):21-33, 2007.

[57] X. Fan, S. Ji, A. Hartov, D. W. Roberts, and K. D. Paulsen. Stereovision to MR image registration for cortical surface displacement mapping to enhance image-guided neurosurgery. Medical Physics, 41(10), 2014.

[58] S. Fang, R. Srinivasan, R. Raghavan, and J. T. Richtsmeier. Volume morphing and rendering - an integrated approach. Computer Aided Geometric Design, 17:59-81, 2000.

[59] C. Faria, O. Sadowsky, E. Bicho, G. Ferrigno, L. Joskowicz, M. Shoham, R. Vivanti, and E. De Momi. Validation of a stereo camera system to quantify brain deformation due to breathing and pulsatility. Medical Physics, 41(11), 2014.

[60] P. Farnia, A. Ahmadian, A. Khoshnevisan, A. Jaberzadeh, S. N Dadashi, and A. F. Kazerooni. An efficient point based registration of intra-operative ultrasound images with MR images for computation of 
brain shift; A phantom study. In Proceedings of IEEE conference on engineering in medicine and biology (EMBS 2011), pages 8074-8077. IEEE, 2011.

[61] P. Farnia, A. Ahmadian, T. Shabanian, N. D. Serej, and J. Alirezaie. Brain-shift compensation by non-rigid registration of intra-operative ultrasound images with preoperative MR images based on residual complexity. International Journal of Computer Assisted Radiology and Surgery, 10(5):555-562, 2015.

[62] A. Fedorov, E. Billet, M. Prastawa, G. Gerig, A. Radmanesh, S.K. Warfield, R. Kikinis, and N. Chrisochoides. Evaluation of Brain MRI Alignment with the Robust Hausdorff Distance Measures. In ISVC 2008, Part I, LNCS 5358, pages 594-603. Springer-Verlag, 2008.

[63] A. Fedorov, Beichel R., J. Kalpathy-Cramer, J. Finet, J-C. FillionRobin, S. Pujol, C. Bauer, D. Jennings, F. Fennessy, M. Sonka, J. Buatti, S. R. Aylward, J. V. Miller, S. Pieper, and R. Kikinis. 3D Slicer as an Image Computing Platform for the Quantitative Imaging Network. Magnetic Resonance Imaging, 30(9):1323-1341, 2012.

[64] A. J. Ferenc. Intraoperative Imaging and Image-Guided Therapy. Springer, 2014.

[65] J. Fergason, S. Robinson, C. McLaughlin, B. Brown, A. Abileah, T. Baker, and P. Green. An innovative beamsplitter-based stereoscopic/3D display design. SPIE Stereoscopic Displays and Virtual Reality Systems, 5664:488-494, May 2005.

[66] J. M. Fitzpatrick. Fiducial registration error and target registration error are uncorrelated. In Medical Imaging 2009: Visualization, ImageGuided Procedures, and Modeling, volume 7261. SPIE, 2009.

[67] J. M. Fitzpatrick, J. West, and C. R. Maurer. Derivation of expected registration error for rigid-body, point-based image registration. In Medical Imaging 1998: Image Processing, volume 3338, pages 16-27. SPIE, 2009.

[68] J. M. Fitzpatrick and J. B. West. The distribution of target registration error in rigid-body point-based registration. IEEE Transactions on Medical Imaging, 20(9):917-927, 2001.

[69] J. M. Fitzpatrick, J. B. West, and C. R. Maurer. Predicting error in rigid-body point-based registration. IEEE Transactions on Medical Imaging, 17(5):694-702, 1998.

[70] M. Fornefett, K. Rohr, and H.S. Stiehl. Radial basis functions with compact support for elastic registration of medical images. Image and Vision Computing, 19:87-96, 2001. 
[71] A. F. Frangi, W. J. Niessen, K. L. Vincken, and M. A. Viergever. Multiscale vessel enhancement filtering. In Medical Image Computing and Computer-Assisted Intervention - MICCAI'98, Lecture Notes in Computer Science, 1496, pages 130-137, 1998.

[72] B. Froner. Stereoscopic 3D Technologies for Accurate Depth Tasks: A Theoretical and Empirical Study. PhD thesis, "Durham University", 2011 .

[73] J. M. González-Darder and P. González-López. Diagnostic Techniques and Surgical Management of Brain Tumors, chapter Management of Brain Tumors in Eloquent Areas. InTech, 2011.

[74] P. Gunz and P. Mitteroecker. Semilandmarks: a method for quantifying curves and surfaces. Hystrix, the Italian Journal of Mammalogy, 24(1):103-109, 2013.

[75] P. Gunz, P. Mitteroecker, and F.L. Bookstein. Semilandmarks in three dimensions. In D. E. Slice, editor, Modern Morphometrics in Physical Anthropology, pages 73-98. Kluwer Academic/Plenum Publishers, New York, 2005.

[76] N. Haberland, K. Ebmeier, R. Hliscs, J. P. Grnewald, J. Silbermann, J. Steenbeck, H. Nowak, and R. Kalff. Neuronavigation in surgery of intracranial and spinal tumors. Journal of Cancer Research and Clinical Oncology, 126(9):529-541, 2000.

[77] M. Hadwiger, C. Sigg, H. Scharsach, K. Bühler, and M. Gross. RealTime Ray-Casting and Advanced Shading of Discrete Isosurfaces. Computer Graphics Forum, 24(3):303-312, 2005.

[78] N. Haj-Hosseini. Flurescence Spectroscopy for Quantitative Demarcation of Glioblastoma Using 5-Aminolevulinic Acid. $\mathrm{PhD}$ thesis, Linköping University, 2012.

[79] N. Haj-Hosseini, S. Lowndes, G. Salerud, and K. Wårdell. Blood interference in fiber-optical based fluorescence guided resection of glioma using 5-aminolevulinic acid. In Photonic Therapeutics and Diagnostics VII, 78833R. SPIE, 2011.

[80] N. Haj-Hosseini, J. C. Richter, S. Andersson-Engel, and K. Wårdell. Photobleaching behavior of protoporphyrin IX during 5-aminolevulinic acid marked glioblastoma detection. In Conference on Photonic Therapeutics and Diagnostics V, 7161. SPIE.

[81] N. Haj-Hosseini, J. C. Richter, S. Andersson-Engel, and K. Wårdell. Optical touch pointer for fluorescence guided glioblastoma resection using 5-aminolevulinic acid. Lasers in Surgery and Medicine, 42(1):9$14,2010$. 
[82] N. Haj-Hosseini, J. C. Richter, M. Hallbeck, and K. Wårdell. Low dose 5-aminolevulinic acid: Implications in spectroscopic measurements during brain tumor surgery. Photodiagnosis and Photodynamic Therapy, 12(2):209-214, 2015.

[83] D. Halazonetis. A faster solution to the sliding semilandmarks equation. Technical report, 2008.

[84] H. Hamidian, H. Soltanian-Zadeh, R. Faraji-Dana, and M. Gity. Estimating Brain Deformation During Surgery Using Finite Element Method: Optimization and Comparison of Two Linear Models. Journal of Signal Processing Systems, 55(1):157-167, 2008.

[85] T. Hartkens, D.L.G. Hill, A.D. Castellano-Smith, D.J. Hawkes, C.R. Maurer, A.J. Martin, W.A. Hall, H. Liu, and C.L. Truwit. Measurement and analysis of brain deformation during neurosurgery. IEEE Transactions on Medical Imaging, 22(1):82-92, 2003.

[86] N. Holliman. 3D Display Systems. Technical report, Durham University, 2005.

[87] H. Hori, G. Moretti, A. Rebora, and F. Crovato. The Thickness of Human Scalp: Normal and Bald. Journal of Investigative Dermatology, 58(6):396-399, 1972.

[88] T. Hosoda, H. Takeuchi, N. Hashimoto, R. Kitai, T. Kodera H. Arishima and, Y. Higashino, K. Sato, and K. Kikuta. Usefulness of Intraoperative Computed Tomography in Surgery for Low-Grade Gliomas: a Comparative Study Between Two Series Without and With Intraoperative Computed Tomography. Neurologia medico-chirurgica, 51(7):490-495, 2011.

[89] Y. Hu, E. Rijkhorst, R. Manber, D.Hawkes, and D. Barratt. Deformable Vessel-Based Registration Using Landmark-Guided Coherent Point Drift. Medical Imaging and Augmented Reality, Lecture Notes in Computer Science, 6326:60-69, 2012.

[90] R. Hubbold, D. Hancock, and C. Moore. Stereoscopic volume rendering. Proc. Visualization in Scientific Computing '98, 6(3):105-115, 1998.

[91] G. Hubona, P. Wheeler, G. Shirah, and M. Brandt. The relative contributions of stereo, lighting and background scenes in promoting 3d depth visualization. ACM Transaction on Computer-Human Interaction, 6(3):214-242, 1999.

[92] M. A. Ilias, J. Richter, F. Westermark, M. Brantmark, S. AnderssonEngels, and Karin Wårdell. Evaluation of a fiber-optic fluorescence spectroscopy system to assist neurosurgical tumor resections. In Novel 
Optical Instrumentation for Biomedical Applications III, 66310W. SPIE, 2007.

[93] V. Interrante, H. Fuchs, and S. M. Pizer. Conveying the 3D Shape of Smoothly Curving Transparent Surfaces via Texture. IEEE Transactions on Visualization and Computer Graphics, 3(2):98-117, April/ June 1997.

[94] F. E. Ives. A novel stereogram. Journal of the Franklin Institute, 153:51-52, 1902.

[95] Y. Sohma T. Saito T. Kin H. Oyama H N. Saito N J. Jiang, Y. Nakajima. Marker-less tracking of brain surface deformations by non-rigid registration integrating surface and vessel/sulci features. International Journal of Computer Assisted Radiology and Surgery, 2016.

[96] S. Ji, X. Fan, A. Hartov, D. W. Roberts, and K. D. Paulsen. An Integrated, Model-Based Neurosurgical Guidance System . In Medical Imaging 2010: Visualization, Image-Guided Procedures, and Modeling.

[97] S. Ji, X. Fan, D. W. Roberts, A. Hartov, and K. D. Paulsen. Cortical surface shift estimation using stereovision and optical flow motion tracking via projection image registration. Medical Image Analysis, 18(7):1169-1183, 2014.

[98] S. Ji, X. Fan, D. W. Roberts, and K. D. Paulsen. Cortical Surface Strain Estimation Using Stereovision. In Medical Image Computing and Computer-Assisted Intervention - MICCAI 2011, Lecture Notes in Computer Science, 6891, pages 412-419, 2011.

[99] S. Ji, X. Fan, D. W. Roberts, and K. D. Paulsen. Efficient stereo image geometrical reconstruction at arbitrary camera settings from a single calibration. In Medical Image Computing and Computer-Assisted Intervention - MICCAI 2014, Lecture Notes in Computer Science, 8673, pages 440-447, 2014.

[100] S. Ji, Z. Wu, A. Hartov, D. W. Roberts, and K. D. Paulsen. Mutualinformation-based image to patient re-registration using intraoperative ultrasound in image-guided neurosurgery. Medical Physics, 35(10):4612-4624, 2008.

[101] F. A. Jolesz. 1996 RSNA Eugene P. Pendergrass New Horizons Lecture. Image-guided procedures and the operating room of the future. Radiology, 204(3):601-612, 1997.

[102] F. A. Jolesz. Interventional and intraoperative MRI: a general overview of the field. Journal of Magnetic Resonance Imaging, 8(1):37, 1998. 
[103] F. A. Jolesz and F. Shtern. The operating room of the future. Report of the national cancer institute workshop. Imaging-guided stereotactic tumor diagnosis and treatment. Investigative Radiology, 27(4):326328, 1992.

[104] H. Jorke and M. Fritz. Infitec-A new stereoscopic visualization tool by wavelength multiplexing imaging. In Proc. Electronic Displays, 2003.

[105] C. R. Maurer Jr., J. J. McCrory, and J. M. Fitzpatrick. Estimation of accuracy in localizing externally attached markers in multimodal volume head images. In Medical Imaging 1993: Image Processing, Proc. SPIE 1898, pages 43-54. Society of Photo-Optical Instrumentation Engineers, 1993.

[106] C. R. Maurer CR Jr., J. M. Fitzpatrick, M. Y. Wang, R. L. Galloway Jr., R. J. Maciunas, and G. S. Allen. Registration of Head Volume Images Using Implantable Fiducial Markers. IEEE Transactions on Medical Imaging, 16(4):447-462, 1997.

[107] A. Kaufman, R. Yagel, and D. Cohen. Intermixing Surface and Volume Rendering. In K. H. Hoehne, H. Fuchs, and S. M. Pizer, editors, 3D Imaging in Medicine: Algorithms, Systems and Applications, pages 217-228. Springer-Verlag, 1990.

[108] M. Kersten, J. Stewart, N. Troje, and R. Ellis. Enhancing depth perception in translucent volumes. IEEE Transactions on Visualization and Computer Graphics, 12(5):1117-1124, 2006.

[109] M. Kersten-Oertel, S. J. Chen, and D. L. Collins. An Evaluation of Depth Enhancing Perceptual Cues for Vascular Volume Visualization in Neurosurgery. IEEE Transactions on Visualization and Computer Graphics, 20(3):391-403, 2014.

[110] M. Kersten-Oertel, I. J. Gerard, S. Drouin, K. Mok, D. Sirhan, D. S. Sinclair, and D. L. Collins. Augmented Reality for Specific Neurovascular Tasks. In Augmented Environments for Computer-Assisted Interventions - AE-CAI 2015, Lecture Notes in Computer Science, 9365, pages $92-103,2015$.

[111] M. Kersten-Oertel, I. J. Gerard, S. Drouin, K. Mok, D. Sirhan, D. S. Sinclair, and D. L. Collins. Augmented reality in neurovascular surgery: feasibility and first uses in the operating room. International Journal Of Computer Assisted Radiology and Surgery, 10(11):1823$1836,2015$.

[112] S. Kirkpatrick, Jr. C. D. Gelatt, and M. P. Vecchi. Optimization by Simulated Annealing. Science, 220(4598):671-680, 1983. 
[113] J. Konrad and M. Halle. 3-D displays and signal processing.

[114] A. N. Kumar, M. I. Miga, T. S. Pheiffer, L. B. Chambless, R. C. Thompson, and B. M. Dawant. Automatic tracking of intraoperative brain surface displacements in brain tumor surgery. In Engineering in Medicine and Biology Society (EMBC), 2014, 36th Annual International Conference of the IEEE, pages 1509-1512, 2014.

[115] A. N. Kumar, M. I. Miga, T. S. Pheiffer, L. B. Chambless, R. C. Thompson, and B. M. Dawant. Persistent and automatic intraoperative 3D digitization of surfaces under dynamic magnifications of an operating microscope. Stereotactic Functional Neurosurgery, 19(1):30$45,2015$.

[116] A. N. Kumar, T. S. Pheiffer, A. L. Simpson, R. C. Thompson, M. I. Miga, and B. M. Dawant. Phantom-based comparison of the accuracy of point clouds extracted from stereo cameras and laser range scanner. In Medical Imaging 2013: Image-Guided Procedures, Robotic Interventions, and Modeling, volume 8671. SPIE, 2013.

[117] R. P. Kumar, F. Albregtsen, M. Reimers, , T. Lang $\varnothing$, B. Edwin, and O. J. Elle. 3D multiscale vessel enhancement based centerline extraction of blood vessels. In Medical Imaging 2013: Image Processing, volume 8669. SPIE, 2013.

[118] R. P. Kumar, F. Albregtsen, M. Reimers, B. Edwin, T. Lang $\varnothing$, and O. J. Elle. Three-Dimensional Blood Vessel Segmentation and Centerline Extraction based on Two-Dimensional Cross-Section Analysis. Annals of Biomedical Engineering, 43(5):1223-1234, 2015.

[119] T. Kuroiwa, Y. Kajimoto, and T. Ohta. Development and clinical application of near-infrared surgical microscope: preliminary report. Minimally Invasive Neurosurgery, 44(4):240-242, 2001.

[120] M. Lacroix, D. Abi-Said, D. R. Fourney, Z. L. Gokaslan, W. Shi, F. DeMonte, F. F. Lang, I. E. McCutcheon, S. J. Hassenbusch, E. Holland, K. Hess, C. Michael, D. Miller, and R. Sawaya. A multivariate analysis of 416 patients with glioblastoma multiforme: prognosis, extent of resection, and survival. Journal of Neurosurgery, 95(2):190-198, 2001.

[121] R.J. Lapeer and R.W. Prager. 3D shape recovery of a newborn skull using thin-plate splines. Computerized Medical Imaging and Graphics, 24:193-204, 2000.

[122] R.J. Lapeer and R.W. Prager. An optimised radial basis function algorithm for fast non-rigid registration of medical images. Computers in Biology and Medicine, 40(1):1-7, 2010. 
[123] A. Lauric, R. S. Heller, S. Schimansky, and A. M. Malek. Benefit of Cone-Beam CT Angiography in Visualizing AneurysmShape and Identification of Exact Rupture Site. Journal of Neuroimaging, 25(1):5661, 2015.

[124] T. C. Lee, R. L. Kashyap, and C. N. Chu. Building Skeleton Models via 3-D Medial Surface Axis Thinning Algorithms. GVGIP: Graphical Models Image Processing, 56(6):462-478, 1994.

[125] M. Levoy. Display of Surfaces from Volume Data. IEEE Computer Graphics and Applications, 8(3):29-37, 1988.

[126] M. Levoy. A Hybrid Ray-Tracer for Rendering Polygon and Volume Data. IEEE Computer Graphics and Applications, 10(3):33-40, 1990.

[127] Y. Li, R. Rey-Dios, D. W. Roberts, and P.A. Valdés Pand A.A. Cohen-Gadol. Intraoperative fluorescence-guided resection of highgrade gliomas: a comparison of the present techniques and evolution of future strategies. World Neurosurgery, 82:175-185, 2014.

[128] W. C. Lin, S. A. Toms, E.D. Jansen, and A. Mahadevan-Jansen. In vivo brain tumor demarcation using optical spectroscopy. Photochemistry and Photobiology, 73(4):396-402, 2001.

[129] W. C. Lin, S. A. Toms, M. Motamedi, E.D. Jansen, and A. Mahadevan-Jansen. Brain tumor demarcation using optical spectroscopy; an in vitro study. Journal of Biomedical Optics, 5(2):214220,2000 .

[130] F. Lindseth, T. Langø, T. Selbekk, R. Hansen, I. Reinertsen, C. Askeland, O. Solheim, G. Unsgård, R. Mårvik, and T. A. N. Hernes. Advancements and Breakthroughs in Ultrasound Imaging, chapter Ultrasound-Based Guidance and Therapy. InTech, 2013.

[131] L. Linker. 3D with No Glasses. DV: Digital Video, 2004.

[132] Y. Liu, C. Yao, L. Zhou, and N. Chrisochoides. A point based nonrigid registration for tumor resection using iMRI. In IEEE International Symposium on Biomedical Imaging: From Nano to Macro, pages 1217-1220. IEEE, 2010.

[133] D. N. Louis, H. Ohgaki, O. D. Wiestler, W. K. Cavenee, P. C. Burger, A. Jouvet, B. W. Scheithauer, and P. Kleihues. The 2007 WHO Classification of Tumours of the Central Nervous System. Acta Neuropathologica, 114(2):97-109, 2007.

[134] K. E. Lunn, A. Hartov, F. E. Kennedy, M. I. Miga, D. W. Roberts, L. A. Platenik, and K. D. Paulsen. 3D ultrasound as sparse data for intraoperative brain deformation model. In Medical Imaging 2001: Ultrasonic Imaging and Signal Processing, volume 326. SPIE, 2001. 
[135] J. B. A. Maintz and M. A. Viergever. A survey of medical image registration. Medical Image Analysis, 2(1):1-36, 1998.

[136] F. M. M. Marreiros, S. Rossitti, T. Gustafsson, P. Carleberg, and Ö. Smedby. Multi-view 3D vessel tracking using near-infrared cameras. Int. J CARS, (Suppl. 1), Image Processing and Visualization, 2013.

[137] C. R. Maurer, Jr., F. Sauer, B. Hu, B. Bascle, B. Geiger, F. Wenzel, T. Rohlfing, C. M. Brown, R. S. Bakos, R. J. Maciunas, and A. Banihashemi. Augmented Reality Visualization of Brain Structures with Stereo and Kinetic Depth Cues: System Description and Initial Evaluation with Head Phantom. In Medical Imaging 2001: Visualization, Display, and Image-Guided Procedures, Proc. SPIE 4319, pages 445456. Society of Photo-Optical Instrumentation Engineers, 2001.

[138] L. Mercier, V. Fonov, C. Haegelen, R. F. Del Maestro, K. Petrecca, and D. L. Collins. Comparing two approaches to rigid registration of three-dimensional ultrasound and magnetic resonance images for neurosurgery. International Journal of Computer Assisted Radiology and Surgery, 7(1):125-136, 2012.

[139] M. I. Miga, J. M. Fitzpatrick, R. L. Galloway, and K. D. Paulsen. Incorporation of surface-based deformations for updating images intraoperatively. In Medical Imaging 2001: Visualization, Display, ImageGuided Procedures, volume 169. SPIE, 2001.

[140] M. I. Miga, T. K. Sinha, D. M. Cash, R. L. Galloway, and R. J. Weil. Cortical Surface Registration for Image-Guided Neurosurgery Using Laser-Range Scanning. IEEE Transactions on Medical Imaging, 22(8):973-985, 2003.

[141] M. I. Miga, K. Sun, I. Chen, L. W. Clements, T. S. Pheiffer, and A. L. Simpson andR. C. Thompson. Clinical evaluation of a modelupdated image-guidance approachto brain shift compensation: experience in 16 cases. International Journal of Computer Assisted Radiology and Surgery, pages 1-8.

[142] J. M. K. Mislow, A. J. Golby, and P. M. Black. Origins of Intraoperative Mri. Neurosurgery Clinics of North America, 20(2):137-146, 2009 .

[143] A. Mohammadi, A. Ahmadian, A. D. Azar, A. D. Sheykh, F. Amiri, and J. Alirezaie. Estimation of intraoperative brain shift by combination of stereovision and doppler ultrasound: phantom and animal model study. International Journal of Computer Assisted Radiology and Surgery, 10:1753-1764, 2015.

[144] R. Moreno and Ö. Smedby. Gradient-based enhancement of tubular structures in medical images. Medical Image Analysis, 26(1):19-29, 2015 . 
[145] R. Moreno and Ö. Smedby. Vesselness estimation through higher-order orientation tensors. In IEEE International Symposium on Biomedical Imaging, pages 1139-1142. IEEE, 2016.

[146] A. Myronenko and X. Song. Point-Set Registration: Coherent Point Drift. IEEE Transactions on Pattern Analysis and Machine Intelligence, 32(12):2262-2275, 2010.

[147] A. Nabavi, P.M. Black, D.T. Gering, Westin C.F., V. Mehta, R.S. Jr. Pergolizzi, M. Ferrant, S.K. Warfield, N. Hata, R.B. Schwartz, W.M. 2rd Wells, R. Kikinis, and F.A. Jolesz. Serial intraoperative magnetic resonance imaging of brain shift. Neurosurgery, 48(4):787-798, 2001.

[148] F. Maes nad D. Vandermeulen and P. Suetens. Medical Image Registration Using Mutual Information. Proccedings of the IEEE, 91(10):1699-1722.

[149] S. Nakajima, H. Atsumi, R. Kikinis, T. Moriarty, D. C. Metcalf, A. F., and P. McL. Black. Use of Cortical Surface Vessel Registration for Image-guided Neurosurgery. Neurosurgery, 40(6):1201-1210, 1997.

[150] K. Nakayama, S. Shimojo, and V. S. Ramachandran. Transparency: relation to depth, subjective contours, luminance, and neon color spreading. Perception, 19:497-513, 1990.

[151] A. Nealen, M. Müller, R. Keiser, E. Boxerman, and M. Carlson. Physically Based Deformable Models in Computer Graphics. In Eurographics 2005 State of The Art Report, Eurographics 2005, 2005.

[152] C. Nimsky, O. Ganslandt, S. Cerny, P. Hastreiter, G. Greiner, and R. Fahlbusch. Quantification of, visualization of, and compensation for brain shift using intraoperative magnetic resonance imaging. Neurosurgery, 47(5):1070-1079, 2000.

[153] Nvidia. 3D Vision, 2012.

[154] F. P. Oliveira and J. M. Tavares. Medical image registration: a review. Computer Methods in Biomechanics and Biomedical Engineering, 17(2):73-93, 2014.

[155] R. C. Orth, M. J. Wallace, and M. D. Kuo. C-arm Cone-beam CT: General Principles and Technical Considerations for Use in Interventional Radiology. Journal of Vascular and Interventional Radiology, 19(6):814-820, 2008.

[156] J. Owczarczyk and B. Owczarczyk. Evaluation of true 3D display systems for visualizing medical volume data. The Visual Computer, 6(4):219-226, 1990. 
[157] S. Parker, P. Shirley, Y. Livnat, C. Hansen, and P-P. Sloan. Interactive ray tracing for isosurface rendering. In Visualization '98, pages 233238. ACM, 1998.

[158] P. Paul, X. Morandi, and P. Jannin. A Surface Registration Method for Quantification of Intraoperative Brain Deformations in ImageGuided Neurosurgery. IEEE Transactions on Information Technology in Biomedicine, 13(6):976-983, 2009.

[159] T. S. Pheiffer, A. L. Simpson, B. Lennon, R. C. Thompson, and M. I. Miga. Design and evaluation of an optically-tracked single-CCD laser range scanner. Medical Physics, 39(2):636-642, 2012.

[160] F. Pomerleau, F. Colas, and R. Siegwart. A review of point cloud registration algorithms for mobile robotics. Foundations and Trends in Robotics, 4(1):1-104, 2015.

[161] R. Pottier, B. Krammer, R. Baumgartner, and H. Stepp. Photodynamic Therapy with ALA : A Clinical Handbook. Royal Society of Chemistry, 2006.

[162] B. Preim and D. Bartz. Visualization in Medicine: Algorithms, Systems and Applications. The Morgan Kaufmann Series in Computer Graphics, 2007.

[163] B. Preim and C. P. Botha. Visual Computing for Medicine: Algorithms, Systems and Applications. The Morgan Kaufmann Series in Computer Graphics, 2 edition, 2013.

[164] A. Raabe, J. Beck, R. Gerlach, M. Zimmermann, and V. Seifert. Nearinfrared indocyanine green video angiography: a new method for intraoperative assessment of vascular flow. Neurosurgery, 52(2):132-139, 2003.

[165] A. Raabe, P. Nakaji, J. Beck, L.J. Kim, F.P. Hsu, J. D. Kamerman, V. Seifert, and R. F. Spetzler. Prospective evaluation of surgical microscope-integrated intraoperative near-infrared indocyanine green videoangiography during aneurysm surgery. Journal of Neurosurgery, 103(6):982-989, 2005.

[166] I. Reinertsen, M. Descoteaux, K. Siddiqi, and D. L. Collins. Validation of vessel-based registration for correction of brain shift. Medical Image Analysis, 11(4):374-388, 2007.

[167] I. Reinertsen, F. Lindseth, C. Askeland, Daniel H. Iversen, and G. Unsgård. Intra-operative correction of brain-shift. Acta Neurochirurgica, 156(7):1301-310, 2014. 
[168] I. Reinertsen, F. Lindseth, G. Unsgård, and D. L. Collins. Clinical validation of vessel-based registration for correction of brain-shift. Medical Image Analysis, 11(6):673-684, 2007.

[169] J. C. Richter, N. Haj-Hosseini, S. Andersson-Engel, and K. Wårdell. Fluorescence Spectroscopy Measurements in Ultrasonic Navigated Resection of Malignant Brain Tumors. Lasers in Surgery and Medicine, 43(1):8-14, 2011.

[170] D. W. Roberts, A. Hartov, F. E. Kennedy, M. I. Miga, and K. D. Paulsen. Intraoperative Brain Shift and Deformation: A Quantitative Analysis of Cortical Displacement in 28 Cases. Neurosurgery, 43(4):749-760, 1998.

[171] R. S. Rowland. Fast Registration of Medical Imaging Data Using Optimised Radial Basis Functions :PhD Dissertation. PhD thesis, University of East Anglia, 2007.

[172] S. Rusinkiewicz and M. Levoy. Efficient Variants of the ICP Algorithm. In 3-D Digital Imaging and Modeling, pages 145-152. IEEE, 2001.

[173] Y. Sato, S. Nakajima, N. Shiraga, H. Atsumi, S. Yoshida, T. Koller, G. Gerig, and R. Kikinis. Three-dimensional multi-scale line filter for segmentation and visualization of curvilinear structures in medical images. Medical Image Analysis, 2(2):143-168, 1998.

[174] P. H. Schönemann. A generalized solution of the orthogonal Procrustes problem. Psychometrika, 31:1-10, 1994.

[175] J. Sethian. Level Set Methods and Fast Marching Methods. Cambridge University Press, 2 edition, 1999.

[176] R. R Shamir, L. Joskowicz, and Y. Shoshan. Optimal landmarks selection and fiducial marker placement for minimal target registration error in image-guided neurosurgery. In Medical Imaging 2009: Visualization, Image-Guided Procedures, and Modeling, volume 7261. SPIE, 2009.

[177] R. R Shamir, L. Joskowicz, and Y. Shoshan. Fiducial Optimization for Minimal Target Registration Error in Image-Guided Neurosurgery. IEEE Transactions on Medical Imaging, 31(3):725-737, 2012.

[178] T. Sielhorst, M. Feuerstein, and N. Navab. Advanced Medical Displays: A Literature Review of Augmented Reality. Journal of Display Technology, 4(4):451-467, 2008.

[179] C. Sigg. Representation and Rendering of Implicit Surfaces. PhD thesis, ETH Zurich, 2006. 
[180] T. K. Sinha, B. M. Dawant, V. Duay, D. M. Cash, R. J. Weil, R. C. Thompson, K. D. Weaver, and M. I. Miga. A Method to Track Cortical Surface Deformations Using a Laser Range Scanner. IEEE Transactions on Medical Imaging, 24(6):767-781, 2005.

[181] T. K. Sinha, V. Duay, B. M. Dawant, and M. I. Miga. Cortical Surface Registration Using Texture Mapped Point Clouds and Mutual Information. In Medical Image Computing and Computer-Assisted Intervention - MICCAI 2002, Lecture Notes in Computer Science, 2489, pages 533-540, 2002.

[182] T. K. Sinha, V. Duay, B. M. Dawant, and M. I. Miga. Cortical Shift Tracking Using a Laser Range Scanner and Deformable Registration Methods. In Medical Image Computing and Computer-Assisted Intervention - MICCAI 2003, Lecture Notes in Computer Science, 2879, pages 166-174, 2003.

[183] T. K. Sinha, V. Duay, B. M. Dawant, and M. I. Miga. Laser range scanning for cortical surface characterization during neurosurgery. In Medical Imaging 2003: Visualization, Image-Guided Procedures, and Display, volume 5029, pages 98-107. SPIE, 2003.

[184] A. Sironi, E. Turetken, V. Lepetit, and Pascal Fua. Multiscale Centerline Detection. IEEE Transactions on Pattern Analysis and Machine Intelligence, 38(7):1327-1341, 2016.

[185] D. Slice. Modern Morphometrics in Physical Anthropology. Kluwer Academic/Plenum Publishers, 2005.

[186] E. Smistad, T. L. Falch, M. Bozorgi, A. C. Elster, and F. Lindseth. Medical image segmentation on GPUs - A comprehensive review. Medical Image Analysis, 20(1):1-18, 2015.

[187] A. Soltanipour, S.Sadri, H. Rabbani, M. Akhlaghi, and A. DoostHosseini. Vessel centerlines extraction from fundus fluorescein angiogram based on hessian analysis of directional curvelet subbands. In IEEE International Conference on Acoustics, Speech and Signal Processing (ICASSP), pages 1070-1074, 2013.

[188] W. Stummer, A. Novotny, H. Stepp, C. Goetz, K. Bise, and H. J. Reulen. Intraoperative detection of malignant gliomas by 5aminolevulinic acid-induced porphyrin fluorescence. Neurosurgery, 42(3):518-526, 1998.

[189] W. Stummer, A. Novotny, H. Stepp, C. Goetz, K. Bise, and H. J. Reulen. Fluorescence-guided resection of glioblastoma multiforme by using 5-aminolevulinic acid-induced porphyrins: a prospective study in 52 consecutive patients. Journal of Neurosurgery, 93(6):1003-1013, 2000 . 
[190] W. Stummer, H. J. Reulen, T. Meinel, U. Pichlmeier, W. Schumacher, J. C. Tonn, V. Rohde, F. Oppel, B. Turowski, C. Woiciechowsky, K. Franz, and T. Pietsch. Fluorescence-guided surgery with 5aminolevulinic acid for resection of malignant glioma: a randomised controlled multicentre phase iii trial. Lancet Oncology, 7(5):392-401, 2006.

[191] W. Stummer, H. J. Reulen, T. Meinel, U. Pichlmeier, W. Schumacher, J. C. Tonn, V. Rohde, F. Oppel, B. Turowski, C. Woiciechowsky, K. Franz, and T. Pietsch. Extent of resection and survival in glioblastoma multiforme: identification of and adjustment for bias. Neurosurgery, 62(3):564-576, 2008.

[192] H. Sun, H. Farid, D. W. Roberts, K. Rick, A. Hartov, and K. D. Paulsen. Estimating Cortical Surface Motion Using Stereopsis for Brain Deformation Models. In Medical Image Computing and Computer-Assisted Intervention - MICCAI 2013, Lecture Notes in Computer Science, 2878, pages 794-801, 2003.

[193] H. Sun, H. Farid, D. W. Roberts, K. Rick, A. Hartov, and K. D. Paulsen. A noncontacting 3-D digitizer for use in image-guided neurosurgery. Stereotactic Functional Neurosurgery, 80:120-124, 2003.

[194] H. Sun, K. E. Lunn, H. Farid, Z. Wu, D. W. Roberts, A. Hartov, and K. D. Paulsen. Stereopsis-guided brain shift compensation. IEEE Transactions on Medical Imaging, 24(8):1039-1052, 2005.

[195] K. Sun, T. S. Pheiffer, A. L. Simpson, J. A. Weis, R. C. Thompson, and M. I. Miga. Near Real-Time Computer Assisted Surgery for Brain Shift Correction Using Biomechanical Models. IEEE Journal of Translational Engineering in Health and Medicine, 2, 2014.

[196] Y. Takagi, K. Sawamura, and N. Hashimoto. Intraoperative Nearinfrared Indocyanine Green Videoangiography Performed with a Surgical Microscope - Applications in Cerebrovascular Surgery. European Neurological Review, 3(1):66-68, 2008.

[197] J. T. Todd. The visual perception of 3D shape. TRENDS in Cognitive Sciences, 8(3):115-121, 2004.

[198] S. Tourancheau, M. Sjöström, R. Olsson, A. Persson, J. Rudling, T. Ericson, and B. Norén. Subjective evaluation of user experience in interactive 3D visualization in a medical context. Proc. SPIE, 8318(831814):219-226, 2012.

[199] I. Tsirlin, R. S. Allison, and L. M. Wilcox. Stereoscopic transparency: Constraints on the perception of multiple surfaces. Journal of Vision, 8(5):1-10, May 2008. 
[200] G. Turk and J. F. O'Brien. Modelling with implicit surfaces that interpolate. ACM Transactions on Graphics, 21(4):855-873, 2002.

[201] H. Tuy and L. Tuy. Direct 2D display of 3D objects. IEEE Computer Graphics and Applications, 4:29-33, 1984.

[202] S. Utsuki, H. Oka, S. Sato, S. Suzuki, S. Shimizu, S. Tanaka, and K. Fujii. Possibility of using laser spectroscopy for the intraoperative detection of nonfluorescing brain tumors and the boundaries of brain tumor infiltrates. Journal of Neurosurgery, 104(4):618-620, 2006.

[203] A. L. Vahrmeijer, M. Hutteman, J. R. van der Vorst, C. J. H. van de Velde, and J. V. Frangioni. Image-guided cancer surgery using nearinfrared fluorescence. Nature Reviews Clinical Oncology, 10:507-518, 2013.

[204] P. A. Valdés, A. Kim, F. Leblond, O. M. Conde, B. T. Harris, K. D. Paulsen, B. C. Wilson, and D. W. Roberts. Combined fluorescence and reflectance spectroscopy for in vivo quantification of cancer biomarkers in low- and high-grade glioma surgery. Journal of Biomedical Optics, 16(11), 2011.

[205] P. A. Valdés, F. Leblond, A. Kim, B. T. Harris, B. C. Wilson, X. Fan, T. D. Tosteson, A. Hartov, S. Ji, K. Erkmen, N. E. Simmons, K. D. Paulsen, and D. W. Roberts. Quantitative fluorescence in intracranial tumor: implications for ALA-induced PpIX as an intraoperative biomarker. Journal of Neurosurgery, 115(1):11-17, 2011.

[206] M.A. Viergever, J.B.A. Maintz, W.J. Niessen, H.J. Noordmans, J.P.W. Pluim, and R. Stokking andK.L. Vincken. Registration, segmentation, and visualization of multimodal brain images. Computerized Medical Imaging ans Graphics, 25:147-151, 2001.

[207] L. M. Vigneron, L. Noels, S. K. Warfield, J. G. Verly, and P. A. Robe. Serial FEM/XFEM-Based Update of Preoperative Brain Images Using Intraoperative MRI. International Journal of Biomedical Imaging, 2012(2):1-17, 2012.

[208] R. Vivanti, L. Joskowicz, O. Sadowsky, and M. Shoham. Robust surface brain tissue deformation tracking in open craniotomies. International Journal of Computer Assisted Radiology and Surgery, (Suppl. 1), Image Processing and Visualization, 2013.

[209] F. Volonté, N.C. Buchs, F. Pugin, J. Spaltenstein, M. Jung, O. Ratib, and P. Morel. Stereoscopic augmented reality for da Vinci ${ }^{\mathrm{TM}}$ robotic biliary surgery. International Journal of Surgical Case Reports, $4(4): 365-367,2013$. 
[210] O. Škrinjar and J. Duncan. Stereo-guided volumetric deformation recovery. In IEEE International Symposium on Biomedical Imaging 2002, pages 863-866. IEEE, 2002.

[211] O. Škrinjar, C. Studholme, A. Nabavi, and J. Duncan. Steps Toward a Stereo-Camera-Guided Biomechanical Model for Brain Shift Compensation. In Information Processing in Medical Imaging, Lecture Notes in Computer Science, 2082, pages 183-189, 2001.

[212] C. Wang, R. Moreno, and Ö. Smedby. Vessel Segmentation Using Implicit Model-Guided Level Sets. In the 3D Cardiovascular Imaging: A MICCAI Segmentation Challenge Workshop, 2012.

[213] C. Wang and Ö. Smedby. Fully automatic brain segmentation using model-guided level sets and skeleton-based models. MIDAS Journal, 2013.

[214] Z. Wang, A. C. Bovik, H. R. Sheikh, and E. P. Simoncelli. Image quality assessment: From error visibility to structural similarity. IEEE Transactions on Image Processing, 13:600-612, 2004.

[215] S. K. Warfield, F. Talos, A. Tei, A. Bharatha, A. Nabavi, M. Ferrant, P. M. Black, F. A. Jolesz, and R. Kikinis. Real-Time Registration of Volumetric Brain MRI by Biomechanical Simulation of Deformation during Image Guided Neurosurgery. Journal of Computing and Visualization in Science, 5(2):3-11, 2002.

[216] J. R. Watson, C. F. Gainer, N. Martirosyan, J. Skoch, Jr. G. M. Lemole R. Anton, and M. Romanowskia. Augmented microscopy: real-time overlay of bright-field and near-infrared fluorescence images. Journal of Biomedical Optics, 20(10), 2015.

[217] R. Westermann and C. Rezk-Salama. Real-time volume deformations. Computer Graphics Forum, 20(3), 2001.

[218] C. Wickens and L. Thomas. Effects of CDTI display dimensionality and conflict geometry on conflict resolution performance. In Proceedings of the 13th International Symposium on Aviation Psychology, 2005.

[219] G. Widhalm. 5-Aminolevulinic Acid (5-ALA) in Brain Tumor Surgery. PhD thesis, Medical University of Vienna, 2013.

[220] A. Woods. Understanding Crosstalk in Stereoscopic Displays. ThreeDimensional Systems and Applications, 2010.

[221] H. P. Wu and S. Chang. Design of stereoscopic viewing system based on a compact mirror and dual monitor. SPIE Optical Engineering 027401, 49(2):1-6, 2010. 
[222] C. Xiao, M. Staring, Y. Wang, D. P. Shamonin, and B. C. Stoel. Multiscale bi-Gaussian filter for adjacent curvilinear structures detection with application to vasculature images. IEEE Transactions on Image Processing, 22(1):174-188, 2013.

[223] H. Xie, N. Haj-Hosseini, J. Richter, K. Wårdell, and S. AnderssonEngels. Fluorescence Spectroscopy for Guiding Malignant Brain Tumor Resection with Optical Touch Pointer. In Communications and Photonics Conference and Exhibition (ACP), 2010 Asia. IEEE, 2010.

[224] J. Yang. The thin plate spline robust point matching (TPS-RPM) algorithm: A revisit. Pattern Recognition Letters, 32(7):910-918, 2011.

[225] J. Yang, S. Ma, Q. Sun, W. Tan, M. Xu, N. Chen, and D. Zhao. Improved Hessian multiscale enhancement filter. Bio-Medical Materials and Engineering, 24(6):3267-3275, 2014.

[226] X. Yang, L. W. Clements, R. H. Conley, R. C. Thompson, B. M. Dawant, and M. I. Miga. A novel craniotomy simulation system for evaluation of stereo-pair reconstruction fidelity and tracking. In Medical Imaging 2016: Image-Guided Procedures, Robotic Interventions, and Modeling, volume 9786. SPIE, 2016.

[227] Z. Zhang. Iterative Point Matching for Registration of Free-Form Curves and Surfaces. International Journal of Computer Vision, 13(2):119-154, 1994.

[228] L. Zheng, Y. Wu, and K. Ma. Perceptually Based Depth-Ordering Enhancement for Direct Volume Rendering. IEEE Transactions on Visualization and Computer Graphics, 19(3):446-459, 2013. 



\section{Publications}

The articles associated with this thesis have been removed for copyright reasons. For more details about these see:

http://urn.kb.se/resolve?urn=urn:nbn:se:liu:diva-130791 